\title{
Dealkanative Main Group Couplings Across the peri-Gap
}

Laurence J. Taylor, Michael Bühl, Brian A. Chalmers, Matthew J. Ray, Piotr Wawrzyniak, John C. Walton, David B. Cordes, Alexandra M. Z. Slawin, J. Derek Woollins, Petr Kilian*

\section{Contents}

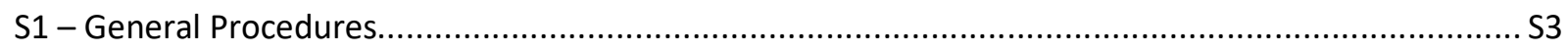

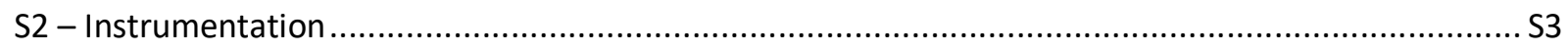

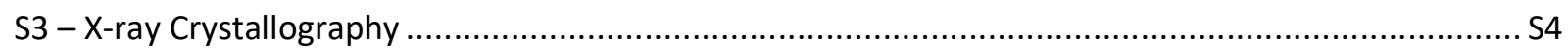

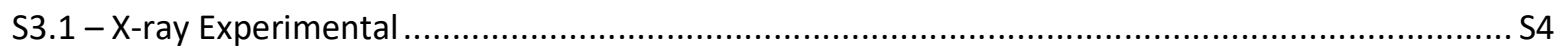

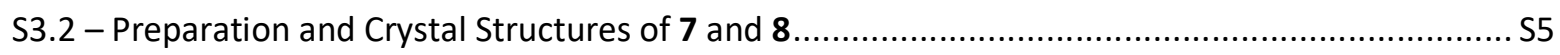

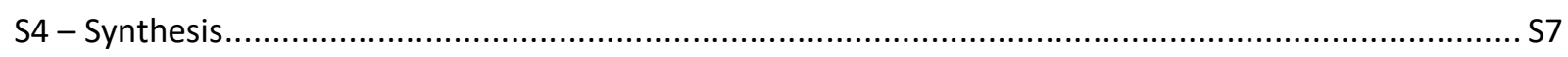

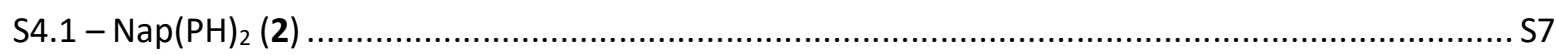

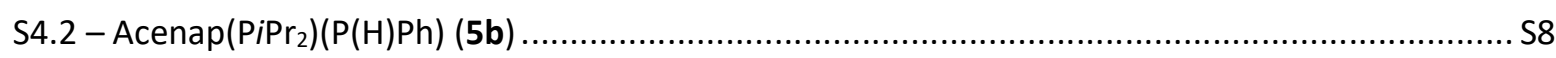

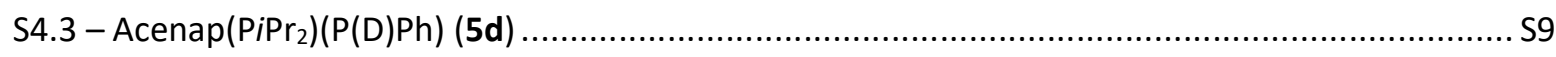

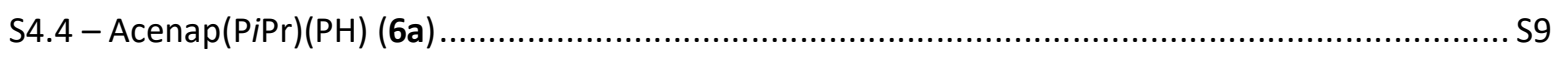

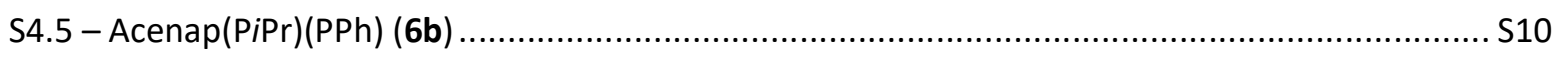

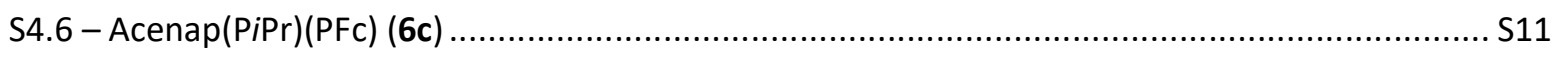

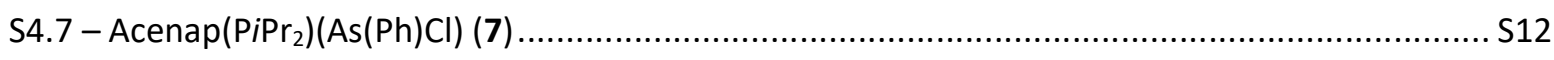

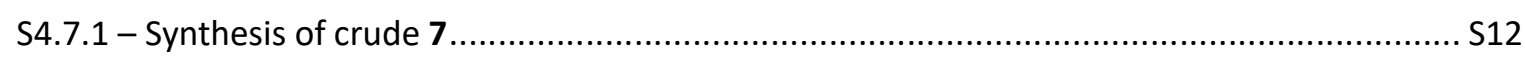

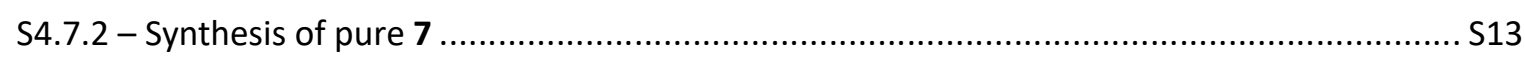

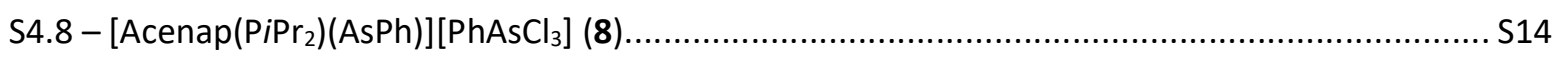

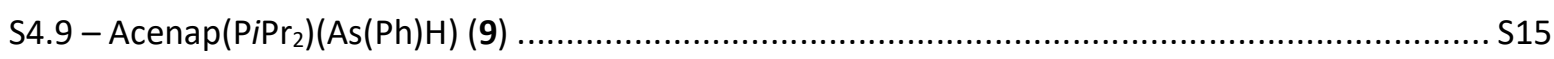

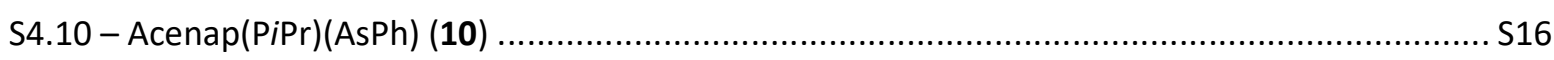

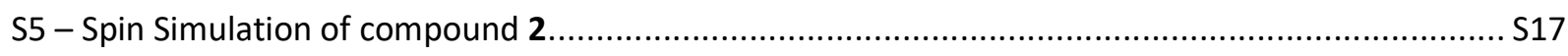

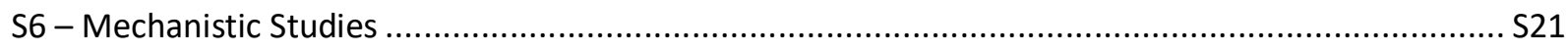

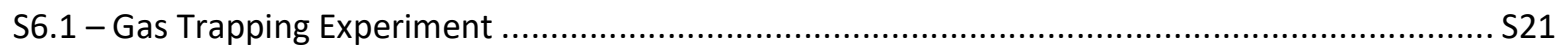

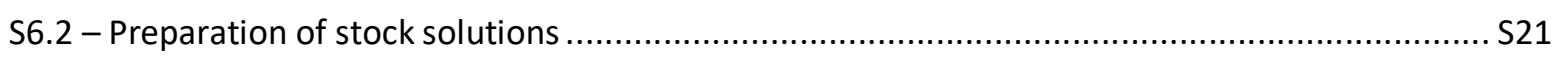

S6.3 - Methodology of Kinetic Experiments ............................................................................... 221 
S6.3.1 - General considerations

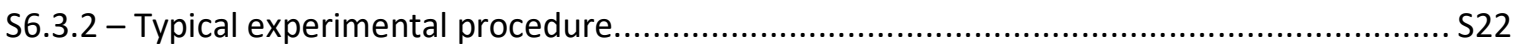

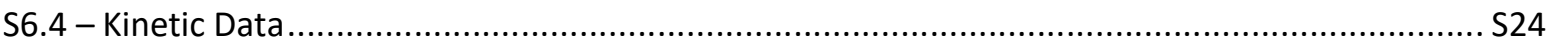

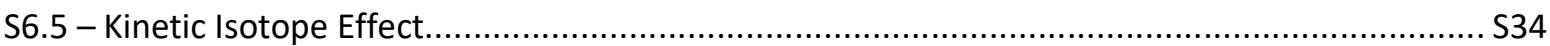

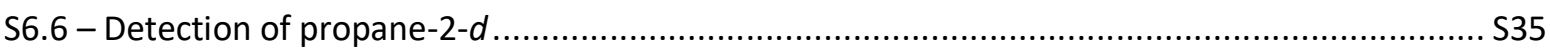

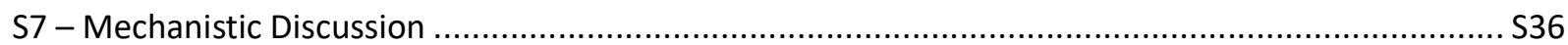

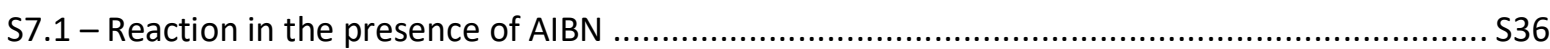

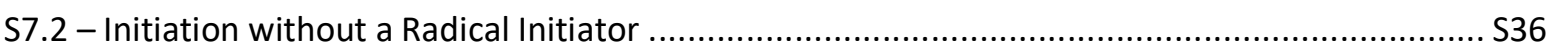

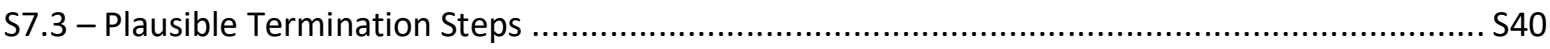

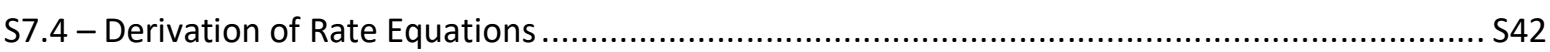

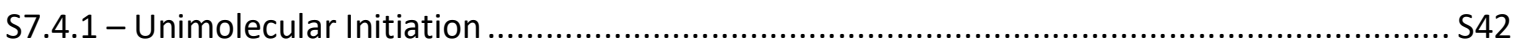

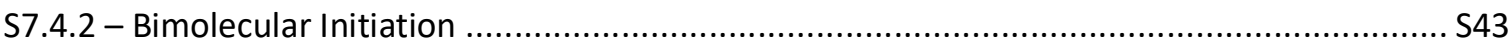

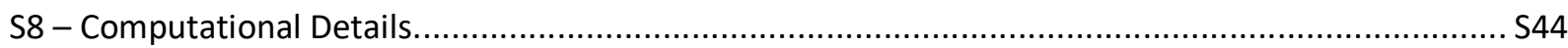

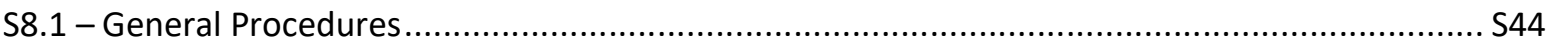

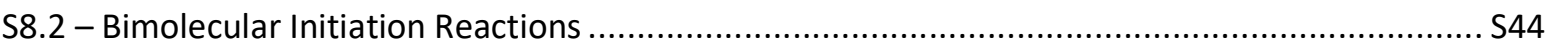

S8.3 - Cartesian Co-ordinates of Optimised Structures and Transition States.................................. S44

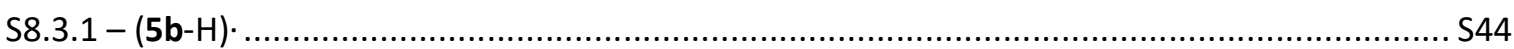

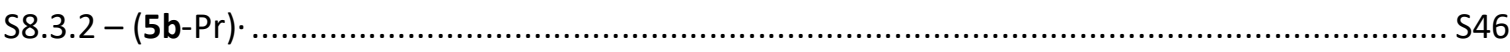

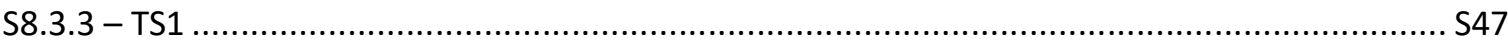

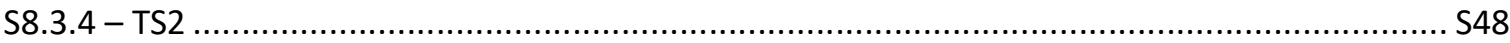

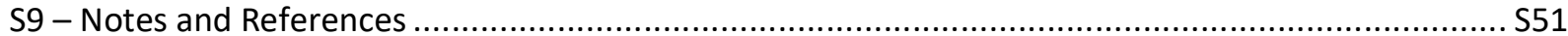




\section{S1 - General Procedures}

All experiments were carried out using standard Schlenk technique or a glove box unless otherwise stated. Solvents were dried on a MBraun solvent purification system and stored over molecular sieves prior to use. Chemicals were purchased from Sigma Aldrich, Acros Organics, Fluorochem, Alfa Aesar, Strem Chemicals Ltd., Apollo Scientific, or were taken from the laboratory inventory and were typically used without further purification. Azobisisobutyronitrile (AIBN) was recrystallised from methanol prior to use and stored at $5{ }^{\circ} \mathrm{C}$ under argon. 5-Bromo-6-diisopropylphosphinoacenaphthene, $\mathrm{Nap}\left(\mathrm{P}_{2} \mathrm{Cl}_{6}\right)$ ( $\mathrm{Nap}=$ naphthalene-1,8-diyl), [Acenap( $\left.\left.\mathrm{PiPr}_{2}\right)(\mathrm{PPh})\right][\mathrm{Cl}]$ (Acenap = Acenaphthene-5,6-diyl), and bis(phosphines) 5a and $\mathbf{5} \mathbf{c}$ were synthesised according to previously published procedures. ${ }^{1-4}$ Compound $\mathbf{5 b}$ was synthesised according to a modified version of the literature procedure, ${ }^{3}$ details of the modified procedure can be found in section S4.2. ${ }^{5}$ Where possible, new compounds were fully characterized by ${ }^{31} \mathrm{P},{ }^{31} \mathrm{P}\left\{{ }^{1} \mathrm{H}\right\},{ }^{1} \mathrm{H}$ and ${ }^{13} \mathrm{C}\left\{{ }^{1} \mathrm{H}\right\}$ NMR, including measurement of ${ }^{1} \mathrm{H}\left\{{ }^{31} \mathrm{P}\right\}, \mathrm{H}-\mathrm{H}$ DQF COSY, $\mathrm{H}-\mathrm{P} H M Q C, \mathrm{H}-\mathrm{C} H S Q \mathrm{C}$, and $\mathrm{H}-\mathrm{C}$ HMBC experiments. The NMR numbering scheme for most compounds discussed is shown in Scheme S1.

\section{S2 - Instrumentation}

All NMR spectra were recorded using a JEOL GSX Delta 270, Bruker Avance 300, Bruker Avance 400, Bruker Avance 500, or Bruker Avance III 500 spectrometer. $85 \% \mathrm{H}_{3} \mathrm{PO}_{4}$ was used as an external standard in ${ }^{31} \mathrm{P}$; ${ }^{1} \mathrm{H}$ and ${ }^{13} \mathrm{C}$ NMR were referenced to the solvent residual signal $\left(\mathrm{CDCl}_{3}: \delta \mathrm{H}=7.26, \delta \mathrm{C}=77.16 ; \mathrm{C}_{6} \mathrm{D}_{6}: \delta \mathrm{H}=\right.$ 7.16, $\delta \mathrm{C}=128.06$;). ${ }^{2} \mathrm{H}$ NMR spectra were run unlocked in xylenes (isomer mixture), with chemical shifts referenced to the residual deuterium peak of the methyl group of $p$-xylene $(\delta \mathrm{D}=2.14) .{ }^{6} \mathrm{All}$ measurements were performed at $25^{\circ} \mathrm{C}$.

EPR spectra were obtained at $9.5 \mathrm{GHz}$ with $100 \mathrm{kHz}$ modulation employing a Bruker EMX 10/12 spectrometer fitted with a rectangular ER4122 SP resonant cavity. For photolysis experiments, solutions of $5 \mathbf{b}(0.026 \mathrm{mmol})$ and di-tert-butyl peroxide $(0.054 \mathrm{mmol})$ in tert-butylbenzene $(0.25 \mathrm{~mL})$ were prepared under argon in $4 \mathrm{~mm}$ o.d. quartz tubes. Photolysis in the resonant cavity was performed by unfiltered light from a $500 \mathrm{~W}$ mercury arc lamp at temperatures in the range 250-300 K. For thermal experiments, a 52.8 $\mathrm{mM}$ solution of $\mathbf{5 b}$ in xylenes was prepared under argon in $4 \mathrm{~mm}$ o.d. quartz tubes. Measurements were carried out at temperatures in the range 300-370 K. The majority of EPR spectra were recorded with 2.0 $\mathrm{mW}$ power, $0.8 \mathrm{G}_{\mathrm{pp}}$ modulation intensity and gain of $c a .10^{6}$.

All IR and Raman spectra were obtained in the range $4000-300 \mathrm{~cm}^{-1}$ on a Perkin-Elmer System 2000 FT spectrometer. Mass spectra were acquired by the EPSRC UK National Mass Spectrometry Facility at Swansea University on a Waters Xevo G2-S, or Thermo Scientific LTQ Orbitrap XL, or by Mrs Caroline Horseburgh at the University of St Andrews on a Micromass LCT. Elemental analysis (C, H and N) was performed by Mr Stephen Boyer at London Metropolitan University. 


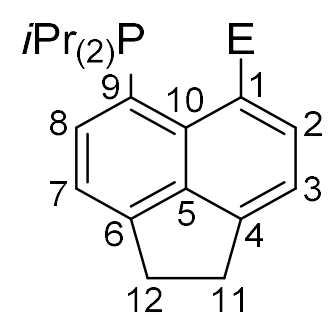

Scheme S1: NMR numbering scheme for all compounds discussed, except for $\mathrm{Nap}(\mathrm{PH})_{2}(2$, see section S5). E = P, As.

\section{S3 - X-ray Crystallography S3.1 - X-ray Experimental}

Table S1: Crystallographic data for 2, 5b, 6b, 6c, 7, 8, and 10.

\begin{tabular}{|c|c|c|c|c|c|c|c|}
\hline & 2 & $5 b$ & $6 b$ & $6 c$ & 7 & 8 & 10 \\
\hline chemical formula & $\mathrm{C}_{10} \mathrm{H}_{8} \mathrm{P}_{2}$ & $\mathrm{C}_{24} \mathrm{H}_{28} \mathrm{P}_{2}$ & $\mathrm{C}_{21} \mathrm{H}_{20} \mathrm{P}_{2}$ & $\mathrm{C}_{25} \mathrm{H}_{24} \mathrm{FeP}_{2}$ & $\mathrm{C}_{24} \mathrm{H}_{27} \mathrm{AsClP}$ & $\mathrm{C}_{30} \mathrm{H}_{32} \mathrm{As}_{2} \mathrm{Cl}_{3} \mathrm{P}$ & $\mathrm{C}_{21} \mathrm{H}_{20} \mathrm{AsP}$ \\
\hline formula weight & 190.12 & 378.43 & 334.34 & 442.26 & 456.83 & 679.76 & 378.28 \\
\hline \multirow{2}{*}{ crystal dimensions (mm) } & $0.07 \times 0.06 \times$ & $0.10 \times 0.03 x$ & $0.03 \times 0.03 x$ & $0.25 \times 0.17 \times$ & $0.20 \times 0.10$ & $0.21 \times 0.05 \times$ & $0.10 \times 0.08 \times$ \\
\hline & 0.02 & 0.03 & 0.03 & 0.16 & $\times 0.10$ & 0.03 & 0.03 \\
\hline crystal system & monoclinic & orthorombic & triclinic & triclinic & monoclinic & monoclinic & triclinic \\
\hline space group & $12 / a$ & $\mathrm{Pna}_{1}$ & $\mathrm{P}-1$ & $\mathrm{P}-1$ & $\mathrm{P} 2_{1} / \mathrm{c}$ & $\mathrm{P} 22_{1} / \mathrm{n}$ & $\mathrm{P}-1$ \\
\hline$a(\AA)$ & $14.908(6)$ & $8.234(2)$ & $9.2490(15)$ & $9.913(6)$ & $15.974(3)$ & $14.745(2)$ & $8.1289(17)$ \\
\hline$b(\AA ̊)$ & $8.201(3)$ & $41.490(11)$ & $13.144(3)$ & $10.208(5)$ & $7.6238(13)$ & $7.8199(12)$ & $11.214(2)$ \\
\hline$c(\AA)$ & $15.681(4)$ & $5.9199(16)$ & $14.882(2)$ & $12.305(7)$ & $18.084(4)$ & $25.564(4)$ & $20.239(5)$ \\
\hline$\alpha$ (deg) & 90 & 90 & $89.036(11)$ & $95.38(4)$ & 90 & 90 & $84.892(11)$ \\
\hline$B$ (deg) & $113.69(3)$ & 90 & $72.407(8)$ & $106.68(3)$ & $106.337(5)$ & $95.712(3)$ & $83.6167(10)$ \\
\hline$v$ (deg) & 90 & 90 & $87.425(11)$ & $117.00(4)$ & 90 & 90 & $70.879(8)$ \\
\hline$V\left(\AA^{3}\right)$ & $1755.6(11)$ & $2022.4(9)$ & $1722.8(5)$ & $1025.5(11)$ & $2113.5(7)$ & $2933.0(8)$ & $1729.6(6)$ \\
\hline Z & 8 & 4 & 4 & 2 & 4 & 4 & 4 \\
\hline$D_{\text {calc }}\left(\mathrm{g} \mathrm{cm}^{-3}\right)$ & 1.439 & 1.243 & 1.289 & 1.432 & 1.436 & 1.539 & 1.453 \\
\hline$\mu\left(\mathrm{cm}^{-1}\right)$ & 4.282 & 19.665 & 2.492 & 8.988 & 18.173 & 26.254 & 20.551 \\
\hline no. rflns measured (unique) & 13718(1607) & 21054(3638) & 21138(6255) & $9216(3746)$ & $16975(3877)$ & 28423(5368) & $24148(6291)$ \\
\hline $\mathrm{R}^{a}$ & 0.0445 & 0.0547 & 0.0805 & 0.0273 & 0.0199 & 0.0215 & 0.0278 \\
\hline $\mathrm{wR} 2^{b}$ & 0.0899 & 0.1354 & 0.2022 & 0.0726 & 0.0553 & 0.0577 & 0.0659 \\
\hline
\end{tabular}

Table S1 lists details of data collections and refinements. Data for compounds $\mathbf{2}$ and $\mathbf{6 b}$ were collected at $-100(1){ }^{\circ} \mathrm{C}$ on a Rigaku XtaLAB P200 diffractometer using Mo K $\alpha$ radiation $(\lambda=0.71075 \AA$ ). Data for compound $\mathbf{5 b}$ were collected at $-148(1){ }^{\circ} \mathrm{C}$ on a Rigaku XtaLAB P200 diffractometer using $\mathrm{Cu}$ Ka radiation $(\lambda=1.54187 \AA)$. Data for compound $6 \mathrm{c}$ were collected at $-100(1)^{\circ} \mathrm{C}$ on a Mercury 70 diffractometer using Mo Ka radiation $\left(\lambda=0.71075 \AA\right.$ ). . Data for compounds 7 and 8 were collected at $-180(1){ }^{\circ} \mathrm{C}$ on a Rigaku XtaLAB P100 diffractometer using Mo K $\alpha$ radiation $(\lambda=0.71075 \AA$ ). Data for compound 10 were collected at $-180(1){ }^{\circ} \mathrm{C}$ on a Rigaku XtaLAB P200 diffractometer using Mo K $\alpha$ radiation $(\lambda=0.71075 \AA$ ). Intensities were corrected for Lorentz polarization and for absorption. The structures were solved by direct methods. Refinements were done by full-matrix least-squares based on $\mathrm{F}^{2}$ using SHELXTL. ${ }^{7}$ CCDC 1566656-1566662 contain the supplementary crystallographic data for this paper. These data can be obtained free of charge from The Cambridge Crystallographic Data Centre via www.ccdc.cam.ac.uk/data_request/cif. Ellipsoids in all ORTEP plots are drawn with $50 \%$ probability. 


\section{S3.2 - Preparation and Crystal Structures of 7 and 8}

The reaction of Acenap $\left(\mathrm{PiPr}_{2}\right)(\mathrm{Li})$ (Acenap = Acenaphthene-5,6-diyl) with phenyldichloroarsine afforded 7 and $\mathbf{8}$ as shown in Scheme $\mathrm{S} 2$. Compound $\mathbf{8}$ is formed by halide abstraction from $\mathbf{7}$ by $\mathrm{PhAsCl}_{2}$. Additionally, 7 can react with another equivalent of Acenap $\left(\mathrm{PiPr}_{2}\right)(\mathrm{Li})$ to give the doubly substituted product 11, resulting in an excess of $\mathrm{PhAsCl}_{2}$ being present in the reaction mixture (Scheme S2). As a result, it was not possible to obtain pure $\mathbf{7}$ from this reaction.

It was found that a higher ratio of 7:8 was obtained when phenyldichloroarsine was added rapidly to the solution of lithiated acenaphthene (over a few minutes), rather than dropwise over a longer period. Pure 8 was obtained by reaction with 2 equivalents $\mathrm{PhAsCl}_{2}$ (see $\mathrm{Sl}$, section $\mathrm{S} 4.8$ ), and pure 7 was obtained by the reaction of 9 with chloroform (see SI, sections S4.7.2 and S4.9).

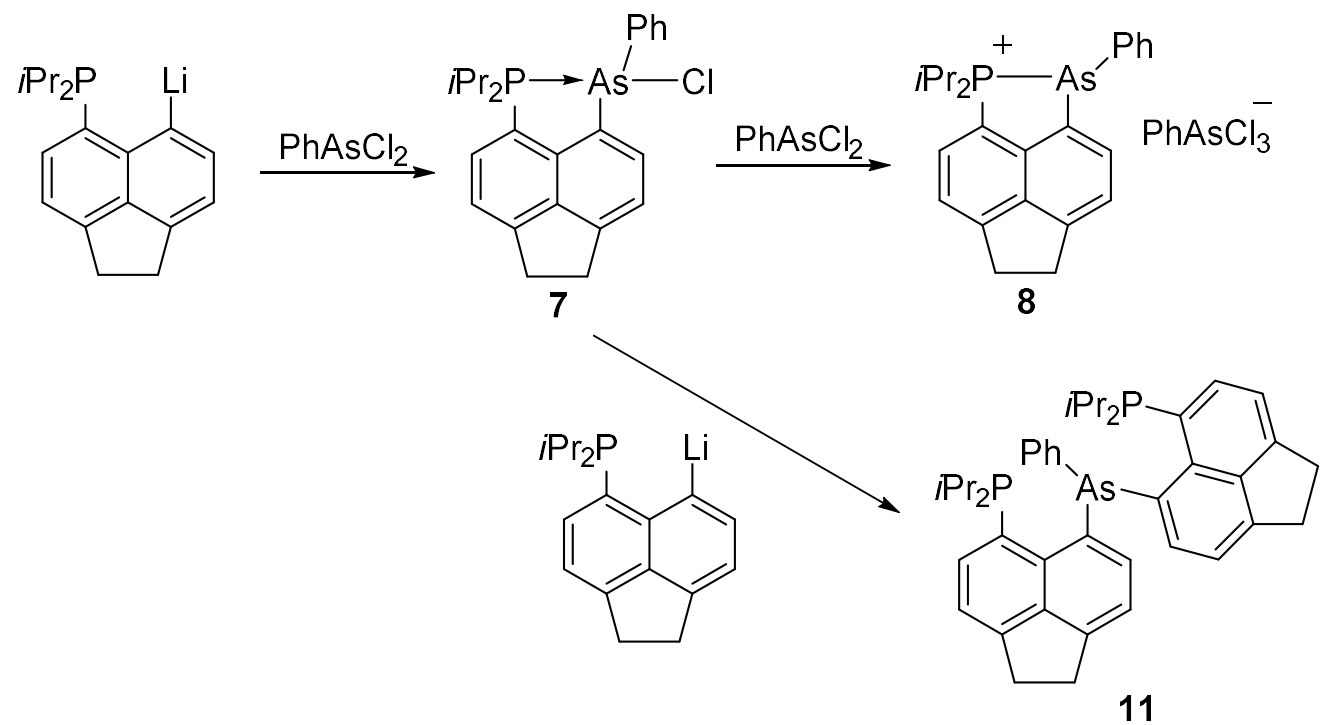

Scheme S2: Formation of a mixture of 7, 8, and soluble dimer 11, from the reaction of $A c e n a p\left(\operatorname{PiPr}_{2}\right)\left(\mathrm{Li}_{)}\right)$with $\mathrm{PhAsCl} 2$ 

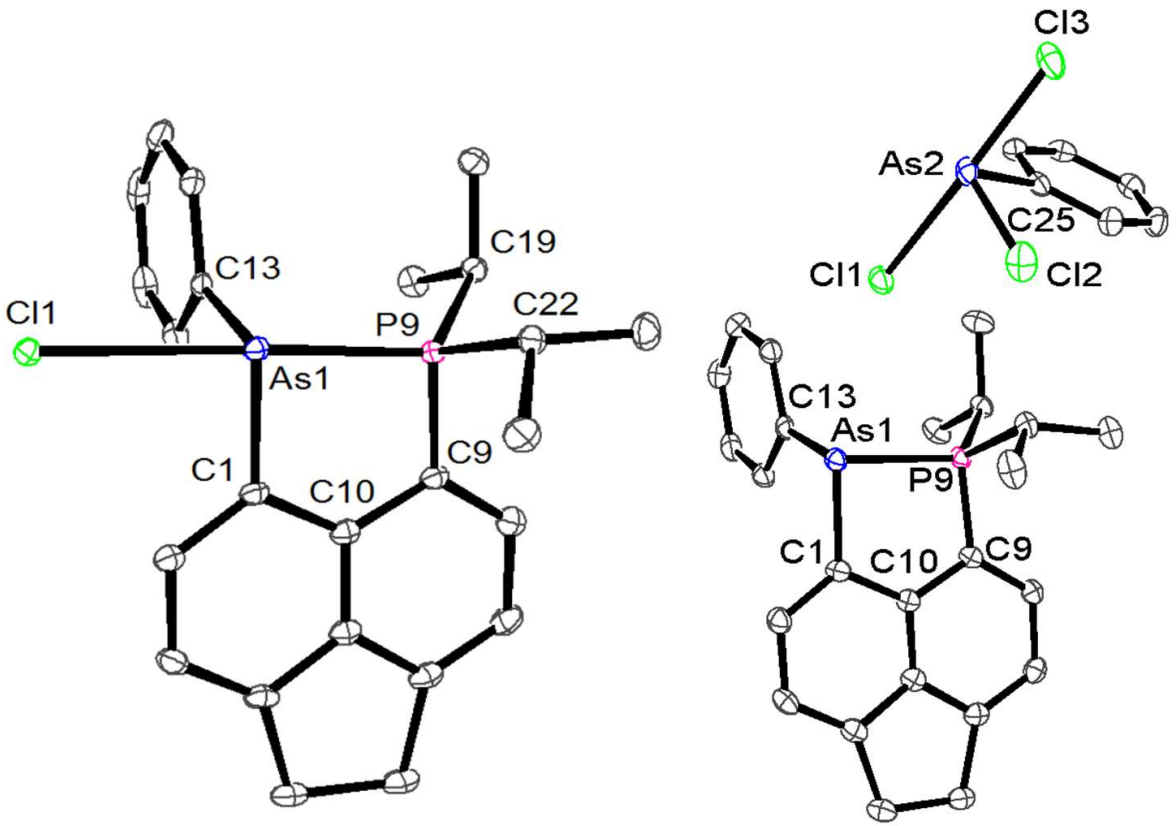

Figure S1: Structures of $\mathbf{7}$ (left) and $\mathbf{8}$ (right) in the solid state. Carbon-bound hydrogen atoms omitted for clarity.

Table S2: Selected distances $(\AA \AA)$ and angles $\left({ }^{\circ}\right)$ for $\mathbf{7}$ and $\mathbf{8}$.

\begin{tabular}{|c|c|c|c|}
\hline \multicolumn{4}{|l|}{4.7} \\
\hline C1-As1 & $1.978(2)$ & C9-P9 & $1.798(2)$ \\
\hline As1-P9 & $2.4239(7)$ & As1-Cl1 & $2.9016(8)$ \\
\hline out-of-plane displacement (As1) & 0.154 & out-of-plane displacement (P9) & 0.224 \\
\hline P9-As1-Cl1 & $175.10(2)$ & P9-As1-C13 & $93.33(5)$ \\
\hline As1-C1‥C9-P9 & $2.37(7)$ & Splay angle & $-4.3(3)$ \\
\hline \multicolumn{4}{|l|}{4.8} \\
\hline C1-As1 & $1.963(2)$ & C9-P9 & $1.806(2)$ \\
\hline As1-P9 & $2.3598(6)$ & As2-Cl1 & $2.5969(8)$ \\
\hline As2-Cl2 & $2.2065(6)$ & As2-Cl3 & $2.4031(8)$ \\
\hline out-of-plane displacement (As1) & 0.148 & out-of-plane displacement (P9) & 0.163 \\
\hline $\mathrm{Cl} 1-\mathrm{As} 2-\mathrm{Cl} 2$ & $91.25(2)$ & $\mathrm{Cl} 2-\mathrm{As} 2-\mathrm{Cl} 3$ & $93.26(2)$ \\
\hline $\mathrm{Cl} 1-\mathrm{As} 2-\mathrm{Cl} 3$ & $174.62(3)$ & Splay angle & $-5.6(3)$ \\
\hline
\end{tabular}

Compound 7 shows an interesting solid-state structure (Figure S1, Table S2), with a $3 c-4 \mathrm{e}^{-}$interaction between the $\mathrm{P}, \mathrm{As}$ and $\mathrm{Cl}$ atoms. This is indicated by the near-linear $\mathrm{P}-\mathrm{As}-\mathrm{Cl}$ arrangement (bond angle = $\left.175.10(2)^{\circ}\right)$ and $\mathrm{As}-\mathrm{Cl}$ distance of $2.9016(8) \AA$. This is substantially longer than a typical As-Cl bond, ${ }^{8}$ but within the sum of the van der Waals radii of the two atoms $(3.60 \AA) .{ }^{9}$ This compound straddles the boundary between a molecular donor-acceptor complex and an ionic phosphonium salt. While this $3 \mathrm{c}-4 \mathrm{e}^{-}$ bond exists in the solid state, it is likely that compound $\mathbf{7}$ dissociates into ions in polar solvents (Scheme S3). 


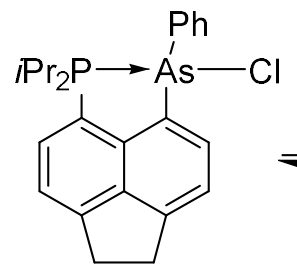

7

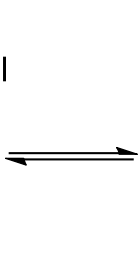

(l)

Scheme S3: Possible dissociation equilibrium for compound $\mathbf{7}$ in solution.

Unlike 7, compound $\mathbf{8}$ is a fully ionic phosphonium salt in the solid state (Figure S1, Table S2). This is due to the $\mathrm{PhAsCl}_{3}{ }^{-}$counterion, which is less strongly coordinating than the $\mathrm{Cl}^{-}$ion. The $\mathrm{PhAsCl}_{3}{ }^{-}$ion adopts a "see-saw" geometry, with the axial As-Cl bonds being significantly elongated (As2-Cl1 2.5969(8) $\AA$; As2$\mathrm{Cl} 32.4031(8) \AA$ ) compared to the equatorial As-Cl bond (As2-Cl2 2.2065(6) $\AA$ ). There is a slight decrease in the P9-As1 distance on going from 7 to 8 (7: 2.4239(7) $\AA$; 8: 2.3598(6) $\AA$ ), corresponding to the switch from a $3 \mathrm{c}-4 \mathrm{e}^{-}$interaction in $\mathbf{7}$ to a formal covalent bond in $\mathbf{8}$.

\section{S4 - Synthesis}

\section{$\mathrm{S} 4.1-\mathrm{Nap}(\mathrm{PH})_{2}(2)$}
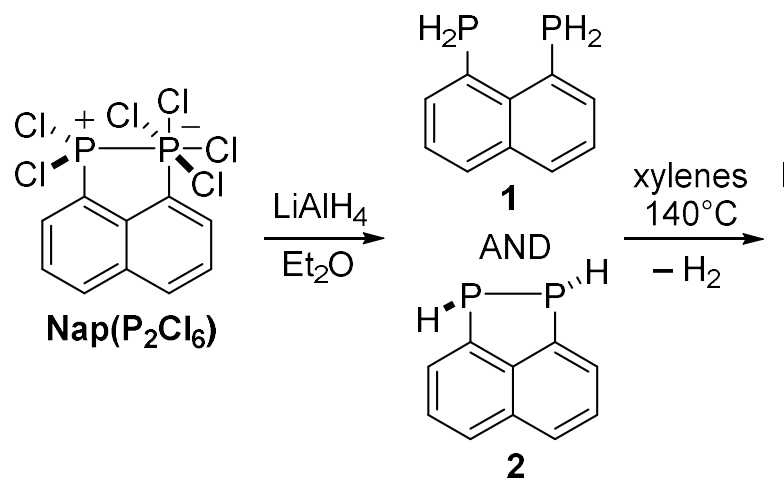<smiles>[PH]Pc1cccc2ccccc12</smiles>

Scheme S4: Synthesis of compound 2.

To a stirred suspension of $\mathrm{LiAlH}_{4}(1.13 \mathrm{~g}, 29.8 \mathrm{mmol})$ in diethyl ether $(100 \mathrm{~mL})$, cooled to $-78{ }^{\circ} \mathrm{C}$, a stirred suspension of $\mathrm{Nap}\left(\mathrm{P}_{2} \mathrm{Cl}_{6}\right)(1.00 \mathrm{~g}, 2.49 \mathrm{mmol})$ in diethyl ether $(50 \mathrm{~mL})$ was added dropwise via cannula. The reaction was allowed to warm to room temperature, with stirring, overnight. The reaction mixture was cooled to $0{ }^{\circ} \mathrm{C}$ and degassed water $(5 \mathrm{~mL})$ was added cautiously. Insoluble salts were removed by filtration and washed with diethyl ether $(50 \mathrm{~mL})$. Volatiles were removed in vacuo to afford $\mathrm{Nap}(\mathrm{PH})_{2}$ as an off white solid, containing approximately $26 \% \mathrm{Nap}\left(\mathrm{PH}_{2}\right)_{2}$ as an impurity $(0.282 \mathrm{~g})$.

A sample of this mixture $(140 \mathrm{mg})$ was dissolved in xylenes $(15 \mathrm{~mL})$ and heated under reflux for 5 days. After this time, volatiles were removed in vacuo to afford pure $\mathrm{Nap}(\mathrm{PH})_{2}$ as an off-white oil, which crystallised to a low melting solid on standing at room temperature $(139 \mathrm{mg}, 0.731 \mathrm{mmol}, 59 \%$ overall yield from $\mathrm{Nap}\left(\mathrm{P}_{2} \mathrm{Cl}_{6}\right)$ ). Crystals suitable for single crystal X-ray diffraction were obtained by melting and recrystallisation of the solid between $5-40{ }^{\circ} \mathrm{C}$.

The full NMR assignment of this compound is discussed in section S5. 
$\mathrm{mp} 38-39^{\circ} \mathrm{C}$.

IR (Nujol mull) $\bar{v}_{\text {max }} 3054$ (m, CH, aromatic), 2264 (s, PH), 1731 (m), 1485 (m), 1264 (m), 917 (m), 799 (s, PH wag), $762(\mathrm{~m}), 723(\mathrm{w}) \mathrm{cm}^{-1}$

MS (APCl+): m/z (\%) $189.00(58)\left[\mathrm{M}^{+}-\mathrm{H}\right], 190.01(43)\left[\mathrm{M}^{+}\right], 191.02(89)\left[\mathrm{M}+\mathrm{H}^{+}\right], 207.01(100)[\mathrm{M}+\mathrm{O}+$ $\left.\mathrm{H}^{+}\right]$.

HRMS (APCl+): $m / z\left[M+\mathrm{H}^{+}\right]$calcd for $\mathrm{C}_{10} \mathrm{H}_{9} \mathrm{P}_{2}, 191.0179$; found, 191.0175 .

\section{S4.2 - Acenap(PiPr2)(P(H)Ph) (5b)}

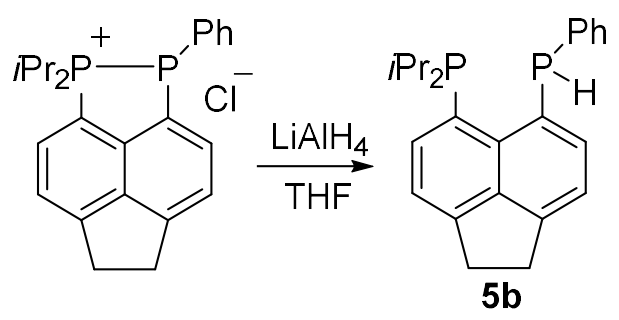

Scheme S5: Synthesis of compound $\mathbf{5 b}$

Synthesis adapted from method published by Kilian et al. ${ }^{3,5}$

To a stirred suspension of [Acenap(PiPr $)(\mathrm{PPh})][\mathrm{Cl}](4.08 \mathrm{~g}, 9.88 \mathrm{mmol})$ in THF $(100 \mathrm{~mL})$, cooled to $0{ }^{\circ} \mathrm{C}$, a suspension of $\mathrm{LiAlH}_{4}(0.450 \mathrm{~g}, 11.9 \mathrm{mmol})$ in THF $(20 \mathrm{~mL})$ was added in portions over 30 minutes. The reaction was allowed to warm to room temperature and stirred for 1 hour. The reaction was cooled to 0 ${ }^{\circ} \mathrm{C}$ and degassed water $(2 \mathrm{~mL})$ was added cautiously.

The reaction was filtered to remove insoluble impurities and the solid was washed with THF (10 mL). Volatiles were removed in vacuo and the resulting solid dried in vacuo for 2 hours. Hexane $(100 \mathrm{~mL})$ was added and the resulting suspension filtered to remove salts. Volatiles were removed in vacuo to yield crude $5 \mathbf{b}$ as a yellow solid $(3.11 \mathrm{~g}, 8.22 \mathrm{mmol}, 83 \%)$, which was contaminated with a small quantity $(<5 \%)$ of the $\mathrm{P}-\mathrm{P}$ bonded compound $\mathbf{6 b}$.

Recrystallisation from hot acetonitrile afforded analytically pure $\mathbf{5 b}$ as a yellow crystalline solid $(2.03 \mathrm{~g}$, $5.36 \mathrm{mmol}, 54 \%)$, which was used in subsequent kinetic studies.

Crystals suitable for single crystal X-ray diffraction were grown from slow diffusion of methanol into a concentrated solution of $\mathbf{5 b}$ in DCM at room temperature. The ${ }^{1} \mathrm{H}$ and ${ }^{31} \mathrm{P}\left\{{ }^{1} \mathrm{H}\right\} \mathrm{NMR}$ of the product are in good agreement with previously published data. ${ }^{3}$

mp $100-103^{\circ} \mathrm{C}$.

${ }^{1} \mathrm{H}$ NMR $\left(270 \mathrm{MHz}, \mathrm{CDCl}_{3}, \delta\right): 7.79-7.73(\mathrm{~m}, 1 \mathrm{H}, 2-\mathrm{H}), 7.65\left(\mathrm{dd},{ }^{3} \mathrm{~J}_{\mathrm{HH}}=7.2 \mathrm{~Hz},{ }^{3} J_{\mathrm{HP}}=3.1 \mathrm{~Hz}, 1 \mathrm{H}, 8-\mathrm{H}\right), 7.43-$ $7.31(\mathrm{~m}, 3 \mathrm{H}, 3-\mathrm{H}, o-\mathrm{Ph} \mathrm{CH}), 7.28-7.18(\mathrm{~m}, 4 \mathrm{H}, 7-\mathrm{H}, \mathrm{m} / \mathrm{p}-\mathrm{Ph} \mathrm{CH}), 5.71\left(\mathrm{dd},{ }^{1} J_{\mathrm{HP}}=202.8 \mathrm{~Hz},{ }^{5} \mathrm{JP}_{\mathrm{HP}}=57.9 \mathrm{~Hz}, 1 \mathrm{H}\right.$, $\mathrm{P}(\mathrm{Ph}) \mathrm{H}), 3.40$ (br s, $4 \mathrm{H}, 11-\mathrm{H}, 12-\mathrm{H}), 2.22-2.02(\mathrm{~m}, 2 \mathrm{H}, 2 \times i \operatorname{Pr} \mathrm{CH}), 1.14\left(\mathrm{dd},{ }^{3} J_{\mathrm{HP}}=13.4 \mathrm{~Hz},{ }^{3} \mathrm{~J}_{\mathrm{HH}}=7.0 \mathrm{~Hz}\right.$, $\left.3 \mathrm{H}, i \operatorname{PrCH})_{3}\right), 1.06-0.95\left(\mathrm{~m}, 6 \mathrm{H}, 2 \times i \mathrm{PrCH}_{3}\right), 0.42\left(\mathrm{dd},{ }^{3} \mathrm{JP}_{\mathrm{HP}}=13.3 \mathrm{~Hz},{ }^{3} \mathrm{JHH}_{\mathrm{HH}}=7.0 \mathrm{~Hz}, 3 \mathrm{H}, i \mathrm{PrCH}_{3}\right)$.

${ }^{31} \mathrm{P}\left\{{ }^{1} \mathrm{H}\right\}$ NMR $\left(109 \mathrm{MHz}, \mathrm{CDCl}_{3}, \delta\right):-11.7\left(\mathrm{~d}, \mathrm{PiPr}_{2}\right),-40.4(\mathrm{~d}, \mathrm{P}(\mathrm{Ph}) \mathrm{H}),{ }^{4} \mathrm{JPP}_{\mathrm{PP}}=169 \mathrm{~Hz}$. 


\section{S4.3 - Acenap(PiPr2)(P(D)Ph) (5d)}

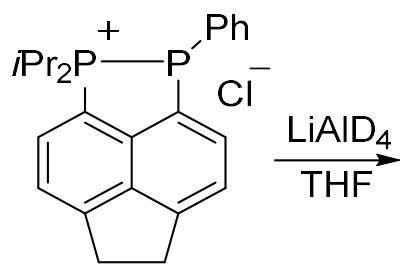<smiles>[2H]P(c1ccccc1)c1ccc2c3c(ccc(PC(C)C)c13)C2</smiles>

Scheme S6: Synthesis of compound 5d

To a flask charged with a magnetic stirrer, [Acenap(PiPr $)(\mathrm{PPh})][\mathrm{Cl}](700 \mathrm{mg}, 1.70 \mathrm{mmol})$, and $\mathrm{LiAlD}_{4}(78$ $\mathrm{mg}, 1.86 \mathrm{mmol})$; cold $\left(0^{\circ} \mathrm{C}\right) \mathrm{THF}(20 \mathrm{~mL})$ was added cautiously. The reaction was stirred at room temperature for $30 \mathrm{mins}$, then cooled to $0{ }^{\circ} \mathrm{C}$. Degassed $\mathrm{D}_{2} \mathrm{O}(1 \mathrm{~mL})$ was added dropwise to quench the reaction.

Volatiles were removed in vacuo, and hexane $(40 \mathrm{~mL})$ was added to the resulting solid. The reaction mixture was filtered to remove salts, and volatiles removed in vacuo to afford compound $\mathbf{5} \mathbf{d}$ as a yellow solid (445 mg, $1.17 \mathrm{mmol}, 69 \%$ ). The compound was contaminated with some of the non-deuterated $\mathbf{5 b}$ (approximately 10\%).

${ }^{1} \mathrm{H}$ NMR $\left(400 \mathrm{MHz}, \mathrm{CDCl}_{3}, \delta\right): 7.75\left(\mathrm{dd},{ }^{3} \mathrm{~J}_{\mathrm{HH}}=7.1 \mathrm{~Hz},{ }^{3} \mathrm{~J}_{\mathrm{HP}}=5.5,1 \mathrm{H}, 2-\mathrm{H}\right), 7.64\left(\mathrm{dd},{ }^{3} J_{\mathrm{HH}}=7.2 \mathrm{~Hz},{ }^{3} \mathrm{JPP}_{\mathrm{HP}}=3.2\right.$, $1 \mathrm{H}, 8-\mathrm{H}) 7.41-7.35(\mathrm{~m}, 2 \mathrm{H}, o-\mathrm{Ph} \mathrm{CH}), 7.33\left(\mathrm{~d},{ }^{3} \mathrm{~J}_{\mathrm{HH}}=7.2 \mathrm{~Hz}, 1 \mathrm{H}, \mathrm{H}-3\right), 7.26-7.18(\mathrm{~m}, 4 \mathrm{H}, 7-\mathrm{H}, \mathrm{m} / \mathrm{p}-\mathrm{Ph} \mathrm{CH})$, $3.39(\mathrm{~s}, 4 \mathrm{H}, 11-\mathrm{H}, 12-\mathrm{H}), 2.20-2.05(\mathrm{~m}, 2 \mathrm{H}, 2 \times i \operatorname{Pr} \mathrm{CH}), 1.13\left(\mathrm{dd},{ }^{3} \mathrm{JP}_{\mathrm{HP}}=13.4 \mathrm{~Hz},{ }^{3} \mathrm{~J}_{\mathrm{HH}}=7.0 \mathrm{~Hz}, 3 \mathrm{H}, i \operatorname{Pr} \mathrm{CH}_{3}\right)$, $1.05-0.94\left(\mathrm{~m}, 6 \mathrm{H}, 2 \times i \operatorname{Pr} \mathrm{CH}_{3}\right), 0.41\left(\mathrm{dd},{ }^{3} J_{\mathrm{HP}}=13.3 \mathrm{~Hz},{ }^{3} J_{\mathrm{HH}}=7.0 \mathrm{~Hz}, 3 \mathrm{H}, i \mathrm{Pr} \mathrm{CH}_{3}\right)$.

${ }^{31} \mathrm{P}\left\{{ }^{1} \mathrm{H}\right\}$ NMR $\left(162 \mathrm{MHz}, \mathrm{CDCl}_{3}, \delta\right):-12.4\left(\mathrm{dt},{ }^{4} J_{\mathrm{PP}}=168 \mathrm{~Hz},{ }^{5} \mathrm{JP}_{\mathrm{PD}}=8.4 \mathrm{~Hz}, \mathrm{PiPr} 2\right),-42.4\left(\mathrm{dt},{ }^{4} J_{\mathrm{PP}}=168 \mathrm{~Hz},{ }^{1} J_{\mathrm{PD}}\right.$ $=31 \mathrm{~Hz})$.

${ }^{2} \mathrm{H}\left\{{ }^{1} \mathrm{H}\right\}$ NMR $(77 \mathrm{MHz}$, xylenes, $\delta): 5.84\left(\mathrm{dd},{ }^{1} J_{D P}=31 \mathrm{~Hz},{ }^{5} J_{D P}=9.1 \mathrm{~Hz}\right)$.

\section{S4.4 - Acenap(PiPr)(PH) (6a)}<smiles>CC(C)c1ccc2c3c(ccc(P)c13)CC2</smiles>

$5 a$

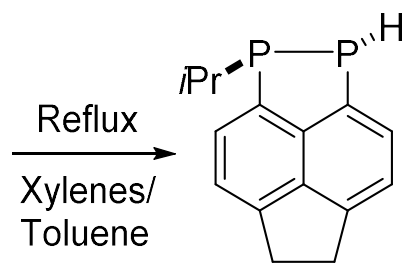

$6 a$

Scheme S7: Synthesis of compound 6a

Compound 5a ( $0.73 \mathrm{~g}, 2.4 \mathrm{mmol})$ was dissolved in toluene $(20 \mathrm{~mL})$ and heated under reflux for 3 days. Alternatively, it was dissolved in xylenes $(20 \mathrm{~mL})$ and heated under reflux for $8 \mathrm{~h}$. Volatiles were removed in vacuo to yield $6 a$ as a viscous red oil $(0.62 \mathrm{~g}, 2.4 \mathrm{mmol},>99 \%)$. An analytically pure white solid was obtained from $\mathrm{MeCN}$ at $2{ }^{\circ} \mathrm{C}$. 
Anal Calcd for $\mathrm{C}_{15} \mathrm{H}_{16} \mathrm{P}_{2}: \mathrm{C}, 69.77 ; \mathrm{H}, 6.25$. Found: $\mathrm{C}, 69.85 ; \mathrm{H}, 6.28$.

IR (KBr disc) $\bar{\nu}_{\text {max: }} 2962(\mathrm{~m}), 2916(\mathrm{~m}, \mathrm{CH}), 2362(\mathrm{~m}, \mathrm{PH}), 1606(\mathrm{~m}), 1566(\mathrm{~m}), 1449(\mathrm{~m}), 1384(\mathrm{~m}), 1261$ (m), $1213(\mathrm{~m}), 841(\mathrm{~m}) \mathrm{cm}^{-1}$.

Raman (sealed capillary) $\bar{v}_{\max }: 3340$ (m, CH, aromatic), 2920 (m, CH, aliphatic), $1608(\mathrm{~s}), 1500$ (m), 1415 (s), $1412(\mathrm{~m}), 1035(\mathrm{~m}), 835(\mathrm{~m}), 721(\mathrm{~m}), 595(\mathrm{~m}) \mathrm{cm}^{-1}$

${ }^{1} \mathrm{H}$ NMR $\left(400 \mathrm{MHz}, \mathrm{C}_{6} \mathrm{D}_{6}, \delta\right): 7.61\left(\mathrm{dd},{ }^{3} J_{\mathrm{HH}}=6.9 \mathrm{~Hz},{ }^{3} J_{\mathrm{HP}}=4.9 \mathrm{~Hz}, 1 \mathrm{H}, 8-\mathrm{H}\right), 7.57\left(\mathrm{ddd},{ }^{3} J_{\mathrm{HH}}=7.3 \mathrm{~Hz},{ }^{3} J_{\mathrm{HP}}=\right.$ $\left.5.9 \mathrm{~Hz},{ }^{4} \mathrm{~J}_{\mathrm{HH}}=1.9 \mathrm{~Hz}, 1 \mathrm{H}, 2-\mathrm{H}\right), 7.05\left(\mathrm{ddt},{ }^{3} J_{\mathrm{HH}}=7.1 \mathrm{~Hz},{ }^{4} \mathrm{~J}_{\mathrm{HP}}=2.2 \mathrm{~Hz},{ }^{4} J_{\mathrm{HH}}=1.2 \mathrm{~Hz}, 1 \mathrm{H}, 7-\mathrm{H}\right), 7.00\left(\mathrm{dt},{ }^{3} \mathrm{~J}_{\mathrm{HH}}=\right.$ $\left.7.1 \mathrm{~Hz},{ }^{4} J_{\mathrm{HH}}=1.6 \mathrm{~Hz}, 1 \mathrm{H}, 3-\mathrm{H}\right), 4.40\left(\mathrm{ddd},{ }^{1} J_{\mathrm{HP}}=204 \mathrm{~Hz},{ }^{2} J_{\mathrm{HP}}=18.4 \mathrm{~Hz},{ }^{4} J_{\mathrm{HH}}=1.9 \mathrm{~Hz}, 1 \mathrm{H}, \mathrm{PH}\right), 2.93(\mathrm{~s}, 4 \mathrm{H}, \mathrm{H}-$ $11, \mathrm{H}-12), 1.77-1.63(\mathrm{~m}, 1 \mathrm{H}, i \mathrm{Pr} \mathrm{CH}), 1.05\left(\mathrm{dd},{ }^{3} \mathrm{~J}_{\mathrm{HP}}=13.9 \mathrm{~Hz},{ }^{3} J_{\mathrm{HH}}=7.0 \mathrm{~Hz}, 3 \mathrm{H}, i \operatorname{Pr} \mathrm{CH}_{3}\right), 1.02\left(\mathrm{dd},{ }^{3} \mathrm{JP}_{\mathrm{HP}}=14.2\right.$ $\left.\mathrm{Hz},{ }^{3} \mathrm{JHH}_{\mathrm{HH}}=6.9 \mathrm{~Hz}, 3 \mathrm{H}, i \mathrm{Pr} \mathrm{CH}_{3}\right)$.

${ }^{13} \mathrm{C}\left\{{ }^{1} \mathrm{H}\right\}$ NMR (75 MHz, $\left.\mathrm{C}_{6} \mathrm{D}_{6}, \delta\right): 146.1$ (s, qC-4/qC-6), 145.8 (s, qC-4/qC-6), 143.4 (s, qC-10), 139.7 (dd, ${ }^{1} \mathrm{JP}_{\mathrm{CP}}$ $\left.=30.7 \mathrm{~Hz},{ }^{2} J_{\mathrm{CP}}=2.7 \mathrm{~Hz}, \mathrm{qC}-9\right), 139.6$ (br s, qC-5), 132.8 (dd, ${ }^{1} \mathrm{~J}_{\mathrm{CP}}=24.1 \mathrm{~Hz},{ }^{2} J_{\mathrm{CP}}=1.2 \mathrm{~Hz}, \mathrm{qC}-1$ ), 131.9 (dd, $\left.{ }^{2} J_{\mathrm{CP}}=21.4 \mathrm{~Hz},{ }^{3} J_{\mathrm{CP}}=1.6 \mathrm{~Hz}, \mathrm{C}-2 / \mathrm{C}-8\right), 131.7\left(\mathrm{~d},{ }^{2} \mathrm{~J}_{\mathrm{CP}}=22.4 \mathrm{~Hz}, \mathrm{C}-2 / \mathrm{C}-8\right), 120.7\left(\mathrm{~d},{ }^{3} \mathrm{JPP}_{\mathrm{CP}}=7.2 \mathrm{~Hz}, \mathrm{C}-3\right.$ ), 120.3 $\left(\mathrm{d},{ }^{3} \mathrm{~J}_{\mathrm{CP}}=6.8 \mathrm{~Hz}, \mathrm{C}-7\right), 30.8(\mathrm{~s}, \mathrm{C}-11 / \mathrm{C}-12), 30.7(\mathrm{~s}, \mathrm{C}-11 / \mathrm{C}-12), 28.9$ (dd, $\left.{ }^{1} \mathrm{~J}_{\mathrm{CP}}=16.5 \mathrm{~Hz},{ }^{2} \mathrm{~J}_{\mathrm{CP}}=14.6 \mathrm{~Hz}, i \operatorname{Pr~CH}\right)$, $20.2\left(\mathrm{~m}, 2 \times i \mathrm{Pr} \mathrm{CH}_{3}\right)$.

${ }^{31}$ P NMR $\left(162 \mathrm{MHz}, \mathrm{C}_{6} \mathrm{D}_{6}, \delta\right):-7.4\left(\mathrm{dm},{ }^{1} J_{\mathrm{PP}}=203 \mathrm{~Hz}, \mathrm{iPrP}\right),-89.7\left(\approx \mathrm{tt},{ }^{1} \mathrm{JPP}_{\mathrm{PP}}=203 \mathrm{~Hz},{ }^{1} \mathrm{JPH}_{\mathrm{PH}}=204 \mathrm{~Hz},{ }^{3} \mathrm{JPH}_{\mathrm{PH}}=6.6\right.$ $\mathrm{Hz}, \mathrm{PH})$.

${ }^{31} \mathrm{P}\left\{{ }^{1} \mathrm{H}\right\} \mathrm{NMR} \delta_{\mathrm{P}}\left(162 \mathrm{MHz}, \mathrm{C}_{6} \mathrm{D}_{6}, \delta\right):-7.4\left(\mathrm{~d},{ }^{1} J_{\mathrm{PP}}=203 \mathrm{~Hz}, i \mathrm{PrP}\right),-89.7\left(\mathrm{~d},{ }^{1} J_{\mathrm{PP}}=203 \mathrm{~Hz}, \mathrm{PH}\right)$.

MS (ES+): $m / z(\%) 275.07(72)\left[\mathrm{M}+\mathrm{O}+\mathrm{H}^{+}\right], 297.06(100)\left[\mathrm{M}+\mathrm{O}+\mathrm{Na}^{+}\right]$.

HRMS (ES+): $m / z\left[\mathrm{M}+\mathrm{O}+\mathrm{Na}^{+}\right]$calcd for $\mathrm{C}_{15} \mathrm{H}_{16} \mathrm{ONaP}_{2}, 297.0574$; found, 297.0566.

S4.5 - Acenap(PiPr)(PPh) (6b)<smiles>CC(C)Pc1ccc2c3c(ccc(P)c13)CC2</smiles>

$5 b$

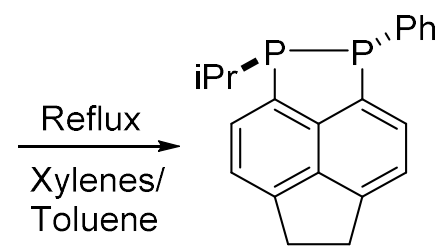

$6 b$

Scheme S8: Synthesis of compound $\mathbf{6 b}$.

Compound $\mathbf{5 b}(0.59 \mathrm{~g}, 1.6 \mathrm{mmol})$ was dissolved in toluene $(10 \mathrm{~mL})$ and heated under reflux for 3 days. Alternatively, it was dissolved in xylenes $(10 \mathrm{~mL})$ and heated under reflux for $8 \mathrm{~h}$. Volatiles were removed in vacuo to yield $6 \mathrm{~b}$ as a yellow solid $(0.59 \mathrm{~g}, 1.6 \mathrm{mmol},>99 \%)$. 
Crystals suitable for single crystal X-ray diffraction were grown from slow diffusion of ethanol into a concentrated solution of $\mathbf{6 b}$ in DCM at room temperature.

$\mathrm{mp} 98-101^{\circ} \mathrm{C}$.

Anal Calcd for $\mathrm{C}_{21} \mathrm{H}_{20} \mathrm{P}_{2}: \mathrm{C}, 75.44 ; \mathrm{H}, 6.03$. Found: $\mathrm{C}, 75.35 ; \mathrm{H}, 5.95$.

IR ( $\mathrm{KBr}$ disk) $\bar{\nu}_{\text {max }}: 3068(\mathrm{~m}, \mathrm{CH}$, aromatic), $2943(\mathrm{~s}), 2922$ (s, CH, aliphatic), $2857(\mathrm{~s}), 1607(\mathrm{~m}), 1582(\mathrm{~s})$, $1569(\mathrm{~m}), 1481(\mathrm{~s}), 1450$ (s), 1433 (s), 1384 (vs), 1215 (s), 1176 (s), 1152 (s), 1026 (s), 842 (vs), 816 (s), 740 (vs) $\mathrm{cm}^{-1}$

${ }^{1} \mathrm{H}$ NMR $\left(500 \mathrm{MHz}, \mathrm{CDCl}_{3}, \delta\right): 7.79\left(\mathrm{dd},{ }^{3} \mathrm{~J}_{\mathrm{HH}}=6.9 \mathrm{~Hz},{ }^{3} \int_{\mathrm{HP}}=5.6 \mathrm{~Hz}, 1 \mathrm{H}, 2-\mathrm{H}\right), 7.67\left(\mathrm{dd},{ }^{3} \int_{\mathrm{HH}}=6.9 \mathrm{~Hz},{ }^{3} J_{\mathrm{HP}}=4.9\right.$ $\mathrm{Hz}, 1 \mathrm{H}, 8-\mathrm{H}), 7.40\left(\mathrm{dd},{ }^{3} J_{\mathrm{HH}}=6.9 \mathrm{~Hz},{ }^{4} J_{\mathrm{HP}}=1.8 \mathrm{~Hz}, 1 \mathrm{H}, 3-\mathrm{H}\right), 7.36\left(\mathrm{dd},{ }^{3} J_{\mathrm{HH}}=6.9 \mathrm{~Hz},{ }^{4} J_{\mathrm{HP}}=1.4 \mathrm{~Hz}, 1 \mathrm{H}, 7-\mathrm{H}\right)$, 7.23-7.18 (m, 2H, o-Ph CH), 7.18-7.15 (m, 2H, m/p-Ph CH), $3.48(\mathrm{~s}, 4 \mathrm{H}, 11-\mathrm{H}, 12-\mathrm{H}), 1.93$ (dsept, ${ }^{2} J_{\mathrm{HP}}=7.0$ $\left.\mathrm{Hz},{ }^{3} J_{\mathrm{HH}}=7.0 \mathrm{~Hz}, 1 \mathrm{H}, i \operatorname{PrCH}\right), 1.19\left(\mathrm{dd},{ }^{3} J_{\mathrm{HP}}=14.7 \mathrm{~Hz},{ }^{3} J_{\mathrm{HH}}=7.0 \mathrm{~Hz}, 3 \mathrm{H}, i \operatorname{Pr} \mathrm{CH}_{3}\right), 1.10\left(\mathrm{dd},{ }^{3} J_{\mathrm{HP}}=14.1 \mathrm{~Hz},{ }^{3} J_{\mathrm{HH}}\right.$ $=6.9 \mathrm{~Hz}, 3 \mathrm{H}, i \mathrm{Pr} \mathrm{CH}$ ).

${ }^{13} \mathrm{C}\left\{{ }^{1} \mathrm{H}\right\}$ NMR (126 MHz, CDCl $\left.3, \delta\right): 146.4$ (s, qC-4), 146.0 (s, qC-6), 141.6 (d, $\left.{ }^{2} J_{\mathrm{CP}}=1.6 \mathrm{~Hz}, \mathrm{qC}-10\right), 139.2$ (s, qC-5), 138.7 (dd, $\left.{ }^{1} J_{\mathrm{CP}}=19.1 \mathrm{~Hz},{ }^{2} J_{\mathrm{CP}}=11.2 \mathrm{~Hz}, i-\mathrm{Ph} \mathrm{qC}\right), 136.8$ (dd, ${ }^{1} J_{\mathrm{CP}}=31.7 \mathrm{~Hz},{ }^{2} J_{\mathrm{CP}}=2.5 \mathrm{~Hz}, \mathrm{qC}-9$ ), 136.1 $\left(\mathrm{dd},{ }^{1} \mathrm{~J}_{\mathrm{CP}}=23.3 \mathrm{~Hz},{ }^{2} \mathrm{~J}_{\mathrm{CP}}=1.9 \mathrm{~Hz}, \mathrm{qC}-1\right), 132.5\left(\mathrm{~d},{ }^{2} \mathrm{~J}_{\mathrm{CP}}=21.6 \mathrm{~Hz}, \mathrm{C}-2\right), 131.9(\mathrm{~m}, \mathrm{C}-8, o-\mathrm{Ph} \mathrm{CH}), 128.3\left(\mathrm{~d},{ }^{3} \mathrm{~J}_{\mathrm{CP}}\right.$ $=6.6 \mathrm{~Hz}, m-\mathrm{Ph} \mathrm{CH}), 127.9\left(\mathrm{~d},{ }^{4} \mathrm{~J}_{\mathrm{CP}}=1.8 \mathrm{~Hz}, \mathrm{C}-16\right), 120.6\left(\mathrm{~d},{ }^{3} \mathrm{~J}_{\mathrm{CP}}=6.9 \mathrm{~Hz}, \mathrm{C}-3\right), 120.5\left(\mathrm{~d},{ }^{3} \mathrm{~J}_{\mathrm{CP}}=6.4 \mathrm{~Hz}, \mathrm{C}-7\right)$, $30.8(\mathrm{~s}, \mathrm{C}-11), 30.7(\mathrm{~s}, \mathrm{C}-12), 28.6\left(\approx \mathrm{t},{ }^{1} J_{\mathrm{CP}}=15.5 \mathrm{~Hz},{ }^{2} \mathrm{~J}_{\mathrm{CP}}=15.5 \mathrm{~Hz}, \mathrm{PPr} \mathrm{CH}\right), 20.7\left(\mathrm{dd},{ }^{2} J_{\mathrm{CP}}=13.5 \mathrm{~Hz},{ }^{3} J_{\mathrm{CP}}=\right.$ $\left.10.1 \mathrm{~Hz}, i \mathrm{PrCH} \mathrm{CH}_{3}\right), 20.2\left(\mathrm{dd},{ }^{2} \mathrm{~J}_{\mathrm{CP}}=12.9 \mathrm{~Hz},{ }^{3} J_{\mathrm{CP}}=8.1 \mathrm{~Hz}, i \mathrm{PrCH} \mathrm{CH}_{3}\right)$

${ }^{31} \mathrm{P}$ NMR $(203 \mathrm{MHz}, \mathrm{CDCl} 3, \delta): 20.0$ (dseptd, $\left.{ }^{1} \mathrm{JP}_{\mathrm{PP}}=240 \mathrm{~Hz},{ }^{3} \mathrm{PHH}_{\mathrm{PH}}=14.4 \mathrm{~Hz},{ }^{2} J_{\mathrm{PH}}=7.0 \mathrm{~Hz}, i \mathrm{PrP}\right),-14.7$ (dt, ${ }^{1} J_{\mathrm{PP}}$ $\left.=240 \mathrm{~Hz},{ }^{3} \mathrm{JHH}_{\mathrm{PH}}=6.5 \mathrm{~Hz}, \mathrm{PPh}\right)$.

${ }^{31} \mathrm{P}\left\{{ }^{1} \mathrm{H}\right\}$ NMR $(203 \mathrm{MHz}, \mathrm{CDCl} 3, \delta): 20.0(\mathrm{~d}, \mathrm{PPrP}),-14.7(\mathrm{~d}, \mathrm{PPh}),{ }^{1}{ }_{\mathrm{PP}}=240 \mathrm{~Hz}$.

MS (EI): $m / z(\%) 257.05(18)\left[\mathrm{M}^{+}-\mathrm{Ph}\right], 291.04(100)\left[\mathrm{M}^{+}-i \mathrm{Pr}\right], 334.10(28)\left[\mathrm{M}^{+}\right]$

HRMS (EI): $m / z\left[\mathrm{M}^{+}\right]$calcd for $\mathrm{C}_{21} \mathrm{H}_{20} \mathrm{P}_{2}, 334.1040$; found, 334.1038 .

S4.6 - Acenap(PiPr)(PFc) (6c)<smiles>CPCc1ccc2c3c(ccc(PC(F)(F)F)c13)CC2</smiles>

5c

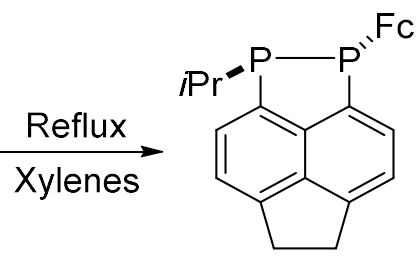

$6 c$

Scheme S9: Synthesis of compound 6 c.

Compound $5 \mathrm{c}(2.81 \mathrm{~g}, 5.79 \mathrm{mmol})$ was dissolved in xylenes $(120 \mathrm{~mL})$ and heated under reflux for 20 hours. Volatiles were removed in vacuo to yield a dark orange viscous oil. Trituration with hexane afforded $6 \mathrm{c}$ as a dark orange solid (2.56 g, $5.79 \mathrm{mmol},>99 \%$ ). 
Crystals suitable for single crystal X-ray diffraction were grown from slow diffusion of ethanol into a concentrated solution of $\mathbf{6 c}$ in DCM at room temperature.

$\mathrm{mp} 147-149^{\circ} \mathrm{C}$.

Anal Calcd for $\mathrm{C}_{25} \mathrm{H}_{24} \mathrm{FeP}_{2}$ : C 67.90; H 5.47. Found: $\mathrm{C}, 67.80 ; \mathrm{H}, 5.56$.

IR (KBr disk) $\bar{v}_{\text {max }}: 3096(\mathrm{~m}), 3038$ (m, CH, aromatic), 2946 (s), 2916 (s, CH, aliphatic), 2857 (s), $1871(\mathrm{w})$, $1656(\mathrm{~m}), 1605(\mathrm{~m}), 1567(\mathrm{~m}), 1446(\mathrm{~m}), 1411(\mathrm{~m}), 1384(\mathrm{~s}), 1356(\mathrm{~m}), 1261(\mathrm{~s}), 1218(\mathrm{~m}), 1159(\mathrm{~s}), 1103$ $(\mathrm{s}), 1025(\mathrm{~s}), 843(\mathrm{~s}), 818(\mathrm{vs}) \mathrm{cm}^{-1}$.

${ }^{1} \mathrm{H} \mathrm{NMR}\left(500 \mathrm{MHz}, \mathrm{CDCl}_{3}, \delta\right): 7.77\left(\mathrm{dd},{ }^{3} \mathrm{~J}_{\mathrm{HH}}=6.9 \mathrm{~Hz},{ }^{3} \mathrm{HP}_{\mathrm{HP}}=5.5 \mathrm{~Hz}, 1 \mathrm{H}, 2-\mathrm{H}\right), 7.68\left(\mathrm{dd},{ }^{3} \int_{\mathrm{HH}}=6.9 \mathrm{~Hz},{ }^{3} \mathrm{JP}_{\mathrm{HP}}=4.9\right.$ $\mathrm{Hz}, 1 \mathrm{H}, 8-\mathrm{H}), 7.37-7.31(\mathrm{~m}, 2 \mathrm{H}, 3-\mathrm{H}, 7-\mathrm{H}), 4.19(\mathrm{~s}, 7 \mathrm{H}, 7 \times \mathrm{CpH}), 4.11(\mathrm{br} \mathrm{s}, 2 \mathrm{H}, 2 \times \mathrm{CpH}), 3.73(\mathrm{br} \mathrm{s}, 4 \mathrm{H}, 11-$ $\mathrm{H}, 12-\mathrm{H}), 1.85\left(\mathrm{dsept},{ }^{2} J_{\mathrm{HP}}=7.0 \mathrm{~Hz},{ }^{3} J_{\mathrm{HH}}=7.0 \mathrm{~Hz}, 1 \mathrm{H}, i \operatorname{PrCH}\right), 1.17\left(\mathrm{dd},{ }^{3} J_{\mathrm{HP}}=14.6,{ }^{3} \mathrm{JHH}_{\mathrm{HH}}=7.0 \mathrm{~Hz}, 3 \mathrm{H}, i \operatorname{Pr} \mathrm{CH}_{3}\right)$, $1.08\left(\mathrm{dd},{ }^{3} \mathrm{JP}_{\mathrm{HP}}=14.1 \mathrm{~Hz},{ }^{3} \mathrm{JHH}_{\mathrm{HH}}=6.9 \mathrm{~Hz}, 3 \mathrm{H}, i \mathrm{Pr} \mathrm{CH}_{3}\right)$.

${ }^{13} \mathrm{C}\left\{{ }^{1} \mathrm{H}\right\}$ NMR $\left(126 \mathrm{MHz}, \mathrm{CDCl}_{3}, \delta\right): 146.03$ (s, qC-4/6) 145.97 (s, qC-4/6), 141.1 (s, qC-10), 139.2 (qC-5) 138.4 $\left(\mathrm{d},{ }^{1} J_{\mathrm{CP}}=23.9 \mathrm{~Hz}, \mathrm{qC}-1\right), 137.3\left(\mathrm{~d},{ }^{1} J_{\mathrm{CP}}=31.0 \mathrm{~Hz}, \mathrm{qC}-9\right) 131.9\left(\mathrm{~d},{ }^{2} \mathrm{~J}_{\mathrm{CP}}=20.1 \mathrm{~Hz}, \mathrm{C}-8\right), 131.3\left(\mathrm{~d},{ }^{2} \mathrm{~J}_{\mathrm{CP}}=21.3 \mathrm{~Hz}\right.$, C-2), $120.4\left(\mathrm{~d},{ }^{3} J_{\mathrm{CP}}=6.6 \mathrm{~Hz}, \mathrm{C}-3 / 7\right), 120.3\left(\mathrm{~d},{ }^{3} \mathrm{JP}_{\mathrm{CP}}=6.5 \mathrm{~Hz}, \mathrm{C}-3 / 7\right), 78.6\left(\approx \mathrm{t},{ }^{1} J_{\mathrm{CP}}=16.1 \mathrm{~Hz},{ }^{2}{ }_{\mathrm{CP}}=16.1 \mathrm{~Hz}, \mathrm{Cp}\right.$ q-C), $72.0(\mathrm{~m}, \mathrm{Cp} \mathrm{CH}), 71.8\left(\mathrm{dd}, J_{\mathrm{CP}}=12.7 \mathrm{~Hz}, J_{\mathrm{CP}}=6.9 \mathrm{~Hz}, \mathrm{Cp} \mathrm{CH}\right), 70.6(\mathrm{br} \mathrm{s}, \mathrm{Cp} \mathrm{CH}), 69.8\left(\mathrm{~d}, J_{\mathrm{CP}}=2.9 \mathrm{~Hz}\right.$, $\mathrm{Cp} \mathrm{CH}), 69.1(\mathrm{~s}, 5 \times \mathrm{Cp} \mathrm{CH}), 30.8\left(\mathrm{~d},{ }^{5} J_{\mathrm{CP}}=2.7 \mathrm{~Hz}, \mathrm{C}-11 / 12\right), 29.8(\mathrm{br} \mathrm{s}, \mathrm{C}-11 / 12), 28.5\left(\approx \mathrm{t},{ }^{1} J_{\mathrm{CP}}=15.4 \mathrm{~Hz},{ }^{2} \mathrm{~J}_{\mathrm{CP}}\right.$ $=15.4 \mathrm{~Hz}, i \mathrm{Pr} \mathrm{CH}), 20.9\left(\mathrm{dd},{ }^{2} \mathrm{~J}_{\mathrm{CP}}=12.7 \mathrm{~Hz},{ }^{3} \mathrm{~J}_{\mathrm{CP}}=10.6 \mathrm{~Hz}, i \operatorname{Pr~CH} 3\right), 20.4\left(\mathrm{dd},{ }^{2} J_{\mathrm{CP}}=12.8 \mathrm{~Hz},{ }^{3} J_{\mathrm{CP}}=7.9 \mathrm{~Hz}, i \mathrm{Pr}\right.$ $\left.\mathrm{CH}_{3}\right)$.

${ }^{31} \mathrm{P}$ NMR $\left(203 \mathrm{MHz}, \mathrm{CDCl}_{3}, \delta\right): 11.9(\mathrm{br} \mathrm{d}, i \mathrm{PrP}),-24.6$ (d, PPh), ${ }^{1}{ }_{\mathrm{PP}}=233 \mathrm{~Hz}$.

${ }^{31} \mathrm{P}\left\{{ }^{1} \mathrm{H}\right\}$ NMR $\left(203 \mathrm{MHz}, \mathrm{CDCl}_{3}, \delta\right): 11.9(\mathrm{~d}, \mathrm{PPrP}),-24.6(\mathrm{~d}, \mathrm{PPh}),{ }^{1}{ }_{\mathrm{PP}}=233 \mathrm{~Hz}$.

MS (EI): $m / z$ (\%) $257.05(18)\left[\mathrm{M}^{+}-\mathrm{Ph}\right], 291.04(100)\left[\mathrm{M}^{+}-i \mathrm{Pr}\right], 334.10(28)\left[\mathrm{M}^{+}\right]$

HRMS (EI): $m / z\left[\mathrm{M}^{+}\right]$calcd for $\mathrm{C}_{25} \mathrm{H}_{24} \mathrm{FeP}_{2}, 442.0703$; found, 442.0699 .

S4.7 - Acenap(PiPr 2$)(\mathrm{As}(\mathrm{Ph}) \mathrm{Cl})(7)$

\section{S4.7.1 - Synthesis of crude 7}

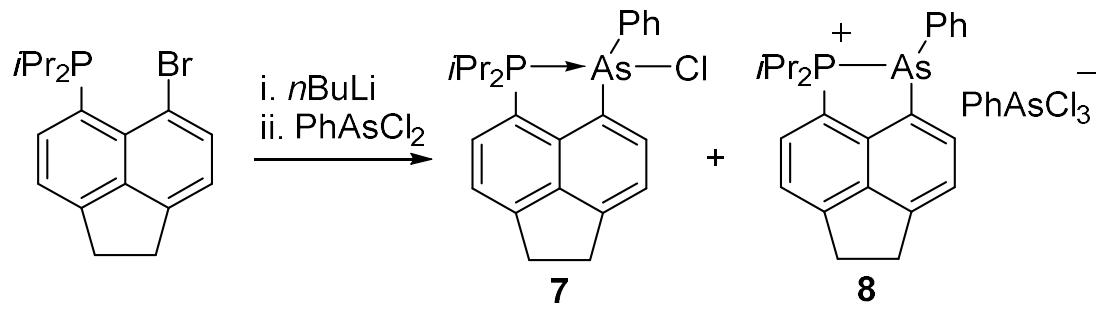

Scheme S10: Synthesis of crude 7, contaminated with compound 8.

To a cooled $\left(-78^{\circ} \mathrm{C}\right)$, rapidly stirring solution of 5-bromo-6-diisopropylphosphinoacenaphthene $(3.00 \mathrm{~g}$, $8.59 \mathrm{mmol})$ in diethyl ether $(120 \mathrm{~mL}), n$ BuLi $(3.4 \mathrm{~mL}$ of a $2.5 \mathrm{M}$ solution in hexanes, $8.6 \mathrm{mmol})$ was added dropwise, and the mixture was left to stir for two hours at $-78^{\circ} \mathrm{C}$. A solution of dichlorophenylarsine $(1.16$ $\mathrm{mL}, 8.59 \mathrm{mmol})$ in diethyl ether $(10 \mathrm{~mL})$ was added swiftly, over a period of a few minutes, to the reaction. 
The reaction mixture was left to stir and warm up to room temperature overnight. The white solid was collected by filtration and washed with diethyl ether $(2 \times 20 \mathrm{~mL})$. The solid was dried in vacuo to give the product $(7)$ as a white powder $(3.16 \mathrm{~g})$, which was contaminated with compound $\mathbf{8}\left(\approx 30 \%\right.$ by $\left.{ }^{1} \mathrm{H} \mathrm{NMR}\right)$.

Crystals suitable for X-ray diffraction were obtained from acetonitrile at $0{ }^{\circ} \mathrm{C}$. Analytically pure $\mathbf{7}$ was obtained by the reaction of 9 with chloroform (see below)

\section{S4.7.2 - Synthesis of pure 7}

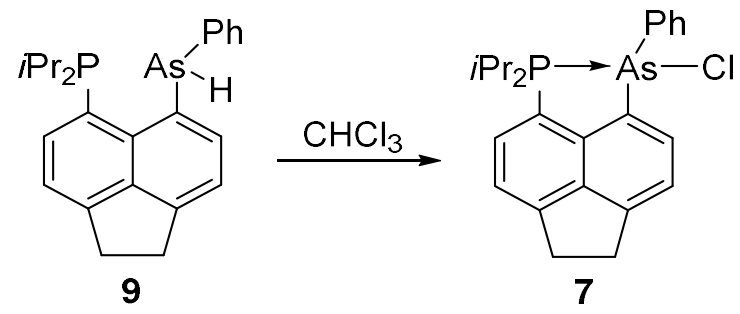

Scheme S11: Synthesis of pure $\mathbf{7}$ by the chlorination of 9 with chloroform.

Compound $9(1.30 \mathrm{~g}, 3.08 \mathrm{mmol})$ was dissolved in chloroform $(10 \mathrm{~mL})$ to give a yellow solution, which gradually produced a white precipitate. After stirring the reaction overnight, diethyl ether $(20 \mathrm{~mL})$ was added. The white precipitate was collected by filtration and washed with diethyl ether $(10 \mathrm{~mL})$. The solid was dried in vacuo to afford analytically pure 7 as a fine white powder (1.048 g, $2.29 \mathrm{mmol}, 74 \%$ ).

Anal Calcd for $\mathrm{C}_{24} \mathrm{H}_{27} \mathrm{PAsCl}$ : C, 63.10; $\mathrm{H}, 5.96$. Found: $\mathrm{C}, 62.92 ; \mathrm{H}, 6.03$.

${ }^{1} \mathrm{H} \mathrm{NMR}\left(400 \mathrm{MHz}, \mathrm{CDCl}_{3}, \delta\right): 8.56\left(\mathrm{dd},{ }^{3} \mathrm{~J}_{\mathrm{HP}}=9.0 \mathrm{~Hz},{ }^{3} \mathrm{~J}_{\mathrm{HH}}=7.2 \mathrm{~Hz}, 1 \mathrm{H}, 8-\mathrm{H}\right), 8.00\left(\mathrm{~d},{ }^{3} \mathrm{JHH}_{\mathrm{HH}}=7.1 \mathrm{~Hz}, 1 \mathrm{H}, 2-\mathrm{H}\right)$, $7.68\left(\mathrm{dd},{ }^{3} J_{\mathrm{HH}}=7.2 \mathrm{~Hz},{ }^{4} J_{\mathrm{HP}}=2.3 \mathrm{~Hz}, 1 \mathrm{H}, 7-\mathrm{H}\right), 7.60\left(\mathrm{~d},{ }^{3} \mathrm{H}_{\mathrm{HH}}=7.1 \mathrm{~Hz}, 1 \mathrm{H}, 3-\mathrm{H}\right), 7.42-7.38(\mathrm{~m}, 2 \mathrm{H}, o-\mathrm{Ph} \mathrm{CH})$, 7.38-7.34 (m, 1H, p-Ph CH), 7.32-7.27 (m, 2H, m-Ph CH), 4.02-3.89 (m, $1 \mathrm{H}, i \operatorname{Pr~CH}), 3.89-3.75(\mathrm{~m}, 1 \mathrm{H}, i \mathrm{Pr}$ $\mathrm{CH}), 3.59(\mathrm{~s}, 4 \mathrm{H}, 11-\mathrm{H}, 12-\mathrm{H}), 1.37\left(\mathrm{dd},{ }^{3} \mathrm{HP}_{\mathrm{HP}}=19.9 \mathrm{~Hz},{ }^{3} J_{\mathrm{HH}}=6.9 \mathrm{~Hz}, 3 \mathrm{H}, i \mathrm{PrCH}_{3}\right), 1.20\left(\mathrm{dd},{ }^{3} J_{\mathrm{HP}}=18.2 \mathrm{~Hz},{ }^{3} J_{\mathrm{HH}}\right.$ $\left.=7.0 \mathrm{~Hz}, 3 \mathrm{H}, i \mathrm{Pr} \mathrm{CH}_{3}\right), 1.04-0.93\left(\mathrm{~m}, 6 \mathrm{H}, 2 \times i \mathrm{PrCH}_{3}\right)$.

${ }^{13} \mathrm{C}\left\{{ }^{1} \mathrm{H}\right\} \mathrm{NMR}\left(101 \mathrm{MHz}, \mathrm{CDCl}_{3}, \delta\right): 153.9\left(\mathrm{~d},{ }^{4} \mathrm{~J}_{\mathrm{CP}}=2.7 \mathrm{~Hz}, \mathrm{qC}-6\right), 149.0(\mathrm{~s}, \mathrm{qC}-4), 142.3\left(\mathrm{~d},{ }^{2} \mathrm{~J}_{\mathrm{CP}}=21.8 \mathrm{~Hz}, \mathrm{qC}-\right.$ 10), $139.7\left(\mathrm{~d},{ }^{3} \mathrm{~J}_{\mathrm{CP}}=12.2 \mathrm{~Hz}, \mathrm{qC}-5\right), 136.8(\mathrm{~s}, \mathrm{C}-8), 135.1\left(\mathrm{~d},{ }^{3} \mathrm{~J}_{\mathrm{CP}}=6.9 \mathrm{~Hz}, \mathrm{C}-2\right), 134.5\left(\mathrm{~d},{ }^{3} \mathrm{JPP}_{\mathrm{CP}}=3.9 \mathrm{~Hz}, o-\mathrm{Ph}\right.$ $\mathrm{CH}), 130.9\left(\mathrm{~d},{ }^{5} \mathrm{~J}_{\mathrm{CP}}=3.0 \mathrm{~Hz}, p-\mathrm{Ph} \mathrm{CH}\right), 130.8\left(\mathrm{~d},{ }^{2} J_{\mathrm{CP}}=3.0 \mathrm{~Hz}, \mathrm{qC}-1\right), 130.1\left(\mathrm{~d},{ }^{2} J_{\mathrm{CP}}=8.5 \mathrm{~Hz}, i-\mathrm{Ph} \mathrm{qC}\right), 129.7$ (d, $\left.{ }^{4} J_{\mathrm{CP}}=2.4 \mathrm{~Hz}, m-\mathrm{Ph} \mathrm{CH}\right), 122.3(\mathrm{~s}, \mathrm{C}-7), 122.2(\mathrm{~s}, \mathrm{C}-3), 115.4$ (d, $\left.{ }^{1} \mathrm{JP}_{\mathrm{CP}}=47.9 \mathrm{~Hz}, \mathrm{qC}-1\right), 31.4$ (s, C-11/C-12), 30.9 (s, C-11/C-12), 26.8 (d, $\left.{ }^{1} J_{\mathrm{CP}}=22.1 \mathrm{~Hz}, i \operatorname{Pr~CH}\right), 26.2\left(\mathrm{~d},{ }^{1} \mathrm{~J}_{\mathrm{CP}}=30.4 \mathrm{~Hz}, \mathrm{PPr} \mathrm{CH}\right.$ ), 18.6 (s, $\left.i \operatorname{Pr~CH}_{3}\right), 18.1$ (d, $\left.{ }^{2} J_{\mathrm{CP}}=3.6 \mathrm{~Hz}, i \operatorname{PrCH} \mathrm{CH}_{3}\right), 17.83\left(\mathrm{~d},{ }^{2} J_{\mathrm{CP}}=1.5 \mathrm{~Hz}, i \operatorname{Pr} \mathrm{CH}_{3}\right), 17.75\left(\mathrm{~d},{ }^{2} J_{\mathrm{CP}}=4.5 \mathrm{~Hz}, i \operatorname{Pr} \mathrm{CH}_{3}\right)$.

${ }^{31} \mathrm{P}$ NMR $\left(162 \mathrm{MHz}, \mathrm{CDCl}_{3}, \delta\right): 58.8(\mathrm{~m})$.

${ }^{31} \mathrm{P}\left\{{ }^{1} \mathrm{H}\right\} \mathrm{NMR}\left(162 \mathrm{MHz}, \mathrm{CDCl}_{3}, \delta\right): 58.8(\mathrm{~s})$.

MS (ES+): $m / z(\%) 421.11(100)\left[\mathrm{M}^{+}-\mathrm{Cl}\right]$

HRMS (ES+): $m / z\left[\mathrm{M}^{+}-\mathrm{Cl}\right]$ calcd for $\mathrm{C}_{24} \mathrm{H}_{27} \mathrm{PAs}, 421.1066$; found, 421.1050 . 
S4.8 - $\left[\mathrm{Acenap}\left(\mathrm{PiPr}_{2}\right)(\mathrm{AsPh})\right]\left[\mathrm{PhAsCl}_{3}\right](\mathbf{8})$

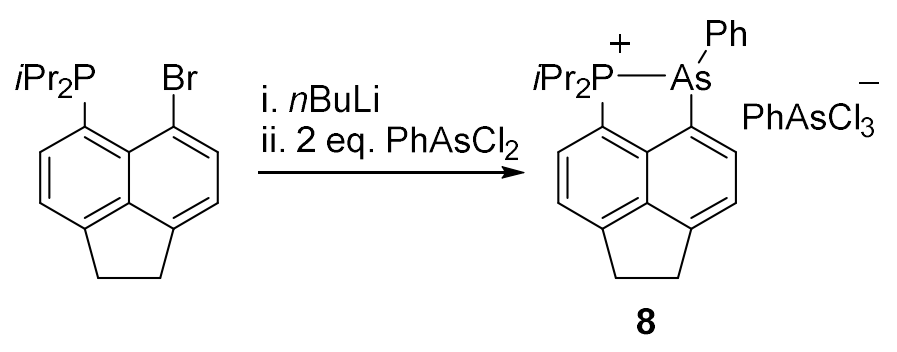

Scheme S12: Synthesis of pure compound 8.

To a cooled $\left(-78^{\circ} \mathrm{C}\right)$, rapidly stirring solution of 5-bromo-6-diisopropylphosphinoacenaphthene $(2.00 \mathrm{~g}$, $5.73 \mathrm{mmol}$ ) in diethyl ether $(50 \mathrm{~mL}), n B u L i(2.30 \mathrm{~mL}$ of a $2.5 \mathrm{M}$ solution in hexanes, $5.73 \mathrm{mmol})$ was added dropwise over $1 \mathrm{~h}$, and the mixture was left to stir for $2 \mathrm{~h}$ at $-78^{\circ} \mathrm{C}$. A solution of dichlorophenylarsine $(2.55 \mathrm{~g}, 1.55 \mathrm{~mL}, 11.46 \mathrm{mmol})$ in diethyl ether $(15 \mathrm{~mL})$ was then added dropwise over $1 \mathrm{~h}$ at $-78^{\circ} \mathrm{C}$. The reaction mixture was left to stir and warm up to room temperature overnight. The white precipitate was collected by filtration and washed with diethyl ether $(20 \mathrm{~mL})$. The solid was dried in vacuo to give compound 8 as a white powder ( $2.33 \mathrm{~g}, 3.43 \mathrm{mmol}, 60 \%)$. Crystals suitable for X-ray diffraction were grown from acetonitrile at $0^{\circ} \mathrm{C}$.

Anal Calcd for $\mathrm{C}_{30} \mathrm{H}_{32} \mathrm{PAs}_{2} \mathrm{Cl}_{3}: \mathrm{C}, 53.01 ; \mathrm{H}, 4.75$. Found: $\mathrm{C}, 52.92 ; \mathrm{H}, 4.80$.

${ }^{1} \mathrm{H} \mathrm{NMR}\left(500 \mathrm{MHz}, \mathrm{CDCl}_{3}, \delta\right): 8.53\left(\mathrm{dd},{ }^{3} \mathrm{~J}_{\mathrm{HP}}=9.1 \mathrm{~Hz},{ }^{3} \mathrm{~J}_{\mathrm{HH}}=7.2 \mathrm{~Hz}, 1 \mathrm{H}, 8-\mathrm{H}\right), 8.00\left(\mathrm{~d},{ }^{3} \mathrm{~J}_{\mathrm{HH}}=7.0 \mathrm{~Hz}, 1 \mathrm{H}, 2-\mathrm{H}\right)$,

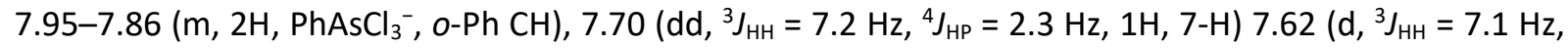
$1 \mathrm{H}, 3-\mathrm{H}), 7.58-7.49\left(\mathrm{~m}, 3 \mathrm{H}, \mathrm{PhAsCl}_{3}{ }^{-}, \mathrm{m} / \mathrm{p}-\mathrm{Ph} \mathrm{CH}\right), 7.44-7.35(\mathrm{~m}, 3 \mathrm{H}, o / p-\mathrm{Ph} \mathrm{CH}), 7.34-7.29(\mathrm{~m}, 2 \mathrm{H}, m-\mathrm{Ph}$ $\mathrm{CH}$ ), 3.88 (dsept, ${ }^{2} J_{\mathrm{HP}}=10.3 \mathrm{~Hz},{ }^{3} \jmath_{\mathrm{HH}}=7.0 \mathrm{~Hz}, 1 \mathrm{H}, i \mathrm{Pr} \mathrm{CH}$ ), 3.73 (dsept, ${ }^{2} J_{\mathrm{HP}}=14.1 \mathrm{~Hz},{ }^{3} J_{\mathrm{HH}}=7.1 \mathrm{~Hz}, 1 \mathrm{H}, i \mathrm{Pr}$ $\mathrm{CH}), 3.66-3.56(\mathrm{~m}, 4 \mathrm{H}, 11-\mathrm{H}, 12-\mathrm{H}), 1.37\left(\mathrm{dd},{ }^{3} J_{\mathrm{HP}}=19.9 \mathrm{~Hz},{ }^{3} J_{\mathrm{HH}}=6.8 \mathrm{~Hz}, 3 \mathrm{H}, i \operatorname{Pr} \mathrm{CH}_{3}\right), 1.22\left(\mathrm{dd},{ }^{3} J_{\mathrm{HP}}=18.2\right.$ $\mathrm{Hz},{ }^{3} J_{\mathrm{HH}}=6.9 \mathrm{~Hz}, 3 \mathrm{H}, i \mathrm{Pr} \mathrm{CH}$ ) $, 1.04\left(\mathrm{dd},{ }^{3} J_{\mathrm{HP}}=19.5 \mathrm{~Hz},{ }^{3} J_{\mathrm{HH}}=7.0 \mathrm{~Hz}, 3 \mathrm{H}, i \operatorname{Pr} \mathrm{CH}_{3}\right), 0.97\left(\mathrm{dd},{ }^{3} \mathrm{JPP}_{\mathrm{HP}}=19.1 \mathrm{~Hz}\right.$, $\left.{ }^{3} J_{\mathrm{HH}}=7.1 \mathrm{~Hz}, 3 \mathrm{H}, i \mathrm{Pr} \mathrm{CH}_{3}\right)$.

${ }^{13} \mathrm{C}\left\{{ }^{1} \mathrm{H}\right\}$ NMR $\left(126 \mathrm{MHz}, \mathrm{CDCl}_{3}, \delta\right): 153.8\left(\mathrm{~d},{ }^{4} \mathrm{~J}_{\mathrm{CP}}=2.9 \mathrm{~Hz}, \mathrm{qC}-6\right), 148.9(\mathrm{~s}, \mathrm{qC}-4), 142.2\left(\mathrm{~d},{ }^{2} J_{\mathrm{CP}}=21.5 \mathrm{~Hz}, \mathrm{qC}-\right.$ 10), $139.7\left(\mathrm{~d},{ }^{3} \mathrm{~J}_{\mathrm{CP}}=12.0 \mathrm{~Hz}, \mathrm{qC}-5\right), 136.9(\mathrm{~s}, \mathrm{C}-8), 134.9\left(\mathrm{~d},{ }^{3} \mathrm{~J}_{\mathrm{CP}}=7.0 \mathrm{~Hz}, \mathrm{C}-2\right), 134.4\left(\mathrm{~d},{ }^{3} \mathrm{JP}_{\mathrm{CP}}=3.8 \mathrm{~Hz}, o-\mathrm{Ph}\right.$ $\mathrm{CH}), 131.9$ (s, PhAsCl $\left.{ }^{-}, p-\mathrm{Ph} \mathrm{CH}\right), 130.9$ (d, ${ }^{4} J_{\mathrm{CP}}=3.0 \mathrm{~Hz}, \mathrm{~m}$-Ph CH), 130.7 (s, qC-1), 130.6 (s, i-Ph qC), 130.0 (s, $\left.\mathrm{PhAsCl}_{3}{ }^{-}, o-\mathrm{Ph} \mathrm{CH}\right), 129.9$ (s, $\left.\mathrm{PhAsCl}_{3}{ }^{-}, i-\mathrm{Ph} \mathrm{qC}\right), 129.6$ (d, $\left.{ }^{5} \mathrm{CP}_{\mathrm{CP}}=2.3 \mathrm{~Hz}, p-\mathrm{Ph} \mathrm{CH}\right), 129.1\left(\mathrm{~s}, \mathrm{PhAsCl}_{3}{ }^{-}, m-\right.$ $\mathrm{Ph} \mathrm{CH}), 122.3\left(\mathrm{~d},{ }^{3} \mathrm{JP}_{\mathrm{CP}}=9.9 \mathrm{~Hz}, \mathrm{C}-3\right), 122.1(\mathrm{~s}, \mathrm{C}-7), 115.3\left(\mathrm{~d},{ }^{1} \mathrm{~J}_{\mathrm{CP}}=48.3 \mathrm{~Hz}, \mathrm{qC}-9\right), 31.3(\mathrm{~s}, \mathrm{C}-11 / \mathrm{C}-12), 30.8$ (s, C-11/C-12), $26.8\left(\mathrm{~d},{ }^{1} J_{\mathrm{CP}}=22.1 \mathrm{~Hz}, i \operatorname{Pr} \mathrm{CH}\right), 26.2\left(\mathrm{~d},{ }^{1} \mathrm{~J}_{\mathrm{CP}}=30.4 \mathrm{~Hz}, i \operatorname{PrCH}\right), 18.6\left(\mathrm{~s}, i \operatorname{PrCH} \mathrm{CH}_{3}\right), 18.1\left(\mathrm{~d},{ }^{2} J_{\mathrm{CP}}=\right.$ 3.6 Hz, $\left.i \operatorname{Pr~} \mathrm{CH}_{3}\right), 17.8\left(\mathrm{~s}, i \operatorname{Pr} \mathrm{CH}_{3}\right), 17.7\left(\mathrm{~d},{ }^{2} \mathrm{~J}_{\mathrm{CP}}=4.5 \mathrm{~Hz}, i \operatorname{Pr} \mathrm{CH}_{3}\right)$.

${ }^{31} \mathrm{P}\left\{{ }^{1} \mathrm{H}\right\} \mathrm{NMR}\left(203 \mathrm{MHz}, \mathrm{CDCl}_{3}, \delta\right): 59.4(\mathrm{~s})$.

MS (ES+): $m / z(\%) 421.10(100)\left[\mathrm{M}^{+}-\mathrm{PhAsCl}_{3}\right]$

HRMS (ES+): $m / z\left[\mathrm{M}^{+}-\mathrm{PhAsCl}_{3}\right]$ calcd for $\mathrm{C}_{24} \mathrm{H}_{27} \mathrm{PAs}, 421.1066$; found, 421.1045 . 
S4.9 - Acenap( $\left(\operatorname{PiPr}_{2}\right)(\mathrm{As}(\mathrm{Ph}) \mathrm{H})(9)$

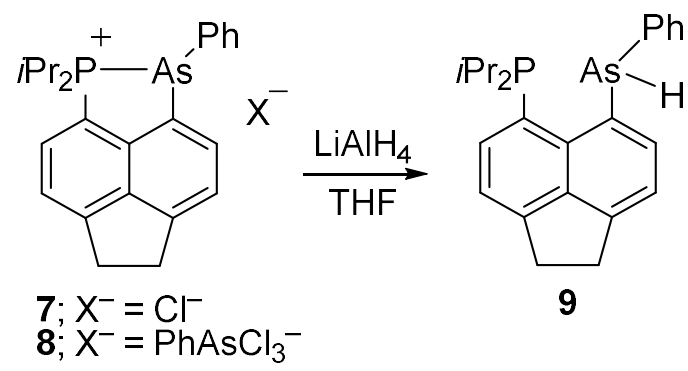

Scheme S13: Synthesis of compound 9.

To a cooled $\left(0^{\circ} \mathrm{C}\right)$ stirred suspension of crude $7(4.28 \mathrm{~g})$ in THF $(100 \mathrm{~mL})$, a suspension of $\mathrm{LiAlH}_{4}(0.39 \mathrm{~g}, 10$ $\mathrm{mmol})$ in THF $(20 \mathrm{~mL}$ ) was added in small portions over 30 minutes. The reaction was left to stir at room temperature for 4 hours. Degassed water $(1 \mathrm{~mL})$ was added cautiously to neutralise the excess $\mathrm{LiAlH}_{4}$. The solution was filtered and the volatiles removed in vacuo. Hexane $(100 \mathrm{~mL})$ was added and the mixture filtered to remove the insoluble by-products. Volatiles were removed in vacuo to afford 9 as a pale yellow powder ( $2.98 \mathrm{~g}, 7.05 \mathrm{mmol}, 61 \%$ yield based on 5-bromo-6-diisopropylphosphinoacenaphthene).

${ }^{1} \mathrm{H}$ NMR $\left(500 \mathrm{MHz}, \mathrm{C}_{6} \mathrm{D}_{6}, \delta\right): 8.13\left(\mathrm{~d},{ }^{3} \mathrm{~J}_{\mathrm{HH}}=7.1 \mathrm{~Hz}, 1 \mathrm{H}, 2-\mathrm{H}\right), 7.70-7.67(\mathrm{~m}, 2 \mathrm{H}, \mathrm{o}-\mathrm{Ph} \mathrm{CH}), 7.62\left(\mathrm{dd},{ }^{3} \mathrm{~J}_{\mathrm{HH}}=7.2\right.$ $\left.\mathrm{Hz},{ }^{3} J_{\mathrm{HP}}=3.3 \mathrm{~Hz}, 1 \mathrm{H}, 8-\mathrm{H}\right), 7.19\left(\mathrm{~d},{ }^{3} J_{\mathrm{HH}}=7.2 \mathrm{~Hz}, 1 \mathrm{H}, 7-\mathrm{H}\right), 7.16-7.11(\mathrm{~m}, 3 \mathrm{H}, \mathrm{m} / \mathrm{p}-\mathrm{Ph} \mathrm{CH}), 7.08\left(\mathrm{~d},{ }^{3} \mathrm{JHH}_{\mathrm{HH}}=7.1\right.$ $\mathrm{Hz}, 1 \mathrm{H}, 3-\mathrm{H}), 6.02\left(\mathrm{~d},{ }^{5} J_{\mathrm{HP}}=80.4 \mathrm{~Hz}, 1 \mathrm{H}, \mathrm{AsH}\right), 3.06-3.00(\mathrm{~m}, 4 \mathrm{H}, 11-\mathrm{H}, 12-\mathrm{H}), 2.03(\mathrm{~m}, 1 \mathrm{H}, i \mathrm{Pr} \mathrm{CH}), 1.26$ (dd, $\left.{ }^{3} J_{\mathrm{HP}}=14.0 \mathrm{~Hz},{ }^{3} J_{\mathrm{HH}}=7.0 \mathrm{~Hz}, 3 \mathrm{H}, i \operatorname{PrCH} \mathrm{CH}_{3}\right), 1.16$ (dsept, $\left.{ }^{2} J_{\mathrm{HP}}=14.1 \mathrm{~Hz},{ }^{3} J_{\mathrm{HH}}=7.1 \mathrm{~Hz}, 1 \mathrm{H}, i \operatorname{PrCH}\right), 1.11\left(\mathrm{dd},{ }^{3} \mathrm{JPP}_{\mathrm{HP}}\right.$ $\left.=8.8 \mathrm{~Hz},{ }^{3} \mathrm{~J}_{\mathrm{HH}}=6.9 \mathrm{~Hz}, 3 \mathrm{H}, i \operatorname{Pr} \mathrm{CH}_{3}\right), 1.09\left(\mathrm{dd},{ }^{3} \mathrm{~J}_{\mathrm{HP}}=13.5 \mathrm{~Hz},{ }^{3} \mathrm{JHH}_{\mathrm{HH}}=6.9 \mathrm{~Hz}, 3 \mathrm{H}, i \operatorname{Pr} \mathrm{CH}_{3}\right), 0.54\left(\mathrm{dd},{ }^{3} \mathrm{JP}_{\mathrm{HP}}=13.2\right.$ $\left.\mathrm{Hz},{ }^{3} \mathrm{~J}_{\mathrm{HH}}=7.0 \mathrm{~Hz}, 3 \mathrm{H}, i \mathrm{Pr} \mathrm{CH}_{3}\right)$.

${ }^{13} \mathrm{C}\left\{{ }^{1} \mathrm{H}\right\}$ NMR (126 MHz, $\left.\mathrm{C}_{6} \mathrm{D}_{6}, \delta\right): 148.7$ (s, qC-6), 147.7 (s, qC-4), 142.2 (s, C-2), 140.4 (d, ${ }^{3} \mathrm{~J}_{\mathrm{CP}}=3.7 \mathrm{~Hz}, \mathrm{qC}-$ 5), $140.3\left(\mathrm{~d},{ }^{2} J_{\mathrm{CP}}=4.1 \mathrm{~Hz}, \mathrm{qC}-10\right), 140.2\left(\mathrm{~d},{ }^{5} \mathrm{~J}_{\mathrm{CP}}=5.5 \mathrm{~Hz}, i-\mathrm{Ph} \mathrm{qC}\right), 134.8\left(\mathrm{~d},{ }^{6} J_{\mathrm{CP}}=1.8 \mathrm{~Hz}, o-\mathrm{Ph} \mathrm{CH}\right), 134.3$ (d, $\left.{ }^{2} J_{\mathrm{CP}}=2.4 \mathrm{~Hz}, \mathrm{C}-8\right), 132.4(\mathrm{~s}, \mathrm{qC}-1), 130.1\left(\mathrm{~d},{ }^{1} \mathrm{~J}_{\mathrm{CP}}=22.9 \mathrm{~Hz}, \mathrm{qC}-9\right), 128.1(\mathrm{~s}, m-\mathrm{Ph} \mathrm{CH}), 127.3(\mathrm{~s}, p-\mathrm{Ph} \mathrm{CH})$, 120.1 (s, C-3), 119.1 (s, C-7), 30.0 (s, C-11/C-12), 29.7 (s, C-11/C-12), 26.8 (d, ${ }^{1} J_{\mathrm{CP}}=15.0 \mathrm{~Hz}, i \mathrm{Pr}$ CH), 25.2 $\left(\mathrm{d},{ }^{1} J_{\mathrm{CP}}=16.9 \mathrm{~Hz}, i \operatorname{PrCH}\right), 20.2\left(\mathrm{~d},{ }^{2} J_{\mathrm{CP}}=15.4 \mathrm{~Hz}, i \operatorname{Pr} \mathrm{CH}_{3}\right), 20.0\left(\mathrm{~d},{ }^{2} \mathrm{~J}_{\mathrm{CP}}=22.4 \mathrm{~Hz}, i \operatorname{Pr} \mathrm{CH}_{3}\right), 19.4\left(\mathrm{~s}, i \operatorname{Pr} \mathrm{CH}_{3}\right)$, $19.3\left(\mathrm{~d},{ }^{2} \mathrm{JP}_{\mathrm{CP}}=8.2 \mathrm{~Hz}, i \mathrm{Pr} \mathrm{CH}_{3}\right)$.

${ }^{31}$ P NMR $\left(203 \mathrm{MHz}, \mathrm{C}_{6} \mathrm{D}_{6}, \delta\right):-13.1\left(\mathrm{~d},{ }^{5} \mathrm{JPH}_{\mathrm{PH}}=80.4 \mathrm{~Hz}\right)$.

${ }^{31} \mathrm{P}\left\{{ }^{1} \mathrm{H}\right\}$ NMR (203 MHz, $\left.\mathrm{C}_{6} \mathrm{D}_{6}, \delta\right):-13.1(\mathrm{~s})$.

MS (ES+): $m / z(\%) 423.12(100)\left[\mathrm{M}+\mathrm{H}^{+}\right]$

HRMS (APCl+): $m / z\left[M+\mathrm{H}^{+}\right]$calcd for $\mathrm{C}_{24} \mathrm{H}_{29} \mathrm{PAs}, 423.1217$; found, 423.1210 . 
S4.10 - Acenap(PiPr)(AsPh) (10)

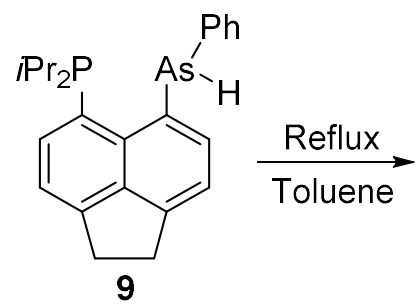

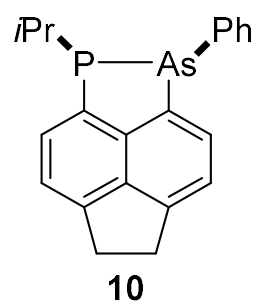

Scheme S14: Synthesis of compound 10

A solution of 9 ( $3.00 \mathrm{~g}, 7.10 \mathrm{mmol})$ was dissolved in toluene $(100 \mathrm{~mL})$ and heated under reflux for 3 hours. The volatiles were removed in vacuo to give a yellow oil which solidified overnight $(2.67 \mathrm{~g}, 7.06 \mathrm{mmol}$, $>99 \%$ ). Crystals suitable for X-ray diffraction were grown by slow diffusion of methanol into a concentrated solution of 10 in DCM at $-35^{\circ} \mathrm{C}$.

$\mathrm{mp} 87-88^{\circ} \mathrm{C}$.

Anal Calcd for $\mathrm{C}_{30} \mathrm{H}_{32} \mathrm{PAs}_{2} \mathrm{Cl}_{3}: \mathrm{C}, 66.68 ; \mathrm{H}, 5.33$. Found: $\mathrm{C}, 66.50 ; \mathrm{H}, 5.26$.

IR (KBr disc) $\bar{\nu}_{\text {max }}: 3041$ (m, CH, aromatic), 2943 (vs, CH, aliphatic), 1865 (m), 1599 (s), 1479 (s), 1377 (vs), $1214(\mathrm{~m}), 1024(\mathrm{~m}), 842(\mathrm{~m}), 733(\mathrm{vs}), 691(\mathrm{~m}), 614(\mathrm{~m}), 585(\mathrm{~m}), 461(\mathrm{w}), 402(\mathrm{w}, \mathrm{PAs}) \mathrm{cm}^{-1}$.

${ }^{1} \mathrm{H} \mathrm{NMR}\left(500 \mathrm{MHz}, \mathrm{CDCl}_{3}, \delta\right): 7.90\left(\mathrm{~d},{ }^{3} \mathrm{~J}_{\mathrm{HH}}=6.9 \mathrm{~Hz}, 1 \mathrm{H}, 2-\mathrm{H}\right), 7.76\left(\approx \mathrm{t},{ }^{3} J_{\mathrm{HP}}=6.6 \mathrm{~Hz},{ }^{3} J_{\mathrm{HH}}=6.6 \mathrm{~Hz}, 1 \mathrm{H}, 8-\mathrm{H}\right)$, $7.45\left(\mathrm{~d},{ }^{3} \mathrm{~J}_{\mathrm{HH}}=7.0 \mathrm{~Hz}, 1 \mathrm{H}, 3-\mathrm{H}\right), 7.40\left(\mathrm{~d},{ }^{3} \mathrm{~J}_{\mathrm{HH}}=7.0 \mathrm{~Hz}, 1 \mathrm{H}, 7-\mathrm{H}\right), 7.35(\mathrm{br} \mathrm{s}, 2 \mathrm{H}, o-\mathrm{Ph} \mathrm{CH}), 7.22(\mathrm{br} \mathrm{s}, 3 \mathrm{H}, \mathrm{m} / \mathrm{p}-$ $\mathrm{Ph} \mathrm{CH}), 3.49(\mathrm{~s}, 4 \mathrm{H}, 11-\mathrm{H}, 12-\mathrm{H}), 2.16-2.02(\mathrm{~m}, 1 \mathrm{H}, \mathrm{Pr} \mathrm{CH}), 1.28\left(\mathrm{dd}, 3_{\mathrm{HP}}=21.6 \mathrm{~Hz},{ }^{3} \mathrm{JHH}_{\mathrm{HH}}=6.9 \mathrm{~Hz}, 3 \mathrm{H}, i \mathrm{Pr}\right.$ $\left.\mathrm{CH}_{3}\right), 1.23\left(\mathrm{dd},{ }^{3} \mathrm{JHP}_{\mathrm{HP}}=21.5 \mathrm{~Hz},{ }^{3} \mathrm{JHH}_{\mathrm{HH}}=6.9 \mathrm{~Hz}, 3 \mathrm{H}, i \mathrm{Pr} \mathrm{CH}_{3}\right)$.

${ }^{13} \mathrm{C}\left\{{ }^{1} \mathrm{H}\right\} \operatorname{NMR}\left(126 \mathrm{MHz}, \mathrm{CDCl}_{3}, \delta\right): 146.4$ (s, qC-6), 146.3 (s, qC-4), 142.4 (s, qC-10), 140.2 (d, ${ }^{3} \mathrm{sP}_{\mathrm{CP}}=9.3 \mathrm{~Hz}$, qC-5), 140.1 (s, i-Ph qC), 139.5 (s, qC-1), 138.2 (d, $\left.{ }^{1} J_{\mathrm{CP}}=34.9 \mathrm{~Hz}, \mathrm{qC}-9\right), 133.1\left(\mathrm{~d},{ }^{2} \mathrm{~J}_{\mathrm{CP}}=21.6 \mathrm{~Hz}, \mathrm{C}-8\right), 132.6$ (s, C-2), $132.4\left(\mathrm{~d},{ }^{3} \mathrm{JP}_{\mathrm{CP}}=5.5 \mathrm{~Hz}, o-\mathrm{Ph} \mathrm{CH}\right), 128.5(\mathrm{~s}, \mathrm{~m}-\mathrm{Ph} \mathrm{CH}), 127.9$ (s, $p$-Ph CH), $120.6(\mathrm{~s}, \mathrm{C}-3), 120.4\left(\mathrm{~d},{ }^{3} \mathrm{~J}_{\mathrm{CP}}\right.$ $=6.9 \mathrm{~Hz}, \mathrm{C}-7), 30.8(\mathrm{~s}, \mathrm{C}-11 / \mathrm{C}-12), 30.6(\mathrm{~s}, \mathrm{C}-11 / \mathrm{C}-12), 28.7\left(\mathrm{~d},{ }^{1} \mathrm{~J}_{\mathrm{CP}}=19.6 \mathrm{~Hz}, i \mathrm{Pr} \mathrm{CH}\right), 21.6\left(\mathrm{~d},{ }^{2} \mathrm{~J}_{\mathrm{CP}}=12.7 \mathrm{~Hz}\right.$, $\left.i \operatorname{Pr} \mathrm{CH}_{3}\right), 21.0\left(\mathrm{~d},{ }^{2} \mathrm{~J}_{\mathrm{CP}}=13.2 \mathrm{~Hz}, i \operatorname{Pr} \mathrm{CH}_{3}\right)$.

${ }^{31} \mathrm{P}\left\{{ }^{1} \mathrm{H}\right\}$ NMR (203 MHz, $\left.\mathrm{CDCl}_{3}, \delta\right): 23.5$ (s).

MS (APCl+): $m / z(\%) 379.06(100)\left[\mathrm{M}+\mathrm{H}^{+}\right], 395.05(65)\left[\mathrm{M}+\mathrm{O}+\mathrm{H}^{+}\right], 411.05(35)\left[\mathrm{M}+20+\mathrm{H}^{+}\right]$.

HRMS (APCl+): $m / z\left[M+\mathrm{H}^{+}\right]$calcd for $\mathrm{C}_{21} \mathrm{H}_{21}$ PAs, 379.0591; found, 379.0581 . 


\section{S5 - Spin Simulation of compound 2}

NMR spectra were obtained on a Bruker Avance III 500 spectrometer at $25^{\circ} \mathrm{C}$. $\mathrm{C}_{6} \mathrm{D}_{6}$ was used as the solvent for these experiments. $85 \% \mathrm{H}_{3} \mathrm{PO}_{4}$ was used as an external standard in ${ }^{31} \mathrm{P} ;{ }^{1} \mathrm{H}$ NMR were referenced to the solvent residual signal. Simulation of spin systems was carried out using the Daisy module in Bruker TopSpin v3.5 pl5. Signs of coupling constants were chosen following reference data. ${ }^{10}$

Compound 2 displays complex second order multiplets in both its ${ }^{1} \mathrm{H}$ and ${ }^{31} \mathrm{P}$ NMR spectra, as expected for an $\mathrm{AA}^{\prime} \mathrm{BB}^{\prime} \mathrm{CC}^{\prime} \mathrm{DD} \mathrm{D}^{\prime} \mathrm{XX}$ ' spin system (Figure S2). Table $\mathrm{S} 3$ gives all relevant ${ }^{31} \mathrm{P}$ and ${ }^{1} \mathrm{H}$ coupling constants, as determined by spin simulation, while Figures $\mathrm{S} 3-\mathrm{S} 5$ show comparisons between the experimentally determined and simulated NMR spectra.

Table S3: Coupling constants $(\mathrm{Hz})$ for compound 2

\begin{tabular}{lccc}
\hline$J_{\mathrm{XX}}$ & -163.4 & $J_{\mathrm{X} D^{\prime}}$ & 17.8 \\
$J_{\mathrm{XA}}$ & -4.8 & $J_{\mathrm{AB}}$ & 7.0 \\
$J_{\mathrm{XB}}$ & 0.7 & $J_{\mathrm{AC}}$ & 1.4 \\
$J_{\mathrm{XC}}$ & -1.2 & $J_{\mathrm{AD}}$ & 2.5 \\
$J_{\mathrm{XD}}$ & 193.44 & $J_{\mathrm{BC}}$ & 8.1 \\
& & $J_{\mathrm{DD}^{\prime}}$ & 16.0 \\
\hline
\end{tabular}

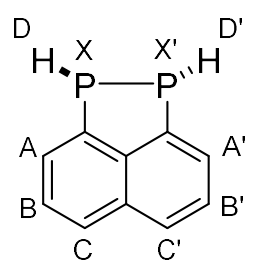<smiles>c1cc2cccc3cccc(c1)-p-3-p-2</smiles>

Figure S2: Spin labels for protons and phosphorus in $[A B C D X]_{2}$ spin system of compound 2 (left), and numbering scheme for ${ }^{13} \mathrm{C}\left\{{ }^{1} \mathrm{H}\right\}$ NMR of 2 (right).

${ }^{1} \mathrm{H}$ NMR $\left(500 \mathrm{MHz}, \mathrm{C}_{6} \mathrm{D}_{6}, \delta\right): \mathrm{ABCD}$ part of $[\mathrm{ABCDX}]_{2}$ spectrum, $7.48\left(\mathrm{~A} / \mathrm{A}^{\prime}, 2 \mathrm{H}\right), 7.42\left(\mathrm{C} / \mathrm{C}^{\prime}, 2 \mathrm{H}\right), 7.09\left(\mathrm{~B} / \mathrm{B}^{\prime}\right.$, $2 H), 4.08\left(D / D^{\prime}, 2 H, 2 \times P H\right)$.

${ }^{13} \mathrm{C}\left\{{ }^{1} \mathrm{H}\right\}$ NMR $\left(126 \mathrm{MHz}, \mathrm{C}_{6} \mathrm{D}_{6}, \delta\right): 144.5\left(\mathrm{t},{ }^{2} J_{\mathrm{CP}}=2.2 \mathrm{~Hz}, \mathrm{qC}-10\right), 140.2$ (t, A part of an AXX' spin system, $N=$ $J_{A X}+J_{A X^{\prime}}=25.6 \mathrm{~Hz}, \mathrm{qC}-1, \mathrm{qC}-9$ ), $134.0(\mathrm{~s}, \mathrm{qC}-5), 130.7$ (t, A part of an AXX' spin system, $N=J_{A X}+J_{A X^{\prime}}=24.4$ $\mathrm{Hz}, \mathrm{C}-2, \mathrm{C}-8$ ), 127.5 (s, C-4, C-6), 127.0 (t, A part of an AXX' spin system, $N=J_{A X}+J_{A X^{\prime}}=7.4 \mathrm{~Hz}, \mathrm{C}-3, \mathrm{C}-7$ ).

${ }^{31} \mathrm{P}$ NMR $\left(202 \mathrm{MHz}, \mathrm{C}_{6} \mathrm{D}_{6}, \delta\right):-104.7$ (X part of $[\mathrm{ABCDX}]_{2}$ spectrum).

${ }^{31} \mathrm{P}\left\{{ }^{1} \mathrm{H}\right\}$ NMR $\left(202 \mathrm{MHz}, \mathrm{C}_{6} \mathrm{D}_{6}, \delta\right):-104.7(\mathrm{~s})$. 


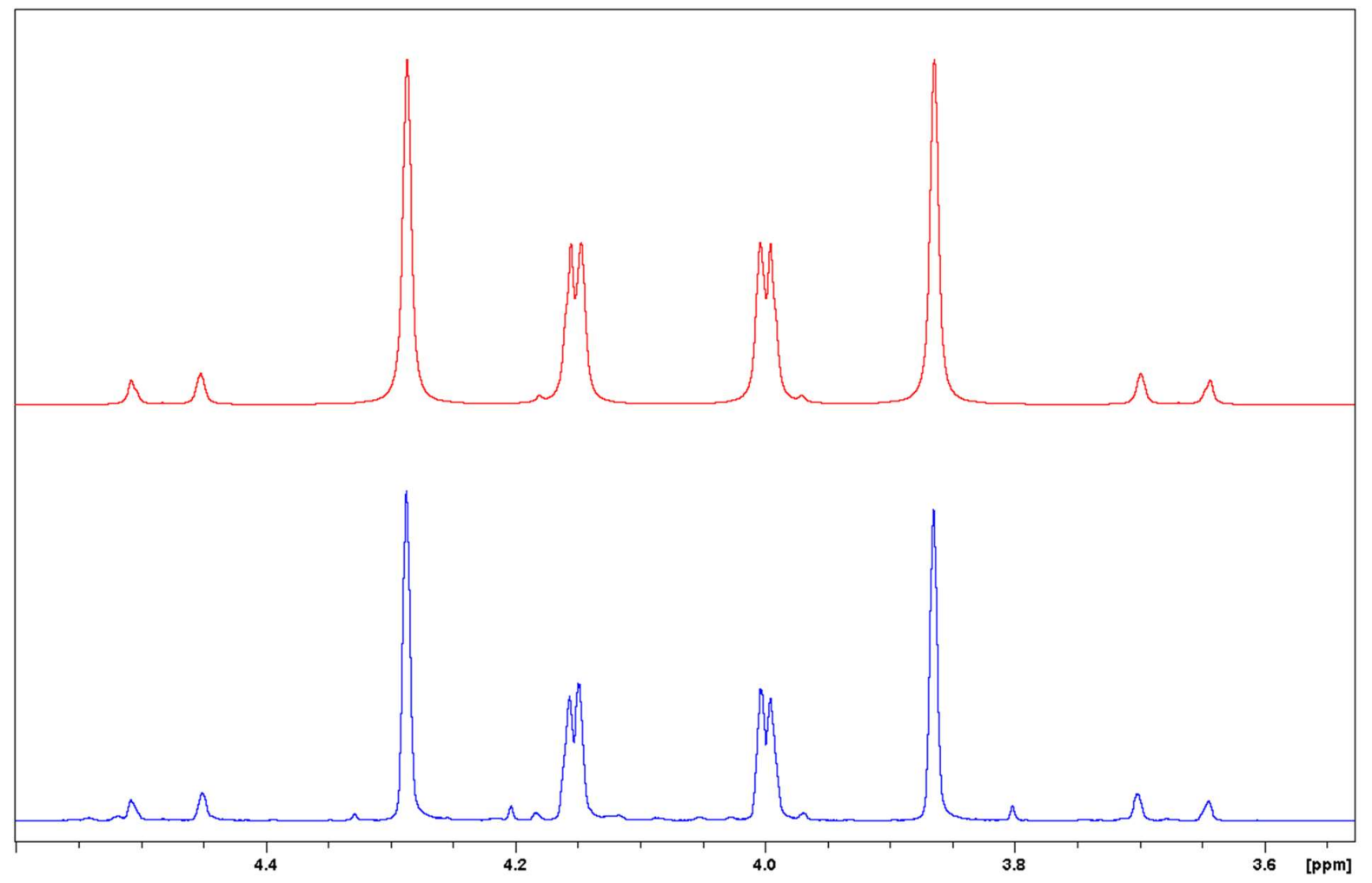

Figure S3: Simulated (top) and experimental (bottom) ${ }^{1} \mathrm{H}$ NMR spectra of 2 showing phosphine region. 


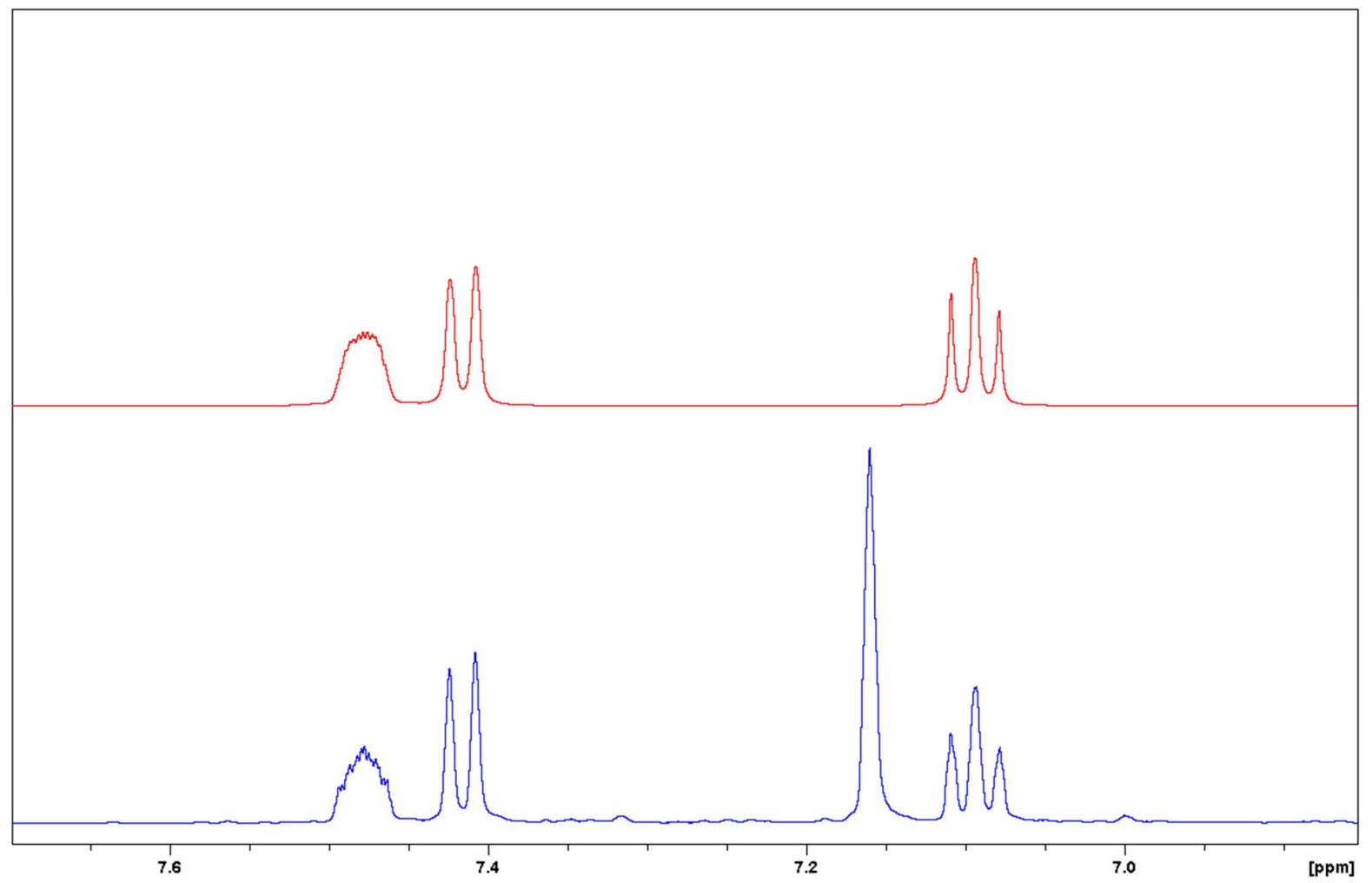

Figure S4: Simulated (top) and experimental (bottom) ${ }^{1} \mathrm{H}$ NMR spectra of 2 showing aromatic region. Peak at $\delta=7.16$ in experimental spectrum is the solvent residual peak for $\mathrm{C}_{6} \mathrm{D}_{6}$. 


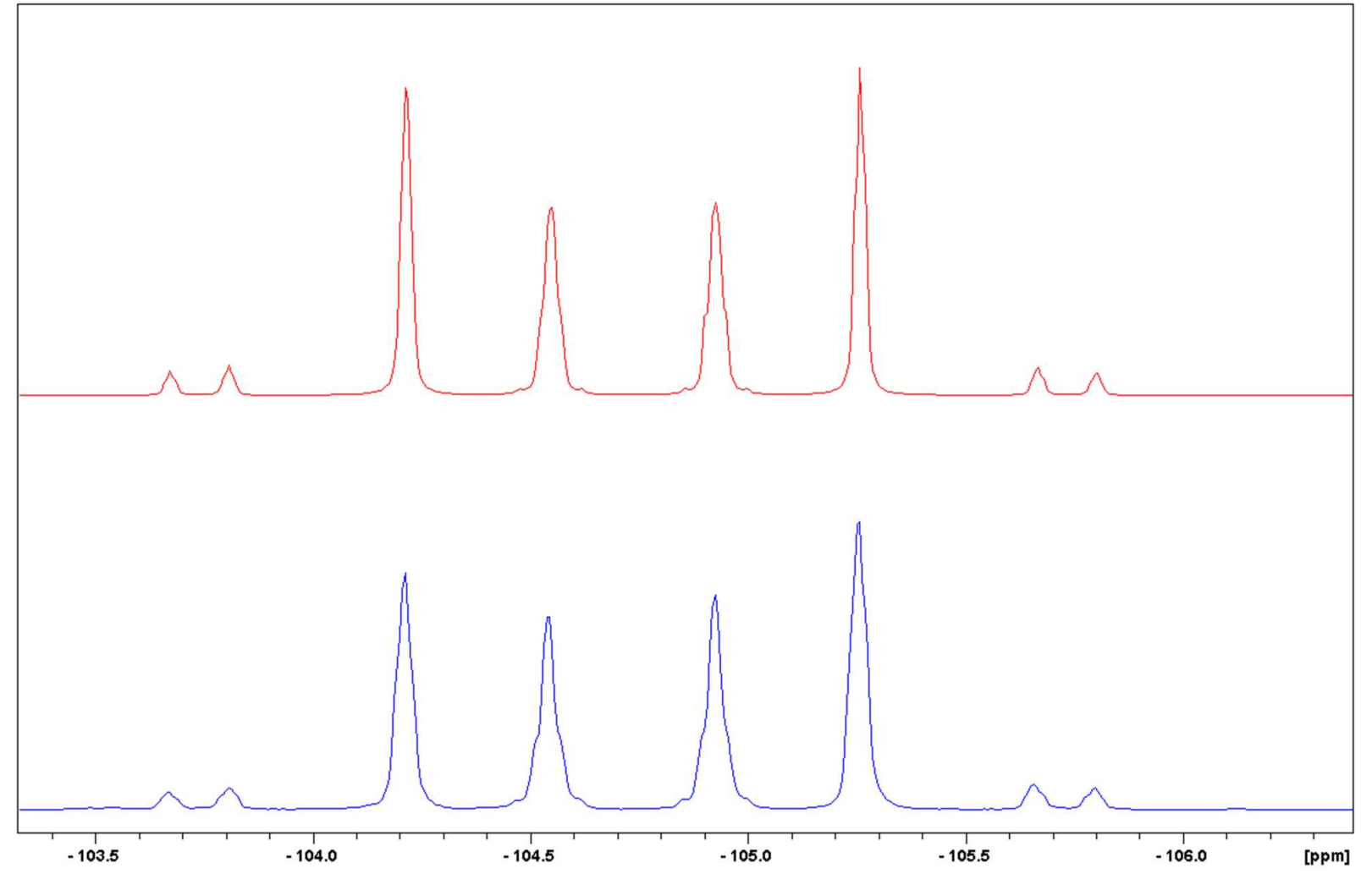

Figure S5: Simulated (top) and experimental (bottom) ${ }^{31} \mathrm{P}$ NMR spectra of 2. 


\section{S6 - Mechanistic Studies \\ S6.1 - Gas Trapping Experiment}

A solution of $5 \mathrm{a}$ in xylenes was heated at $120^{\circ} \mathrm{C}$, under a gentle flow of $\mathrm{N}_{2}$, with an outlet bubbling through a cooled $\left(-78{ }^{\circ} \mathrm{C}\right)$ solution of $\mathrm{CD}_{2} \mathrm{Cl}_{2}$. After heating for $1.5 \mathrm{hrs}$, the ${ }^{1} \mathrm{H}$ NMR spectrum of the $\mathrm{CD}_{2} \mathrm{Cl}_{2}$ solution revealed dissolved propane, which was identified by comparison with literature data. ${ }^{11}$

${ }^{1} \mathrm{H} \mathrm{NMR}\left(500 \mathrm{MHz}, \mathrm{CD}_{2} \mathrm{Cl}_{2}, \delta\right): 1.33\left(\mathrm{sept},{ }^{3} J_{\mathrm{HH}}=7.3 \mathrm{~Hz}, 2 \mathrm{H}\right), 0.90\left(\mathrm{t},{ }^{3} J_{\mathrm{HH}}=7.3 \mathrm{~Hz}, 6 \mathrm{H}\right)$.

\section{S6.2 - Preparation of stock solutions}

Compounds $\mathbf{5 b}$ and $\mathbf{5} \mathbf{d}$ were synthesised and purified as detailed above. All stock solutions were prepared in volumetric flasks under an atmosphere of dry argon, using dry and degassed solvents. The stock solutions were stored under argon in sealed bottles while not in use. The following solutions were prepared:
A. $52.8 \mathrm{mM}$ solution of $\mathbf{5 b}$ in xylenes (isomer mixture).
B. $52.8 \mathrm{mM}$ solution of $\mathbf{5 b}$ in benzonitrile
C. $53.4 \mathrm{mM}$ solution of $\mathbf{5 b}$ in xylenes (isomer mixture). Also contains 1,4-cyclohexadiene (approximately $500 \mathrm{mM}$ ).
D. $53.6 \mathrm{mM}$ solution of $\mathbf{5 b}$ in benzene. Also contains AIBN ( $5.5 \mathrm{mM})$.
E. $53.6 \mathrm{mM}$ solution of $\mathbf{5 b}$ in benzene. Also contains AIBN $(0.25 \mathrm{mM})$.
F. $\quad 45.5 \mathrm{mM}$ solution of $\mathbf{5 d}$ in xylenes (isomer mixture). Also contains $\mathbf{5 b}(6.1 \mathrm{mM})$ and $\mathrm{Ph}_{3} \mathrm{P}(19.1$ $\mathrm{mM}$ ) as an internal standard
G. $51.2 \mathrm{mM}$ solution of $\mathbf{5 b}$ in xylenes (isomer mixture). Also contains $\mathrm{Ph}_{3} \mathrm{P}(16.8 \mathrm{mM})$ as an internal standard

\section{S6.3 - Methodology of Kinetic Experiments}

\section{S6.3.1 - General considerations}

The progress of the reaction was monitored by taking aliquots of the reaction mixture at pre-determined time points, and analysing them by quantitative ${ }^{31} \mathrm{P}\left\{{ }^{1} \mathrm{H}\right\}$ NMR spectroscopy. All kinetic runs were carried out under an atmosphere of dry argon or nitrogen.

All NMR samples were run on a Bruker Avance III 500 MHz NMR spectrometer equipped with a CryoProbe Prodigy BBO; recording a ${ }^{31} \mathrm{P}\left\{{ }^{1} \mathrm{H}\right\}$ NMR spectrum with inverse-gated decoupling (16 scans, pulse delay 45 seconds). The length of pulse delay was chosen based on T1 measurements of compounds $\mathbf{5 b}$ and $\mathbf{6 b}$, to ensure full relaxation between pulses. A sealed glass capillary of $d_{6}$-benzene or $d_{6}$-DMSO was added to each sample to provide a lock. 


\section{S6.3.2 - Typical experimental procedure}

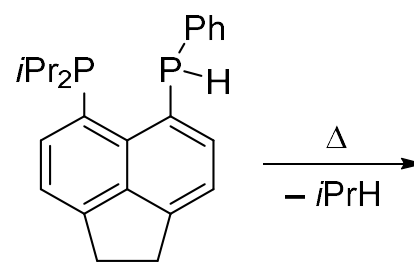

5b<smiles>CC(C)P1c2ccc3c4c(ccc(c24)P1[PH+]c1ccccc1)CC3</smiles>

6b

Scheme S15: General scheme for reaction under study

A $50 \mathrm{~mL}$, 3-necked round bottom flask was charged with a magnetic stirrer and $10 \mathrm{~mL}$ of stock solution A, $B, C, D$, or $E$ (see above). The two side necks were equipped with a mercury thermometer in a Quickfit ${ }^{\circledR}$ adaptor; and a rubber septum. The central neck was equipped with a Liebig condenser and mini-bubbler. A steady, gentle flow of nitrogen was maintained throughout the reaction.

A magnetic stirrer/hotplate, equipped with temperature probe, was used to heat a silicone oil bath to a predetermined temperature, $T_{\text {bath. }}$. The temperature probe was used to ensure the temperature remained steady (Figure S6).

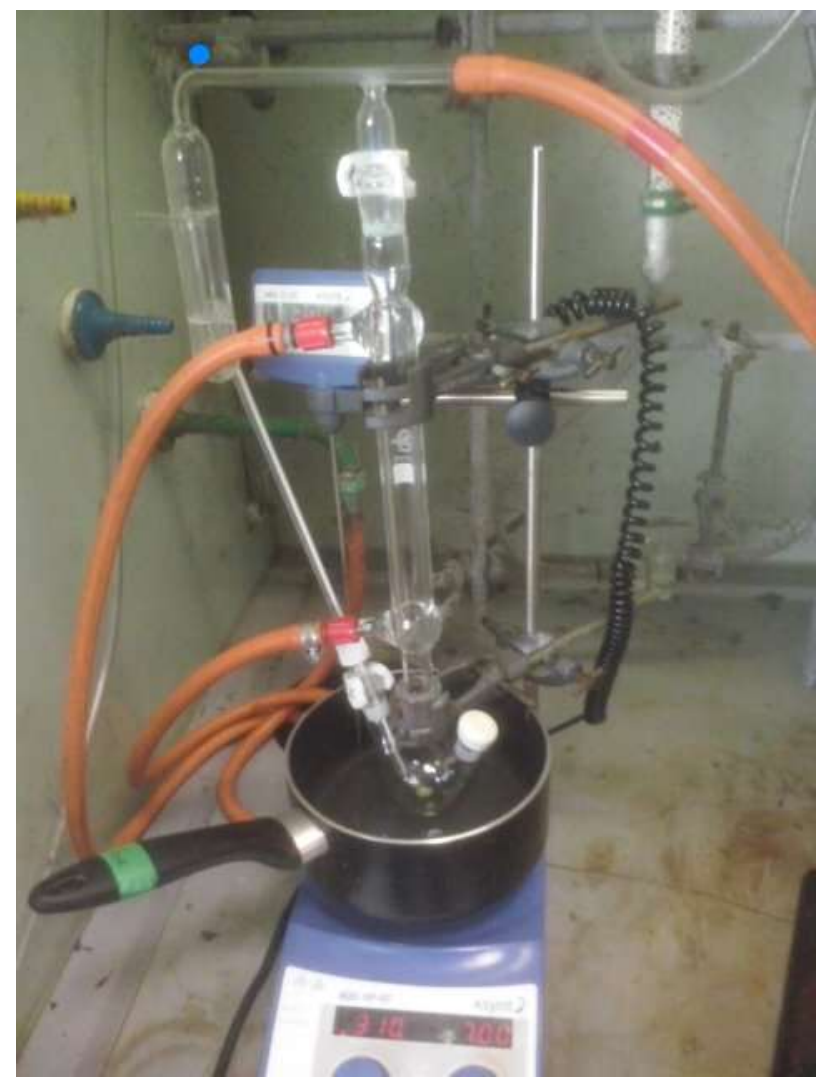

Figure S6: Photograph of equipment used for kinetic experiments 
Once the desired $T_{\text {bath }}$ was reached, the reaction was lowered into the oil bath, and a timer was started. At predetermined time points, the temperature on the thermometer in the reaction flask was read $\left(T_{\mathrm{rxn}}\right)$, and an aliquot $(\approx 0.45 \mathrm{~mL}$ ) of reaction solution was removed by syringe via the rubber septum.

The aliquot was transferred to an NMR tube containing a sealed glass capillary of $d_{6}$-benzene and the tube was sealed with a pressure cap. 


\section{S6.4 - Kinetic Data}

In the main paper (section 2.3), two Eyring plots are produced (Figure 2, $\mathrm{C}$ and D). We state that plotting data points over the temperature range $114-131{ }^{\circ} \mathrm{C}$ gives a better linear fit, and use the activation parameters obtained from graph $D$.

This is because while reactions in the range $114-131{ }^{\circ} \mathrm{C}$ gave data that clearly fit second order kinetics, the measurements at lower temperatures were less clear. When data obtained in the range $94-103^{\circ} \mathrm{C}$ were plotted, a very slight curvature was observed in the second order rate plots (see below). This could be due to systematic error, or could indicate a change in mechanism.

Run 1: Stock solution A (solvent $=$ xylenes, see section S6.2), $T_{\text {rxn }}=131 \pm 1{ }^{\circ} \mathrm{C}, T_{\text {bath }}=140 \pm 1{ }^{\circ} \mathrm{C}$

\begin{tabular}{ccc}
\hline time $(\mathbf{h})$ & {$[\mathbf{A}](\mathbf{m M})$} & $\mathbf{1 / [ A ]}\left(\mathbf{M}^{-\mathbf{1}}\right)$ \\
\hline 0.25 & 29.3 & 34.2 \\
0.50 & 20.9 & 48.0 \\
0.75 & 16.2 & 61.9 \\
1.00 & 13.4 & 74.8 \\
1.50 & 9.7 & 102.6 \\
2.00 & 7.7 & 130.2 \\
3.00 & 5.3 & 188.6 \\
4.00 & 3.8 & 264.8 \\
\hline
\end{tabular}

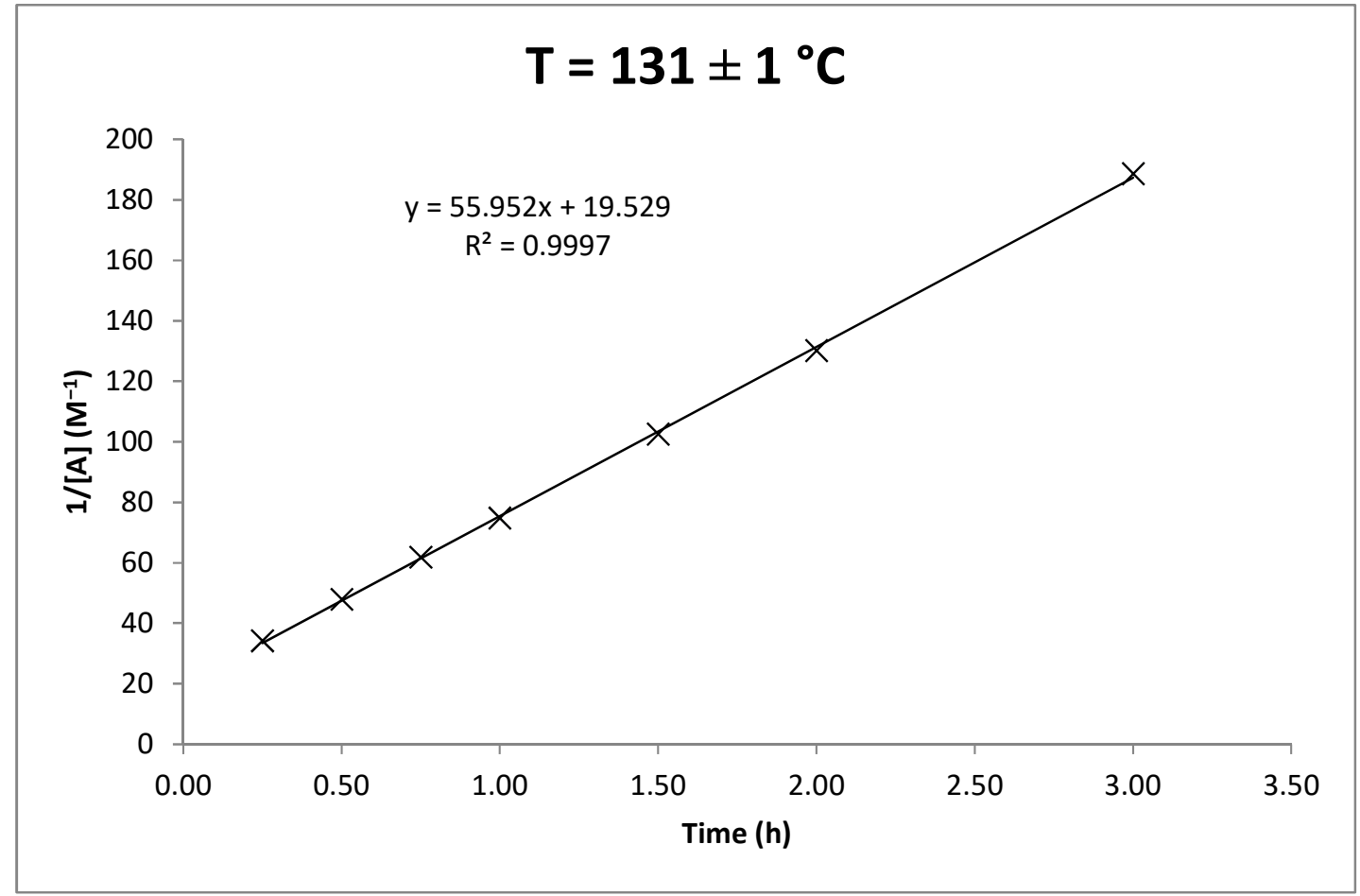


Run 2: Stock solution A (solvent = xylenes, see section S6.2), $T_{\text {rxn }}=131 \pm 1{ }^{\circ} \mathrm{C}, T_{\text {bath }}=140 \pm 1{ }^{\circ} \mathrm{C}$

\begin{tabular}{ccc}
\hline time $(\mathbf{h})$ & {$[\mathbf{A}](\mathbf{m M})$} & $\mathbf{1 / [ A ]}\left(\mathbf{M}^{-1}\right)$ \\
\hline 0.25 & 29.1 & 34.4 \\
0.50 & 20.4 & 48.9 \\
0.75 & 16.0 & 62.7 \\
1.00 & 13.2 & 75.9 \\
1.50 & 9.6 & 104.5 \\
2.00 & 7.5 & 133.2 \\
2.50 & 6.0 & 165.8 \\
3.00 & 5.2 & 193.3 \\
\hline
\end{tabular}

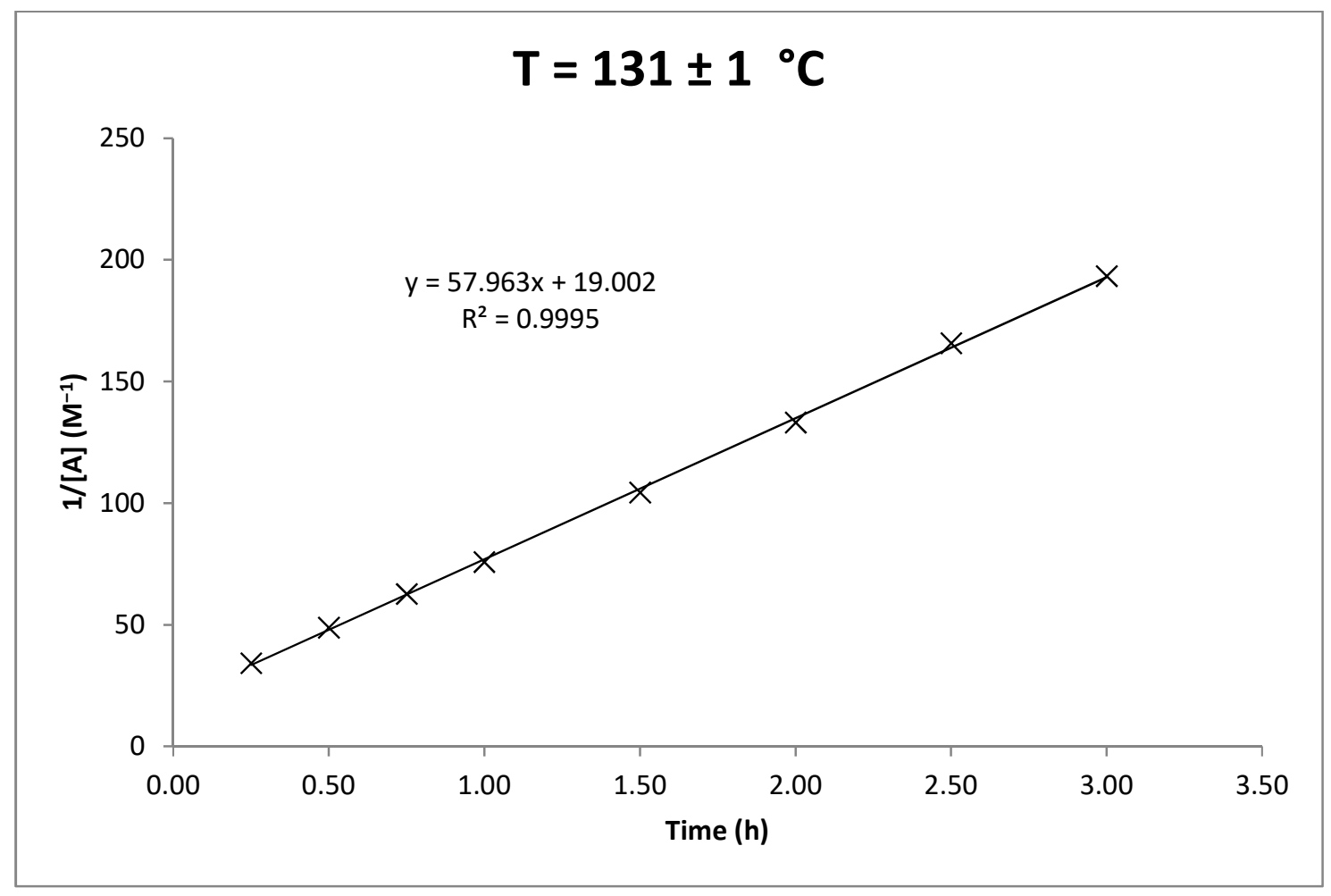


Run 3: Stock solution A (solvent $=$ xylenes, see section S6.2), $T_{\text {rxn }}=131 \pm 1{ }^{\circ} \mathrm{C}, T_{\text {bath }}=140 \pm 1{ }^{\circ} \mathrm{C}$

\begin{tabular}{ccc}
\hline time $(\mathrm{h})$ & {$[\mathrm{A}](\mathbf{m M})$} & $\mathbf{1 / [ A ]}\left(\mathbf{M}^{-1}\right)$ \\
\hline 0.25 & 29.9 & 33.4 \\
0.50 & 20.9 & 47.8 \\
0.75 & 16.0 & 62.3 \\
1.00 & 13.1 & 76.1 \\
1.50 & 9.6 & 104.6 \\
2.00 & 7.5 & 133.6 \\
\hline
\end{tabular}

\section{$\mathrm{T}=131 \pm 1{ }^{\circ} \mathrm{C}$}

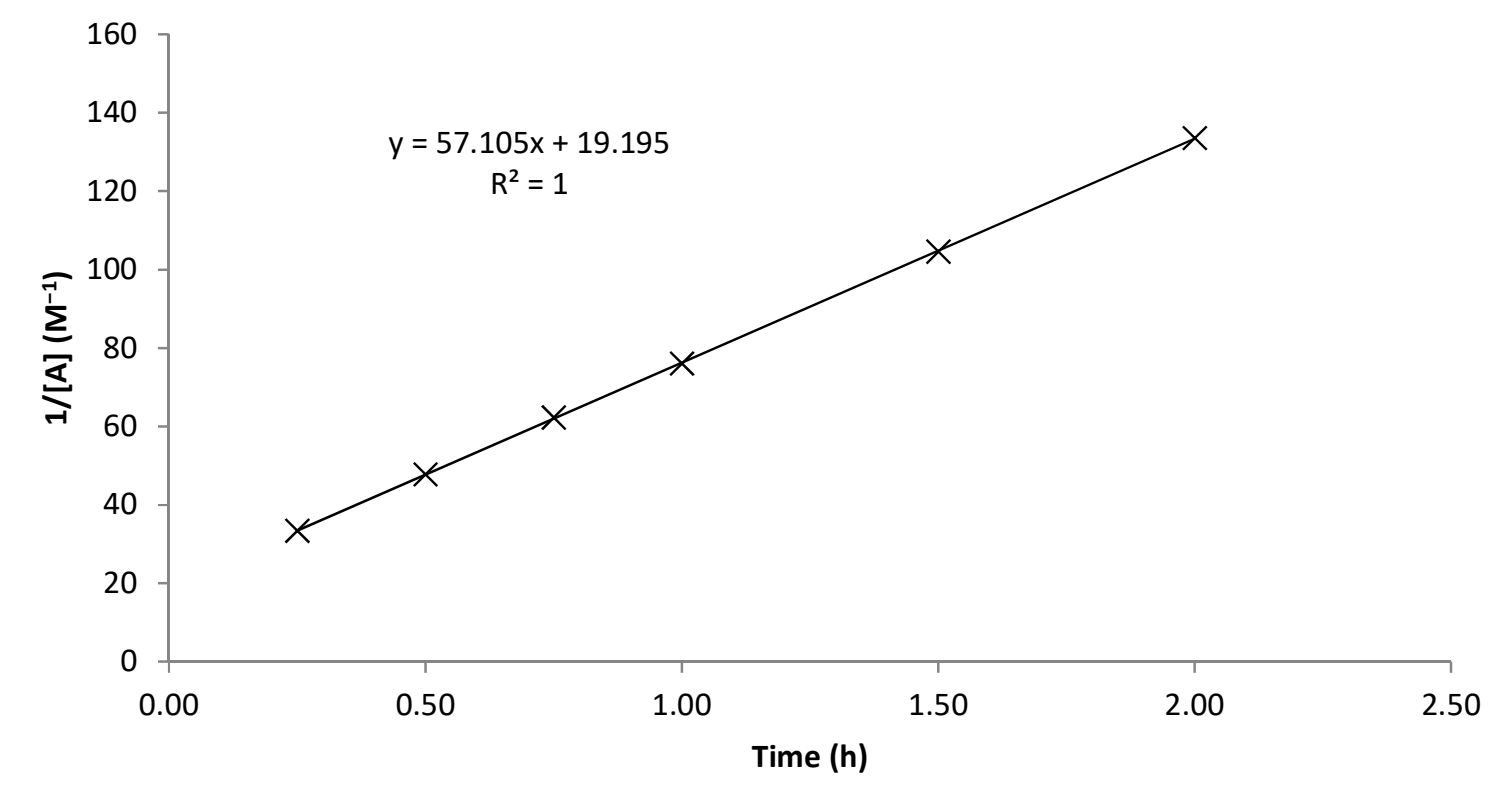


Run 4: Stock solution A (solvent $=$ xylenes, see section S6.2), $T_{\text {rxn }}=121 \pm 1{ }^{\circ} \mathrm{C}, T_{\text {bath }}=130 \pm 1{ }^{\circ} \mathrm{C}$

\begin{tabular}{ccc}
\hline time $(\mathrm{h})$ & {$[\mathrm{A}](\mathrm{mM})$} & $\mathbf{1 / [ A ]}\left(\mathbf{M}^{-1}\right)$ \\
\hline 0.25 & 34.9 & 28.7 \\
0.50 & 26.1 & 38.3 \\
0.75 & 21.0 & 47.5 \\
1.00 & 17.5 & 57.3 \\
1.50 & 13.2 & 75.9 \\
2.00 & 10.4 & 95.9 \\
\hline
\end{tabular}

\section{$\mathrm{T}=121 \pm 1{ }^{\circ} \mathrm{C}$}

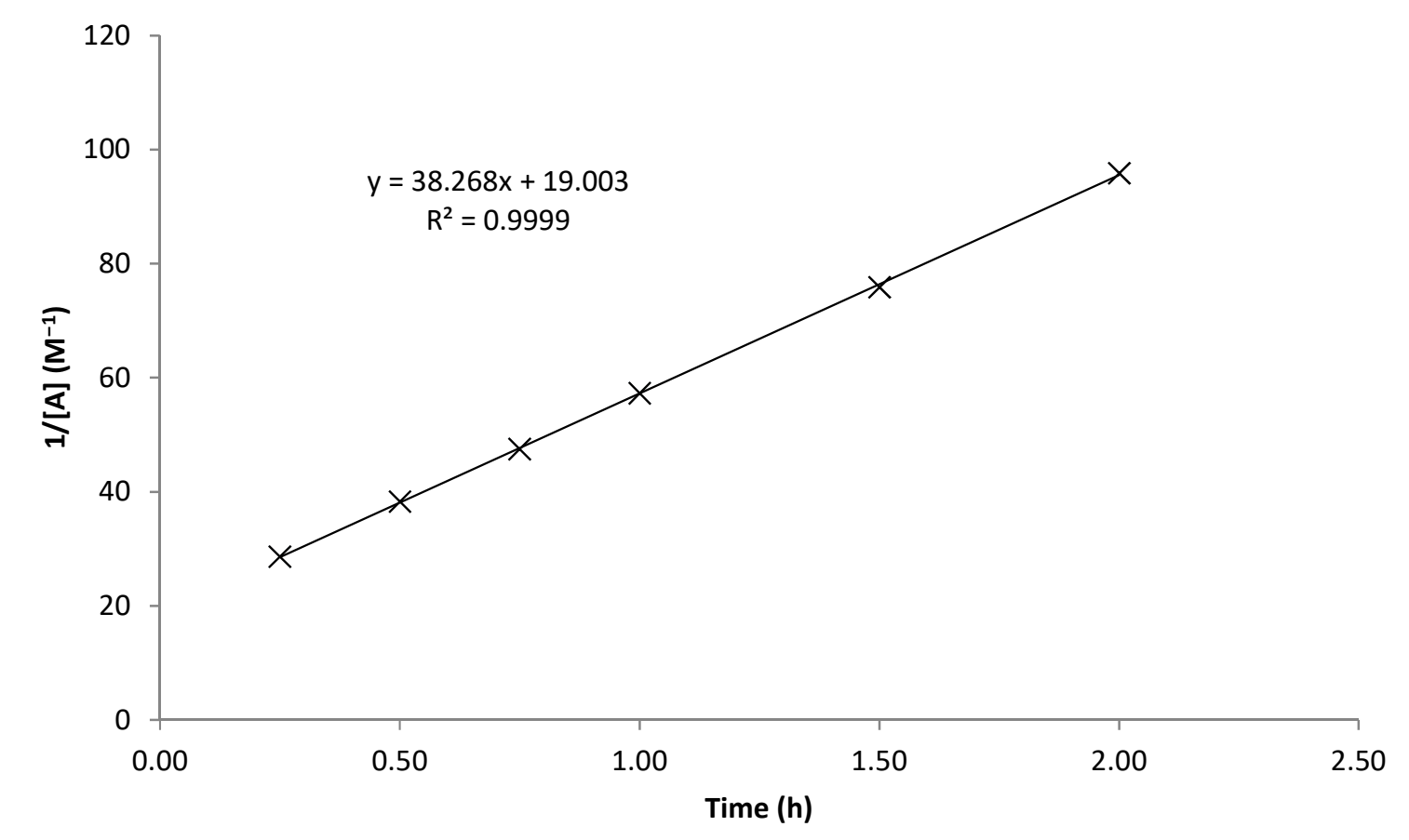


Run 5: Stock solution A (solvent $=$ xylenes, see section S6.2), $T_{\text {rxn }}=114 \pm 1{ }^{\circ} \mathrm{C}, T_{\text {bath }}=120 \pm 1{ }^{\circ} \mathrm{C}$

\begin{tabular}{ccc}
\hline time (h) & {$[\mathbf{A}](\mathbf{m M})$} & $\mathbf{1 / [ A ]}\left(\mathbf{M}^{-1}\right)$ \\
\hline 0.25 & 39.6 & 25.2 \\
0.50 & 32.1 & 31.2 \\
1.00 & 23.3 & 42.9 \\
2.00 & 14.8 & 67.5 \\
3.00 & 10.7 & 93.2 \\
4.00 & 8.4 & 119.6 \\
\hline
\end{tabular}

\section{$\mathrm{T}=114 \pm 1{ }^{\circ} \mathrm{C}$}

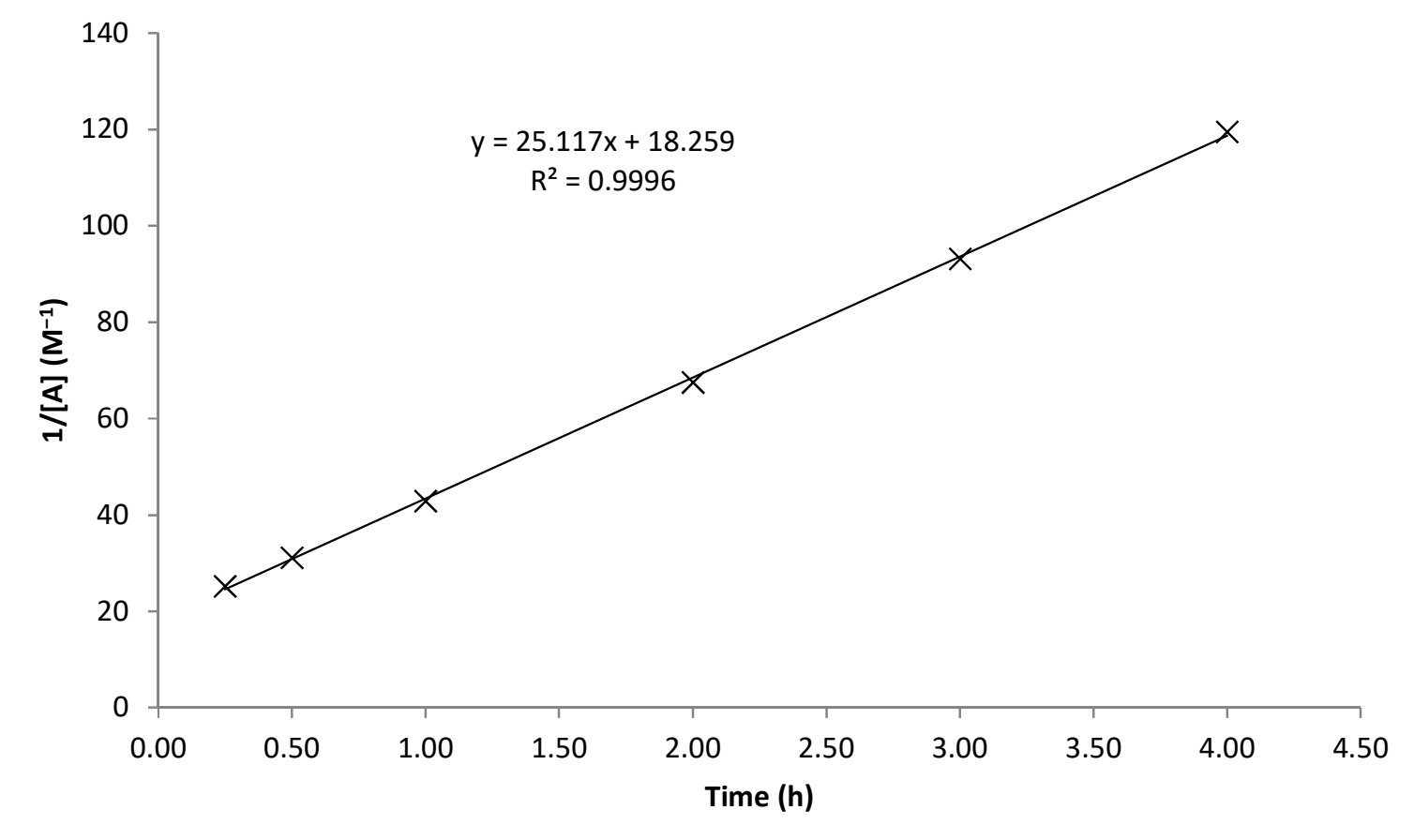


Run 6: Stock solution A (solvent $=$ xylenes, see section S6.2), $T_{\text {rxn }}=103 \pm 1^{\circ} \mathrm{C}, T_{\text {bath }}=110 \pm 1{ }^{\circ} \mathrm{C}$

\begin{tabular}{ccc}
\hline time (h) & {$[\mathrm{A}](\mathbf{m M})$} & $\mathbf{1 / [ A ]}\left(\mathbf{M}^{-1}\right)$ \\
\hline 0.25 & 47.5 & 21.1 \\
0.50 & 43.0 & 23.2 \\
1.00 & 36.2 & 27.7 \\
2.00 & 27.4 & 36.4 \\
3.00 & 21.7 & 46.2 \\
4.00 & 17.6 & 56.7 \\
5.00 & 14.6 & 68.7 \\
6.00 & 12.4 & 80.5 \\
\hline
\end{tabular}

\section{$\mathrm{T}=103 \pm 1{ }^{\circ} \mathrm{C}$}

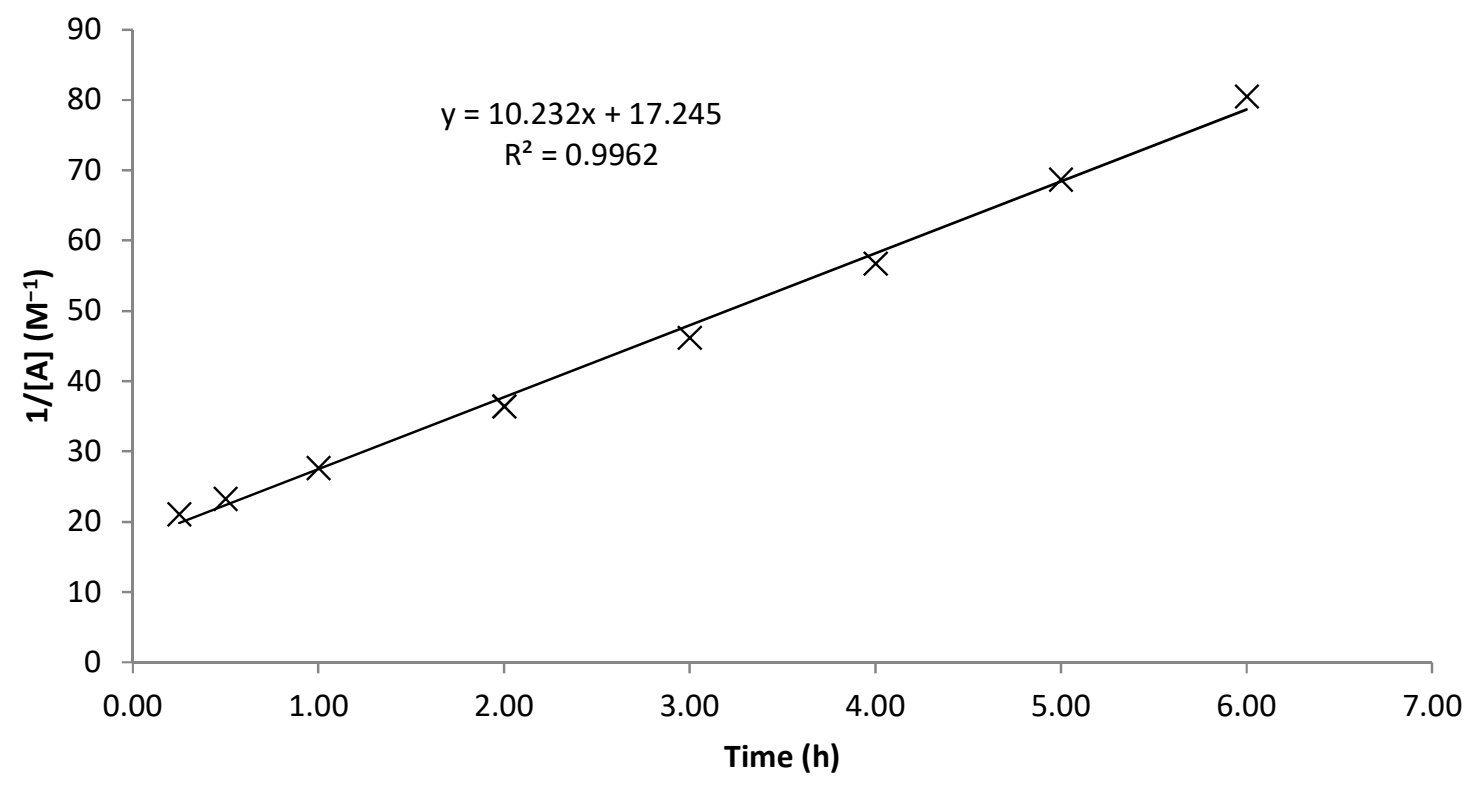


Run 7: Stock solution A (solvent = xylenes, see section S6.2), $T_{\text {rxn }}=94 \pm 1{ }^{\circ} \mathrm{C}, T_{\text {bath }}=100 \pm 1{ }^{\circ} \mathrm{C}$

\begin{tabular}{ccc}
\hline time $(\mathbf{h})$ & {$[\mathbf{A}](\mathbf{m M})$} & $\mathbf{1 / [ A ]}\left(\mathbf{M}^{-1}\right)$ \\
\hline 0.25 & 51.2 & 19.5 \\
0.50 & 48.6 & 20.6 \\
1.00 & 43.7 & 22.9 \\
2.00 & 35.9 & 27.9 \\
3.00 & 30.1 & 33.2 \\
4.00 & 25.9 & 38.6 \\
6.00 & 20.1 & 49.8 \\
8.00 & 16.0 & 62.6 \\
\hline
\end{tabular}

\section{$\mathrm{T}=94 \pm 1{ }^{\circ} \mathrm{C}$}

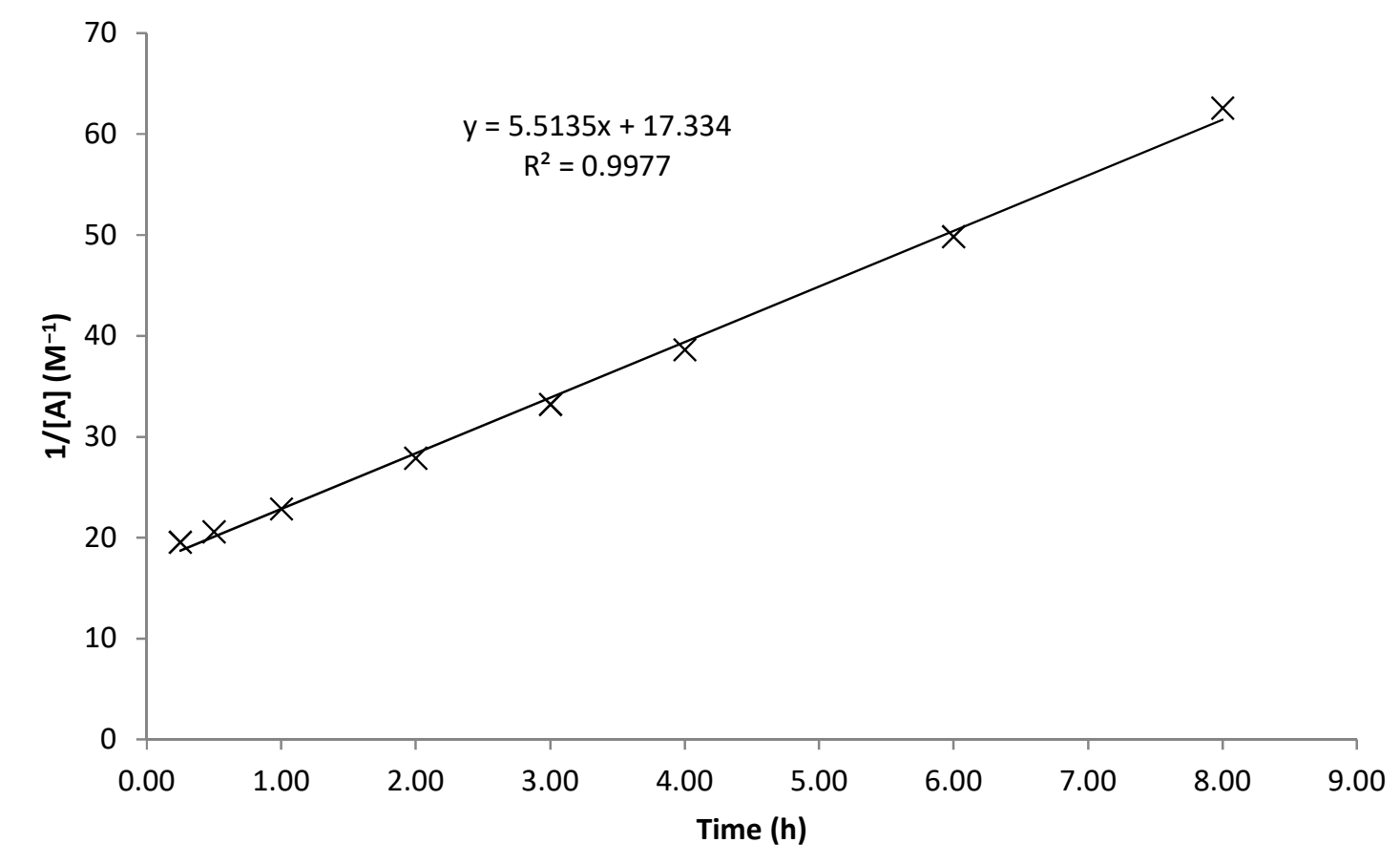


Run 8: Stock solution B (solvent = benzonitrile, see section S6.2), $T_{\text {rxn }}=128 \pm 1{ }^{\circ} \mathrm{C}, T_{\text {bath }}=140 \pm 1^{\circ} \mathrm{C}$

\begin{tabular}{ccc}
\hline time $(\mathbf{h})$ & {$[\mathbf{A}](\mathbf{m M})$} & $\mathbf{1 / [ A ]}\left(\mathbf{M}^{-\mathbf{1}}\right)$ \\
\hline 0.50 & 42.7 & 23.4 \\
0.75 & 39.4 & 25.4 \\
1.00 & 36.8 & 27.2 \\
1.50 & 32.4 & 30.9 \\
2.00 & 28.9 & 34.7 \\
2.50 & 26.1 & 38.3 \\
3.00 & 23.8 & 41.9 \\
\hline
\end{tabular}

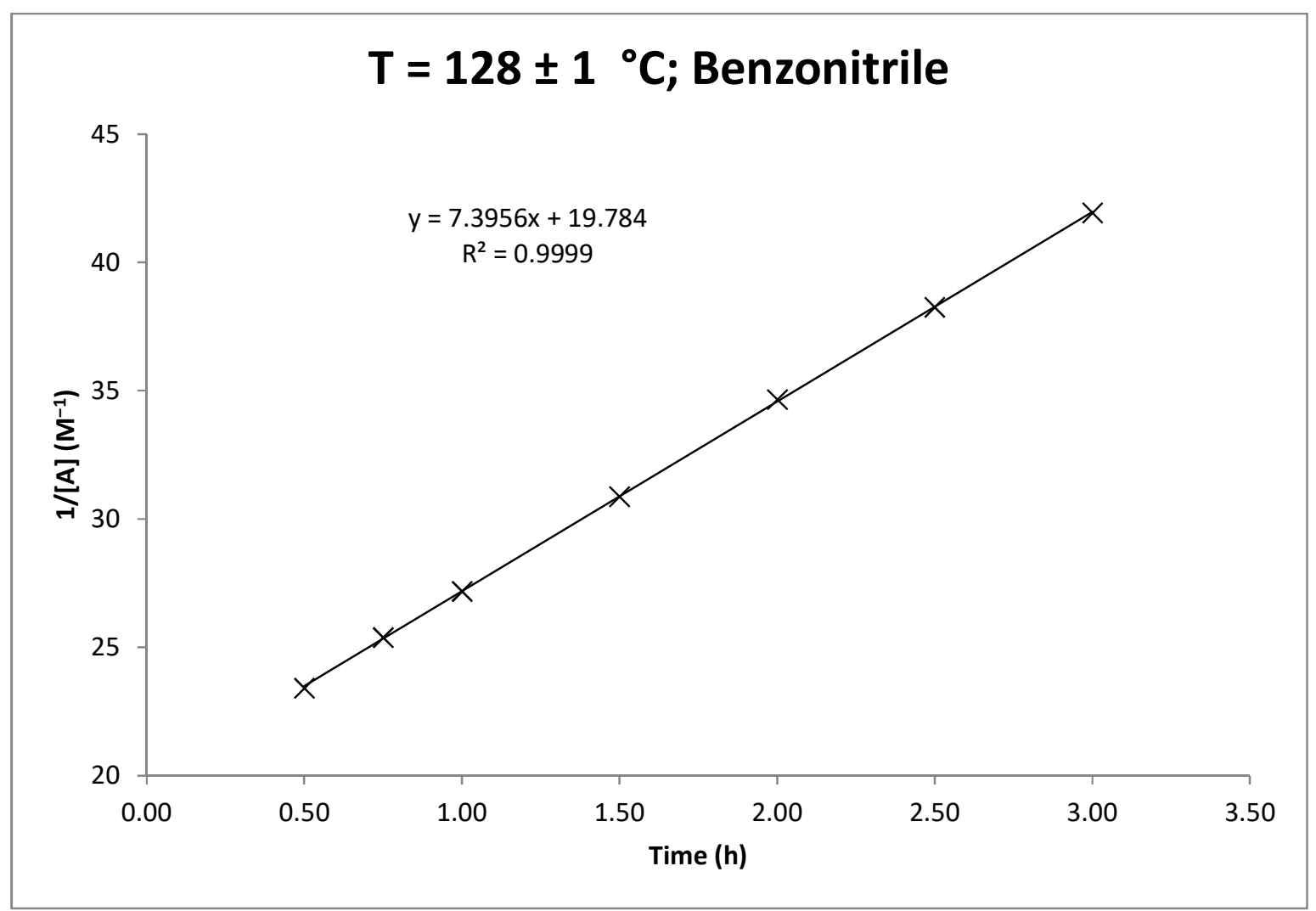


Run 9: Stock solution C (solvent = xylenes, approx. 10 equivalents 1,4-cyclohexadiene, see section S6.2), $T_{\text {rxn }}=129 \pm 1{ }^{\circ} \mathrm{C}, T_{\text {bath }}=140 \pm 1^{\circ} \mathrm{C}$

\begin{tabular}{cc}
\hline time (h) & {$[\mathrm{A}](\mathrm{mM})$} \\
\hline 0.25 & 53.4 \\
0.50 & 53.4 \\
0.75 & 53.4 \\
1.00 & 53.4 \\
1.50 & 52.9 \\
\hline
\end{tabular}

Run 10: Stock solution D (solvent = benzene, approx. $10 \mathrm{~mol} \%$ AIBN, see section S6.2), $T_{\text {rxn }}=72 \pm 1^{\circ} \mathrm{C}, T_{\text {bath }}$ $=80 \pm 1^{\circ} \mathrm{C}$

time (h) $[\mathrm{A}](\mathrm{mM})$

$0.25<0.1$ 
Run 11: Stock solution $\mathrm{E}$ (solvent = xylenes, approx. $0.5 \mathrm{~mol} \% \mathrm{AIBN}$, see section $\mathrm{S} 6.2$ ), $T_{\mathrm{rxn}}=72 \pm 1{ }^{\circ} \mathrm{C}, T_{\text {bath }}$ $=80 \pm 1{ }^{\circ} \mathrm{C}$. Note the graph below is $1^{\text {st }}$ order rate plot.

\begin{tabular}{ccc}
\hline time $(\min )$ & {$[\mathbf{A}](\mathrm{mM})$} & $\operatorname{In}[\mathbf{A}]$ \\
\hline 10 & 37.2 & 3.62 \\
20 & 20.9 & 3.04 \\
30 & 12.0 & 2.48 \\
40 & 7.0 & 1.94 \\
50 & 4.0 & 1.40 \\
60 & 2.3 & 0.85 \\
\hline
\end{tabular}

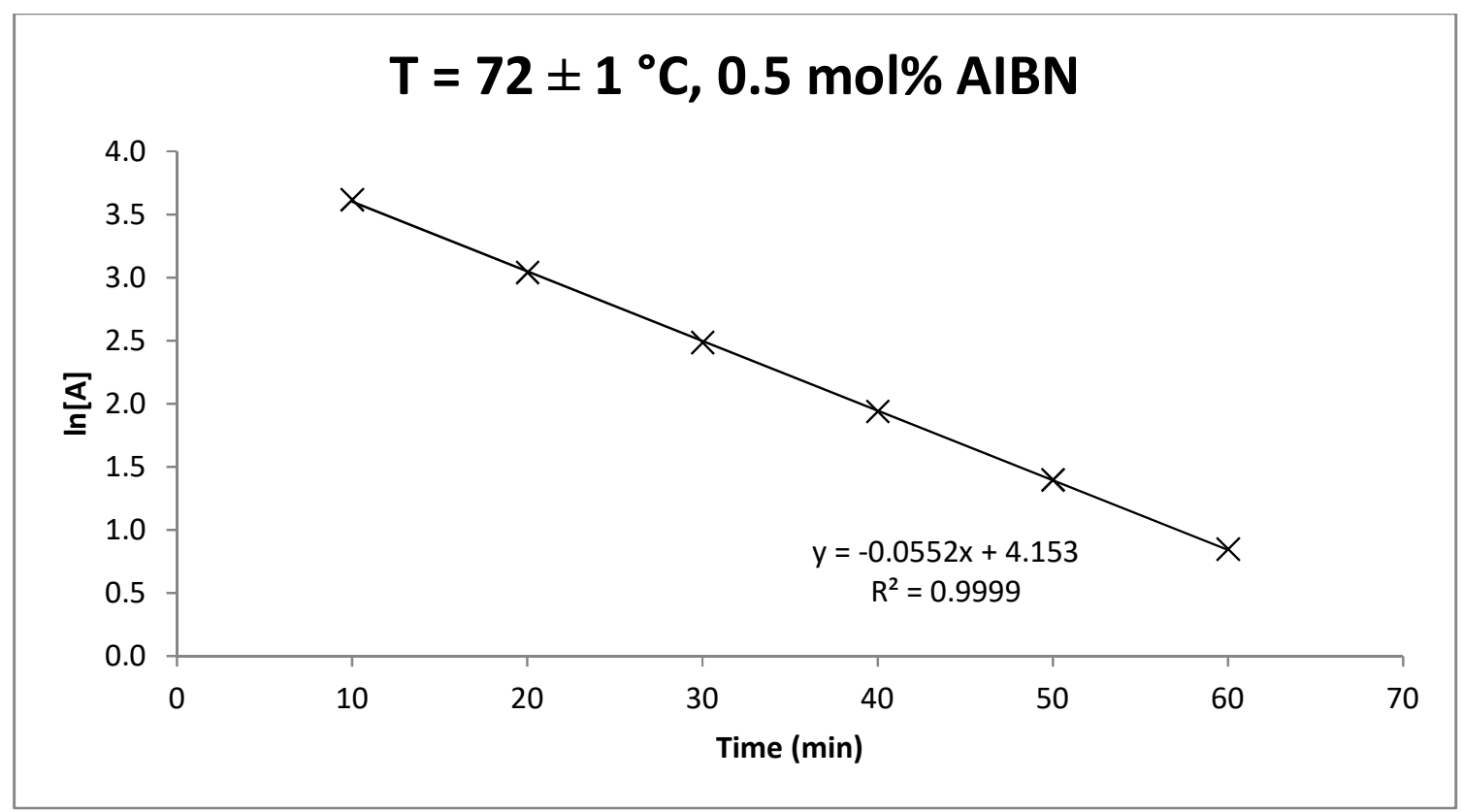




\section{S6.5 - Kinetic Isotope Effect}

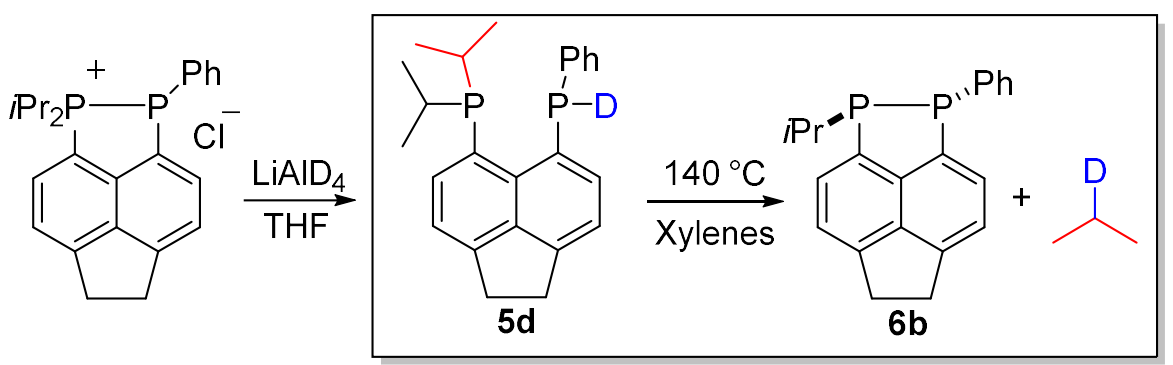

Scheme S16 Synthesis of deuterated compound 5d, and thermal elimination of propane-2-d to afford $\mathbf{6 b}$.

The deuterated analogue of $\mathbf{5 b}$, compound $\mathbf{5} \mathbf{d}$, was synthesised by reduction of the phosphinophosphonium salt $\left[\right.$ Acenap $\left.\left(\mathrm{PiPr}_{2}\right)(\mathrm{PPh})\right][\mathrm{Cl}]^{3}$ with lithium aluminium deuteride (Scheme S16). Compound $\mathbf{5 d}$, somewhat surprisingly, proved difficult to handle due to the labile nature of the P-D bond. Exposure to the slightest trace of moisture resulted in loss of the deuterium label and formation of the unlabelled $\mathbf{5 b}$. Furthermore, it was found that $\mathbf{5} \mathbf{b}$ would not convert back to $\mathbf{5} \mathbf{d}$ on stirring with excess $\mathrm{D}_{2} \mathrm{O}$, even in the presence of a base (LiOD). While this result was unexpected, similar isotopic discrimination against deuterium by phosphorus has been previously observed by Zhang and Mao. ${ }^{12}$

Because of this instability, it was not possible to purify $\mathbf{5} \mathbf{d}$ by recrystallisation from acetonitrile, as this resulted in significant loss of label (even under strictly anhydrous conditions). Also, attempting to monitor the reaction rate of $\mathbf{5} \mathbf{d}$ by the same method employed above was not successful, as taking aliquots always led to some conversion of $\mathbf{5} \mathbf{d}$ to $\mathbf{5} \mathbf{b}$ and subsequent distortion of the measurements.

To circumvent this, stock solutions of $\mathbf{5} \mathbf{d}$ and $\mathbf{5 b}$ ( $F$ and G, see section S6.2) were prepared in flame-sealed NMR tubes under argon, with a glass capillary of $d_{6}$-DMSO inserted for locking. These were then heated in the same preheated oil bath $\left(T_{\text {bath }}=140{ }^{\circ} \mathrm{C}\right.$ ) for the same amount of time (15 minutes), and then analysed by quantitative ${ }^{31} \mathrm{P}\left\{{ }^{1} \mathrm{H}\right\}$ NMR spectroscopy. In solution $\mathrm{F}$, the concentration of $\mathbf{5 d}$ decreased by $5.0 \mathrm{mM}(11 \%$ decrease from starting concentration of $45.5 \mathrm{mM})$. In solution $\mathrm{G}$, the concentration of $\mathbf{5 b}$ decreased by $22 \mathrm{mM}$ ( $56 \%$ decrease from starting concentration of $51.2 \mathrm{mM}$ ). This gives the approximate ratio of initial reaction rates as $\mathrm{k}_{H} / \mathrm{k}_{\mathrm{D}} \approx 5$. While this method does not afford a high degree of accuracy, the difference in rates is substantial, and unlikely to be solely due to error. 


\section{S6.6 - Detection of propane-2-d}

An NMR tube was charged with $0.6 \mathrm{~mL}$ of stock solution $\mathrm{F}$ (see section $\mathrm{S6.2}$ ), and flame sealed to protect the contents from oxygen and moisture. The tube was clamped in a heated oil bath $\left(T_{\text {bath }}=140{ }^{\circ} \mathrm{C}\right)$ and heated for 3 days. After this time, very little $5 \mathbf{d}$ could be detected by ${ }^{31} \mathrm{P}\left\{{ }^{1} \mathrm{H}\right\}$ NMR spectroscopy.

The sample was analysed by ${ }^{2} \mathrm{H}\left\{{ }^{1} \mathrm{H}\right\}$ and ${ }^{2} \mathrm{H}$ NMR, revealing a signal for propane-2- $d$. This was confirmed by comparison with a known sample of propane-2- $d$, which was prepared by quenching a solution of isopropyl magnesium bromide in diethyl ether with $\mathrm{D}_{2} \mathrm{O}$, and trapping the evolved gas in xylenes (Figure S7).

${ }^{2} \mathrm{H}\left\{{ }^{1} \mathrm{H}\right\}$ NMR (77 MHz, xylenes, $\left.\delta\right): 1.28(\mathrm{~s})$.

${ }^{2} \mathrm{H}$ NMR $\delta_{\mathrm{D}}(77 \mathrm{MHz}$, xylenes, $\delta): 1.28(\mathrm{~m})$.
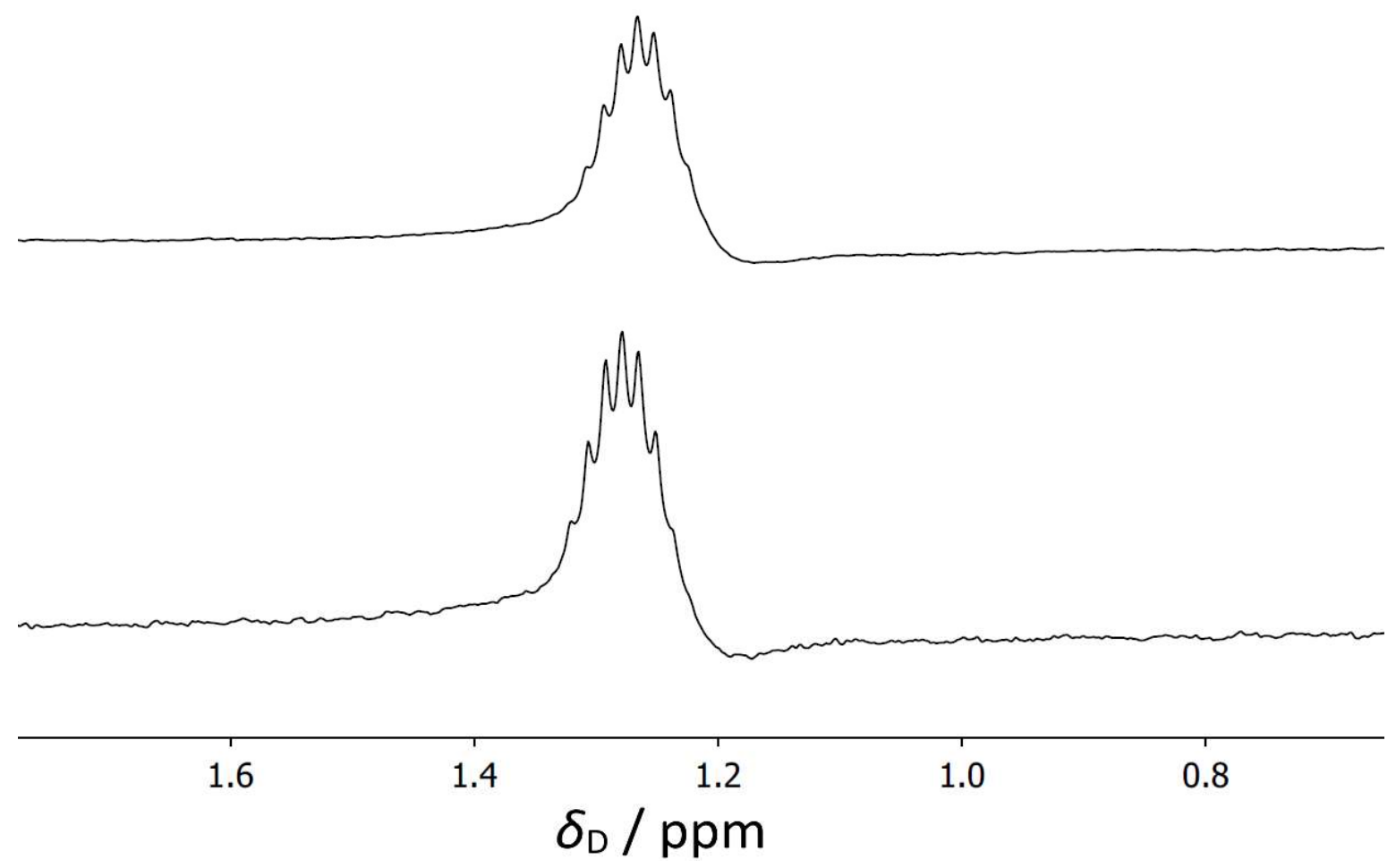

Figure S7: ${ }^{2} \mathrm{H}$ NMR spectra of known sample of propane-2- $d$ (top) and stock solution $\mathrm{F}$ after heating at $140{ }^{\circ} \mathrm{C}$ in a sealed tube for 3 days (bottom). Note that, since ${ }^{2} \mathrm{H}$ NMR spectra are run unlocked and hence without shimming, some distortion of the signal is observed. 


\section{S7 - Mechanistic Discussion}

\section{S7.1 - Reaction in the presence of AIBN}

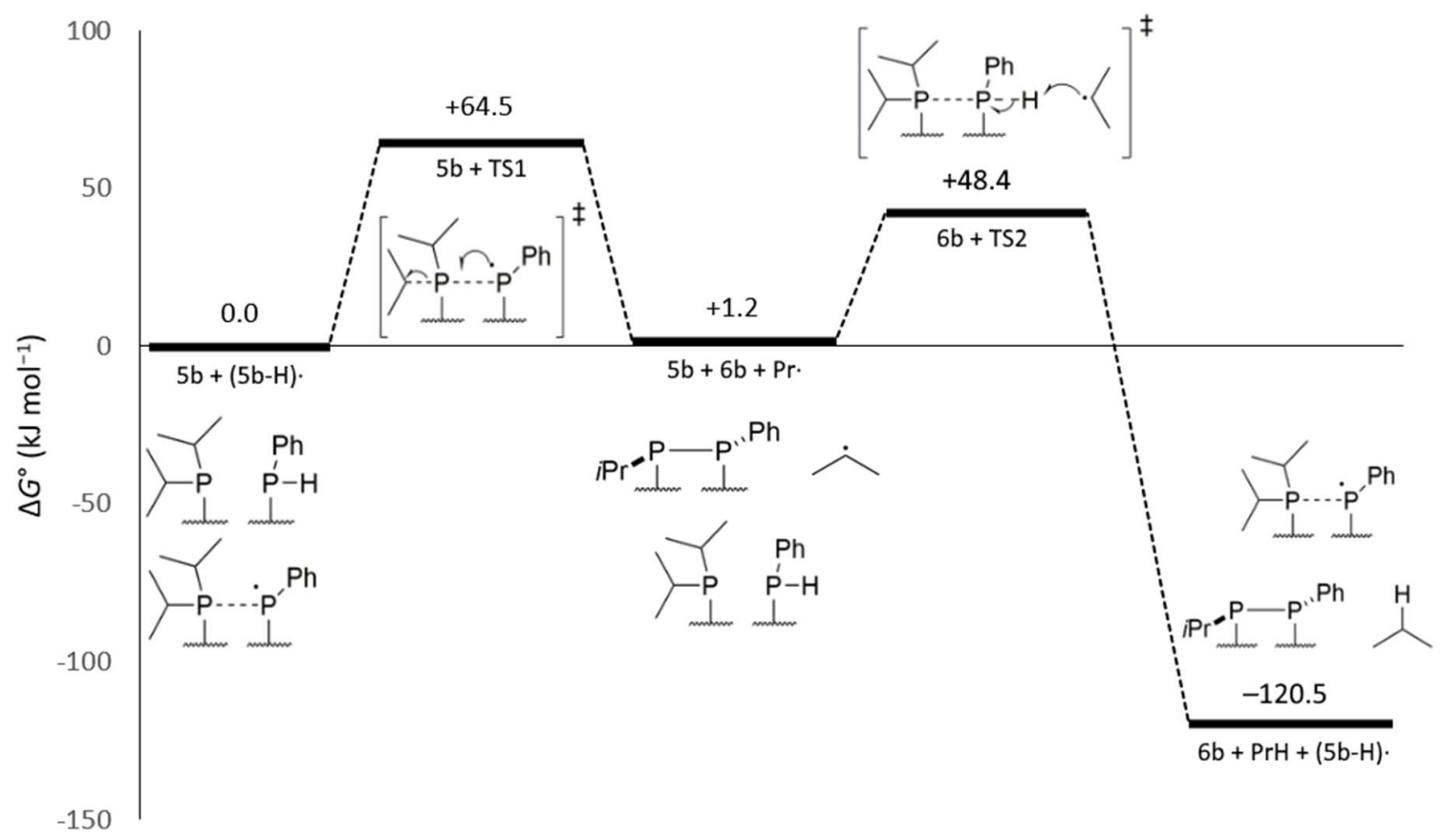

Figure S8: Schematic computed reaction profile for the propagation steps 2 and 3 shown in Scheme 5. TS1 refers to the transition state for radical $(\mathbf{5 b}-\mathrm{H}) \cdot$ undergoing $\mathrm{P}-\mathrm{C}$ bond cleavage to form $\mathrm{Pr}$ - and $\mathbf{6 b}$. TS2 refers to the transition state for $\mathrm{Pr}$ - abstracting hydrogen from $\mathbf{5 b}$. All $\Delta G^{\circ}$ values are computed at $403 \mathrm{~K}$, and given relative to the initial state.

The reaction mechanism depicted in Scheme 5 is analogous, kinetically speaking, to that found in a typical free-radical polymerisation reaction carried out in the presence of a radical initiator. We can therefore write the expected rate equation using a known result from polymer chemistry: ${ }^{13}$

$$
\text { rate }=k[\mathbf{5} \boldsymbol{b}][A I B N]^{\frac{1}{2}}
$$

\section{S7.2 - Initiation without a Radical Initiator}

We believe that unimolecular dissociation of $\mathbf{5 b}$ by homolytic cleavage of the weakest bond $(\mathrm{P}-i \mathrm{Pr})$ is not a plausible initiation step. Not only is the reaction highly energetically unfavourable (Scheme S17, Reaction 1), but it was not possible to construct a rate equation based on this initiation step that predicted second order behaviour (see section S7.4.1). We conclude that a bimolecular initiation step, with 2 molecules of $\mathbf{5 b}$ affording 2 radical species, is better able to account for the observed second order behaviour (see section S7.4.2).

A charge transfer reaction, with 2 molecules of $\mathbf{5 b}$ reacting to give a radical cation and a radical anion, would fulfil this requirement (Scheme S17, Reaction 2). Radical cations (such as the one proposed here) 
are particularly noteworthy, as phosphoniumyl radicals $\left(\mathrm{R}_{3} \mathrm{P}^{+}\right)$have been at the forefront of phosphorus centred radical chemistry. ${ }^{14-16}$ They have been suggested as intermediates in the oxidation of phosphines to their respective oxides, ${ }^{17,18}$ and most recently in FLP chemistry. ${ }^{19}$

However, this initiation step seems unlikely, as the rate of reaction was observed to decrease in a more polar solvent (benzonitrile). If charge separation were occurring, one would expect the opposite effect. Furthermore, the reaction is computed to be very thermodynamically unfavourable $\left(\Delta_{r} G^{\circ}=+405.3 \mathrm{~kJ}\right.$ $\mathrm{mol}^{-1}$ at $403 \mathrm{~K}$ ).

Another option is the bimolecular elimination of a small molecule, either propane or hydrogen (Scheme S17, Reactions 3 and 4). This is more consistent with experimental observations, as no charged species are formed in either of these reactions. Elimination of propane would give rise to two different radicals $((\mathbf{5} \mathbf{b}-\mathrm{H}) \cdot$ and $(\mathbf{5} \mathbf{b}-\mathrm{Pr}) \cdot)$, while elimination of hydrogen would afford two molecules of the same radical, (5bH). Both steps are more thermodynamically favourable than simple unimolecular decomposition of $\mathbf{5 b}$, due mainly to the formation of a strong $(\mathrm{C}-\mathrm{H}$ or $\mathrm{H}-\mathrm{H})$ bond. In addition, both steps are likely to show a strong primary kinetic isotope effect, which would fit with our experimental observations. It should be noted that hydrogen has never been detected as a by-product to the reaction. However, if it is only formed in the initiation step, it is possible that its concentration was too low to be detected by NMR spectroscopy. 
1)<smiles></smiles>

$5 b$
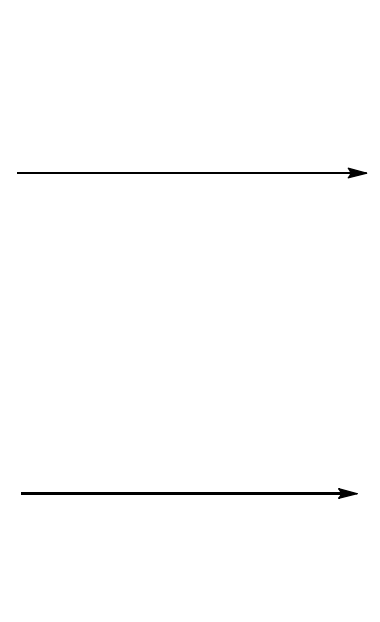<smiles>CC(C)P1c2ccc3c4c(ccc(c24)P1c1ccccc1)CC3</smiles>

(5b-Pr)

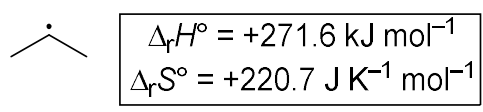

Pr.
2)<smiles>CC(C)P(c1ccc2c3c(ccc(-c4ccccc4)c13)CC2)C(C)C</smiles>

$5 b$<smiles>CC(C)P(c1ccc2c3c(ccc(-c4ccccc4)c13)CC2)C(C)C</smiles>

$5 b$

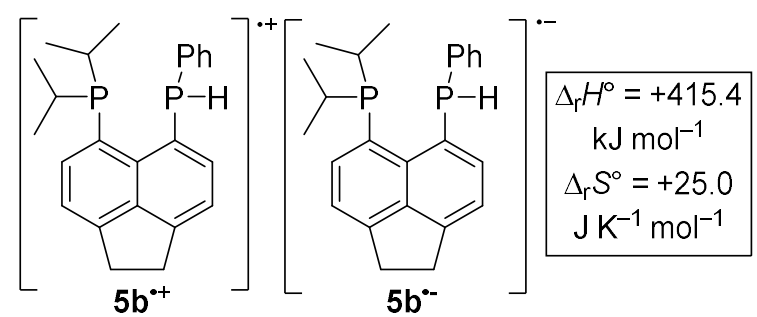

3)<smiles>CC(C)P(c1ccc2c3c(ccc(-c4ccccc4)c13)CC2)C(C)C</smiles>

$5 \mathrm{~b}$<smiles>CC1C=CP1c1ccc2c3c(ccc(-c4ccccc4)c13)CC2</smiles>

$5 b$

$\Delta^{\ddagger} H^{\circ}=+238.2 \mathrm{~kJ} \mathrm{~mol}^{-1}$ $\Delta^{\ddagger} S^{\circ}=-129.5 \mathrm{~J} \mathrm{~K}^{-1} \mathrm{~mol}^{-1}$<smiles>CC(C)P1(C(C)C)=[PH](c2ccccc2)c2ccc3c4c(ccc1c24)CC3</smiles>

(5b-H) •<smiles></smiles>

(5b-Pr) • PrH

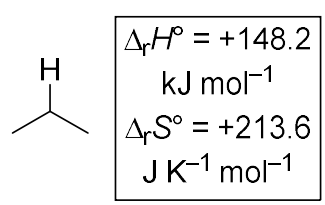

(5b)

$(5 b-H) \cdot$

Scheme S17: Possible initiation steps for the propane elimination reaction, with computed activation parameters and thermodynamic driving forces shown. All these potential initiation steps are unlikely, because they do not fit the experimental data ( 1 and 2), and because they are computed to be too energetically unfavourable (all of these).

The structure of the proposed radical (5b-Pr) - was examined in silico, and was found to be stabilised in a similar way to $(\mathbf{5 b}-\mathrm{H})$. (Figure S9). Formation of the radical again results in a slight decrease in peridistance, as well as an increase in P-P WBI (to 0.14) and significant spin delocalisation (calculated spin densities; $\mathrm{P} 1=0.23, \mathrm{P9}=0.90)$. We thus conclude that $(5 \mathrm{~b}-\mathrm{Pr}) \cdot$ is also a plausible radical intermediate. 

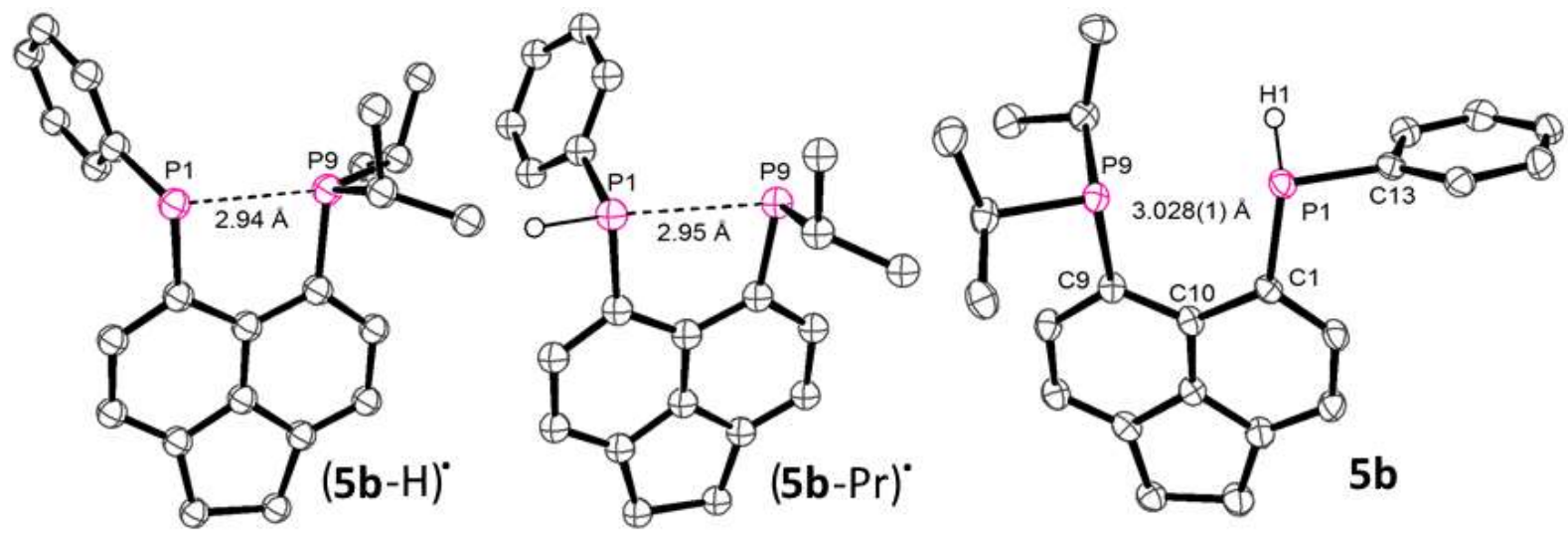

Figure S9: Optimised geometries (B3LYP level) for proposed radical species (5b-H) · (left) and (5b-Pr). (middle), with the solid state structure (from single crystal diffraction) of the starting material (5b) shown on the right for comparison. Carbon bound hydrogen atoms are omitted for clarity. P...P distances are B3LYP optimised, spin density calculated at the M06-2X level.

Although these elimination reactions initially appeared plausible, location of the transition states (Figure S10) revealed them to be prohibitively high in energy. The bimolecular elimination of propane (Reaction 3 , Scheme S17) has a $\Delta^{\ddagger} G^{\circ}$ of ca. $+290 \mathrm{~kJ} \mathrm{~mol}^{-1}$ at $403 \mathrm{~K}$, which is unlikely to be surmountable. Bimolecular elimination of hydrogen (Reaction 4 , Scheme S17) is slightly less disfavoured $\left(\Delta^{\ddagger} G^{\circ}\right.$ ca. $+248 \mathrm{~kJ} \mathrm{~mol}^{-1}$ at $403 \mathrm{~K}$ ) but this is still rather high in energy and likely to be prohibitive. It is worth noting that these computed energy barriers are subject to some uncertainty, due to the complex electronic nature of the transition states (see Sl, section S8.2). It is unlikely, however, that the error associated with these calculations would reduce the computed barrier substantially.
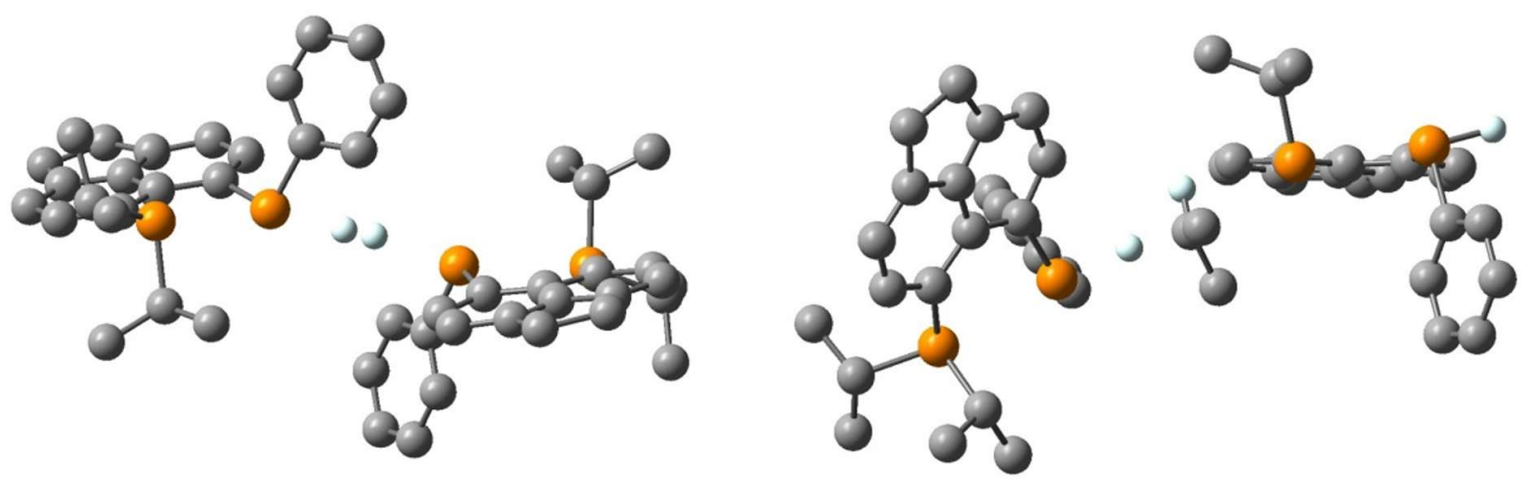

Figure S10: Located initiation transition states for bimolecular elimination of hydrogen (left) and propane (right) from $\mathbf{5 b}$. 


\section{S7.3 - Plausible Termination Steps}

There are several possible termination steps that could be occurring in this system. If the initiation step produces radical (5b-Pr). (see Scheme S17, Figure S9), then the most plausible termination step involves hydrogen abstraction from (5b-Pr). by the propyl radical (Scheme S18, Reaction 1 ). This would afford both observed products, is very thermodynamically favourable $\left(\Delta_{\mathrm{r}} G^{\circ}\right.$ ca. $-302.0 \mathrm{~kJ} \mathrm{~mol}^{-1}$ at $\left.403 \mathrm{~K}\right)$, and is computed to have no barrier. In the absence of $(\mathbf{5 b}-\mathrm{Pr}) \cdot$, several other possible termination steps exist, some of which are shown in Scheme S18. It should be noted that these termination steps involve the formation of by-products that were never observed in the reaction. This includes an experiment where a sample of $\mathbf{5 b}$ in $d_{8}$-toluene was heated in a flame-sealed NMR tube, and then analysed by ${ }^{1} \mathrm{H}$ and ${ }^{31} \mathrm{P}\left\{{ }^{1} \mathrm{H}\right\}$ NMR spectroscopy. Nevertheless, it is possible that these by-products are present in very low concentrations, such that they are difficult to detect. 
1)<smiles>CC(C)CC[PH]1c2ccc3c4c(ccc(c24)P1C(C)C)CC3</smiles>

Pr•

2)<smiles>C=C1C=C(C)[PH](C(C)C)(C(C)C)P1(C)(c1ccccc1)C(C)C</smiles><smiles></smiles>

(5b-H)<smiles>C1CC2CCCC2C1</smiles><smiles>C[12CH3]</smiles>

$\mathrm{Pr} \cdot$

3)

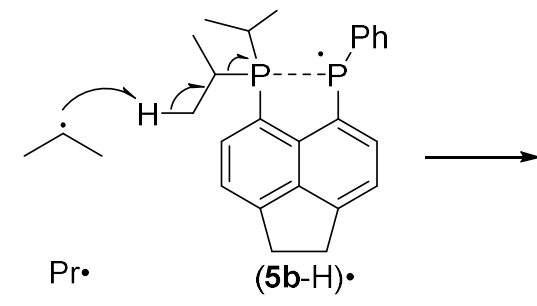

4)<smiles>CC(C)P(c1ccc2c3c(cccc13)CC2)C(C)C</smiles>

$(5 b-H) \cdot$<smiles></smiles>

$6 b$<smiles>CCC(C)C</smiles>
$\Delta_{\mathrm{r}} H^{\circ}=-321.7 \mathrm{~kJ} \mathrm{~mol}^{-1}$ $\Delta_{r} S^{\circ}=-49.0 \mathrm{~J} \mathrm{~K}^{-1} \mathrm{~mol}^{-1}$

PrH

$\Delta_{\mathrm{r}} H^{\circ}=-258.4 \mathrm{~kJ} \mathrm{~mol}^{-1}$ $\Delta_{r} S^{\circ}=-219.6 \mathrm{~J} \mathrm{~K}^{-1} \mathrm{~mol}^{-1}$<smiles>CC(C)P(c1ccccc1)c1ccc2c3c(ccc(P(c4ccccc4)C(C)C)c13)CC2</smiles>

$\Delta_{\mathrm{r}} H^{\circ}=-207.8 \mathrm{~kJ} \mathrm{~mol}^{-1}$ $\Delta_{\mathrm{r}} S^{\circ}=+136.7 \mathrm{~J} \mathrm{~K}^{-1} \mathrm{~mol}^{-1}$

6b $\quad \mathrm{PrH}$

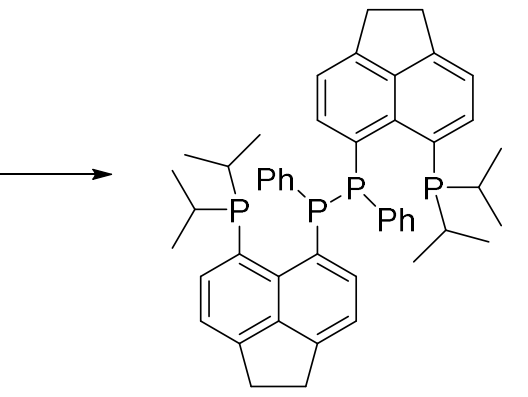

$\Delta_{\mathrm{r}} H^{\circ}=-170.4 \mathrm{~kJ} \mathrm{~mol}^{-1}$ $\Delta_{\mathrm{r}} \mathrm{S}^{\circ}=-223.2 \mathrm{~J} \mathrm{~K}^{-1} \mathrm{~mol}^{-1}$

Scheme S18: Possible termination steps for the radical chain, with thermodynamic parameters shown. 


\section{S7.4 - Derivation of Rate Equations}

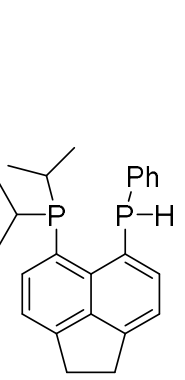

$5 b$
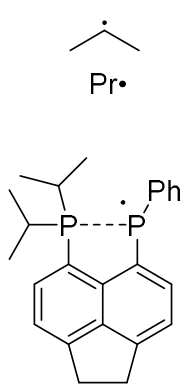

$(5 b-H) \cdot$
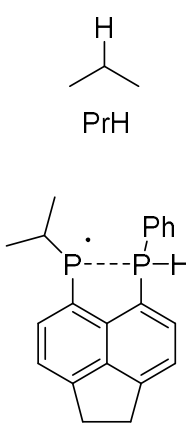

$(5 \mathrm{~b}-\mathrm{Pr}) \cdot$

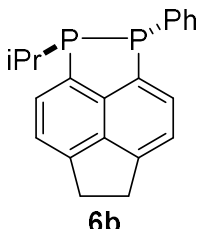

$6 b$

Figure S11: Identification key for all compounds and radical intermediates discussed in this section

\section{S7.4.1 - Unimolecular Initiation}

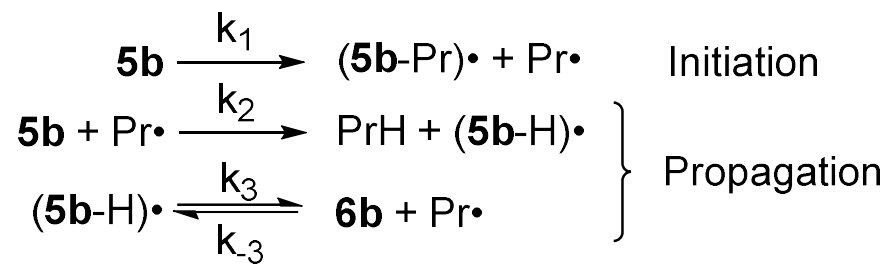

$$
\begin{aligned}
& \mathrm{Pr} \bullet+(5 \mathrm{~b}-\mathrm{Pr}) \cdot \stackrel{\mathrm{k}_{4}}{\longrightarrow} 6 \mathrm{~b}+\mathrm{PrH} \quad \text { Termination }
\end{aligned}
$$

Scheme S19: Abbreviated chain mechanism for reaction initiated by unimolecular dissociation of $\mathbf{5 b}$ via homolytic cleavage of the P-iPr bond.

Scheme $\mathbf{S} 19$ shows a plausible radical chain mechanism, initiated by a unimolecular decomposition of $\mathbf{5 b}$ (Scheme 9, Reaction 1). An identification key for all species involved is given in Figure S11. Applying the steady state approximation to this affords:

$$
\begin{aligned}
k_{1}[\mathbf{5} \boldsymbol{b}] & =k_{4}[(\mathbf{5} \boldsymbol{b}-\operatorname{Pr}) \cdot][\operatorname{Pr} \cdot] \\
k_{3}[(\mathbf{5} \boldsymbol{b}-H) \cdot] & =k_{2}[\mathbf{5} \boldsymbol{b}][\operatorname{Pr} \cdot]+k_{-3}[\mathbf{6} \boldsymbol{b}][\operatorname{Pr} \cdot]
\end{aligned}
$$

These can be substituted into an expression for the rate of $\mathbf{6 b}$ formation:

$$
\begin{gathered}
\frac{d[\mathbf{6} \boldsymbol{b}]}{d t}=k_{3}[(\mathbf{5} \boldsymbol{b}-H) \cdot]-k_{-3}[\mathbf{6} \boldsymbol{b}][\operatorname{Pr} \cdot]+k_{4}[(\mathbf{5} \boldsymbol{b}-\operatorname{Pr}) \cdot][\operatorname{Pr} \cdot] \\
\frac{d[\mathbf{6} \boldsymbol{b}]}{d t}=k_{2}[\mathbf{5} \boldsymbol{b}][\operatorname{Pr} \cdot]+k_{1}[\mathbf{5} \boldsymbol{b}]
\end{gathered}
$$

Clearly, the $k_{1}[\mathbf{5 b}]$ term in equation 4 is first order in $\mathrm{A}$, not second order as required. However, it is possible that this term could be small enough to be neglected, with the rate dominated by the $k_{2}[\mathbf{5} \boldsymbol{b}][\mathrm{Pr} \cdot]$ term. 
For that term to be second order in $\mathbf{5 b}$, the concentration of $\mathrm{Pr}$. must be directly proportional to $\mathbf{5 b}$ (i.e. we can write $[\mathrm{Pr} \cdot]=k[\mathbf{5 b}]$ where $k$ is some unknown constant). If that is the case, then equation 4 implies that the concentration of (5b-Pr). is just a constant, independent of the concentration of $\mathbf{5 b}$. This is clearly unreasonable, as $(\mathbf{5 b}-\mathrm{Pr}) \cdot$ is formed from $\mathbf{5 b}$. Thus, $[\mathrm{Pr} \cdot] \neq k[\mathbf{5 b}]$, and the rate expression cannot be second order in $\mathbf{5 b}$.

A few variations on the chain shown in Scheme S19 have been examined, including additional or alternate termination reactions. Although they resulted in more complex expressions, none of them afforded an expression for the rate of $\mathbf{6} \mathbf{b}$ formation that was plausibly second order in $\mathbf{5 b}$.

\section{S7.4.2 - Bimolecular Initiation}

$$
\begin{aligned}
& 5 \mathbf{b}+5 \mathbf{b} \stackrel{\mathrm{k}_{1}}{\longrightarrow}(5 \mathbf{b}-\mathrm{H}) \cdot+(5 \mathbf{b}-\mathrm{Pr}) \cdot+\mathrm{PrH} \\
& (5 b-H) \cdot \frac{k_{2}}{k_{-2}} 6 b+P r \cdot \\
& \left.\mathbf{5 b}+\mathrm{Pr} \bullet \stackrel{\mathrm{k}_{3}}{\longrightarrow} \mathrm{PrH}+(\mathbf{5 b}-\mathrm{H}) \cdot\right\} \text { Propagation } \\
& \mathrm{Pr} \bullet+(5 \mathrm{~b}-\mathrm{Pr}) \cdot \stackrel{\mathrm{k}_{4}}{\longrightarrow} \mathrm{PrH}+\mathbf{6 b} \quad \text { Termination }
\end{aligned}
$$

Scheme S20: Abbreviated chain mechanism for reaction initiated by bimolecular elimination of propane

Scheme S20 shows a plausible radical chain mechanism, initiated by the bimolecular elimination of propane (Scheme 9, Reaction 3). Although this initiation step was computed to be unreasonably high in energy, it is instructive to look at the rate equation that this mechanism predicts. Applying the steady state approximation affords the following identities:

$$
\begin{gathered}
k_{1}[\mathbf{5} \boldsymbol{b}]^{2}=k_{4}[\operatorname{Pr} \cdot][(\mathbf{5} \boldsymbol{b}-P r) \cdot] \\
k_{2}[(\mathbf{5} \boldsymbol{b}-H) \cdot]=k_{1}[\mathbf{5} \boldsymbol{b}]^{2}+k_{-2}[\mathbf{6} \boldsymbol{b}][\operatorname{Pr} \cdot]+k_{3}[\mathbf{5} \boldsymbol{b}][\operatorname{Pr} \cdot]
\end{gathered}
$$

Substituting into an expression for the rate of $\mathbf{6} \mathbf{b}$ formation gives:

$$
\begin{gathered}
\frac{d[\mathbf{6} \boldsymbol{b}]}{d t}=k_{2}[(\mathbf{5} \boldsymbol{b}-H) \cdot]-k_{-2}[\mathbf{6} \boldsymbol{b}][\operatorname{Pr} \cdot]+k_{4}[(\mathbf{5} \boldsymbol{b}-\operatorname{Pr}) \cdot][\operatorname{Pr} \cdot] \\
\frac{d[\mathbf{6} \boldsymbol{b}]}{d t}=2 k_{1}[\mathbf{5} \boldsymbol{b}]^{2}+k_{3}[\mathbf{5} \boldsymbol{b}][\operatorname{Pr} \cdot]
\end{gathered}
$$

Equation $\mathbf{8}$ is overall second order in $\mathbf{5 b}$ if the concentration of $\mathrm{Pr}$. is directly proportional to $\mathbf{5 b}$. If we substitute $[\mathrm{Pr} \cdot]=k[\mathbf{5 b}]$ into equation 8 , then the concentration of $(\mathbf{5 b}-\mathrm{Pr})$. becomes directly proportional to $\mathbf{5 b}$, which seems reasonable. This means that the mechanism in Scheme S20 affords a rate equation that is plausibly second order in $\mathbf{5 b}$, as required.

Similar analysis has been carried out on some more complex chain mechanisms, involving different initiation steps and alternate termination steps. While some of these get quite complex, it was found that the rate equation could be plausibly second order, so long as the initiation step was bimolecular in $\mathbf{5 b}$. 


\section{S8 - Computational Details}

\section{S8.1 - General Procedures}

Geometries were fully optimized at the B3LYP/6-31G(d) level ${ }^{20,21}$ of density functional theory (DFT), together with a fine integration grid (75 radial shells with 302 angular points per shell); the $\mathrm{H}$ atoms bonded to $P$ were described with $6-31 G(d, p)$ basis. Where available, solid state structures were used as starting points for the optimizations. Open-shell species were treated with the unrestricted Kohn-Sham formalism. For doublet states, spin contamination was negligible in all cases. For transition states involving diradicaloid species, open-shell singlet solutions were sought using the broken-symmetry approach. ${ }^{22}$ In these cases, variable extent of spin contamination was found, up to $\left\langle\hat{S}^{2}\right\rangle \approx 1$ expected for full spatial separation of the two spins. The nature of the minima and transition states was verified by computations of the harmonic frequencies at the same level of theory, which were also used to compute thermodynamic corrections to obtain enthalpies and entropies (at standard pressure and temperature). Single-point energies were refined at the M06-2X level ${ }^{23}$ using 6-311+G(d,p) basis and an ultrafine integration grid (99 radial shells with 590 angular points per shell). Enthalpies were evaluated through adding the thermodynamic corrections from the B3LYP/6-31G(d) level. This combination of levels has performed well in previous computational studies of acenaphthene derivatives with pnictogens in peri-positions. ${ }^{24}$ In these single points, implicit solvation effects were included using the polarizable continuum model in its integral equation formalism (IEF-PCM), ${ }^{25-27}$ employing the parameters of benzene. All computations were performed using the Gaussian09 suite of programs. ${ }^{28}$

\section{S8.2 - Bimolecular Initiation Reactions}

This section applies to the transition states of two possible bimolecular initiation steps which were proposed in section S7.2 (Scheme S17, Reactions 3 and 4).

For the elimination of hydrogen (Figure S10, left) the situation was modelled through a broken-symmetry Kohn-Sham wavefunction, where significant spin density is accumulated on the $P$ atoms in the transition state $\left(+0.67 /-0.75\right.$ on the $\mathrm{P}$ atoms with breaking $\mathrm{P}-\mathrm{H}$ bonds, $\left.\left\langle\hat{\mathrm{S}}^{2}\right\rangle=0.82\right)$. For the elimination of propane Figure S10, right) the optimisation of the transition state was conducted for a closed-shell state, as an attempted optimisation for the broken-symmetry state was not successful. The single-point energy calculation in the continuum was conducted for a broken-symmetry state, again with significant spin density on the P-atoms $\left(\left\langle\hat{S}^{2}\right\rangle=0.74\right)$.

\section{S8.3 - Cartesian Co-ordinates of Optimised Structures and Transition States}

Cartesian coordinates in $\AA$, B3LYP-D3/6-31G*(*) optimised (xyz format)

S8.3.1 - (5b-H)

53

C $-2.26799631082 .1065803073-0.8836582692$

C $-1.04576817051 .4829533706-0.6249553404$

C $-1.05469798130 .1355137606-0.1229101131$ 
C - $-2.3335312226-0.43418405490 .1175542274$

C $-3.55751669390 .2228909469-0.1407969094$

C $-3.52700489551 .4970567867-0.6604175541$

H -2.2560541595 $3.1258551517-1.2620472457$

C $0.0811157349-0.71004436980 .1404247152$

C - $2.5382486037-1.73703677250 .6253680103$

H - $4.43728510952 .0475774738-0.8853775049$

C $-1.4405957806-2.51920571970 .8979143527$

C $-0.1497487205-1.99137479870 .6391769991$

H - $1.5374447576-3.52869287711 .2902240873$

H $0.6963967765-2.64289610130 .8339331784$

P $1.7824496161-0.1081793331-0.2862540598$

P $0.49275904622 .4939267168-0.7303574521$

C 2.44579981210 .42185898851 .4137613733

H 1.60964774381 .0161706341 .8046392827

C $2.7029698545-1.7106383171-0.7335075226$

H $2.6195135012-2.43523435540 .0868010753$

C 3.64648486661 .37187679631 .2687820659

H 3.9120035581 .79362581752 .2467985052

H 4.53435660250 .86035147770 .8817589435

H 3.41209398752 .20253544470 .5955887266

C $2.7239766665-0.71196820792 .408612249$

H $1.8448791616-1.34722502252 .5571207903$

H $3.5556973818-1.34799120192 .0835547807$

H $2.9989793279-0.29436905113 .3861343682$

C $4.1957386577-1.4226360915-0.9666178525$

H $4.7102532937-1.1154735379-0.0511599184$

H $4.696884386-2.3259246233-1.3376952022$

H $4.3371361263-0.6334221681-1.7147754393$

C $2.0761421075-2.3164622965-2.0002343729$

H $2.6122655072-3.2308951352-2.2853919822$

H $1.0224897186-2.5738719544-1.8577008614$

H $2.1403787964-1.6159590382-2.8415395819$

C $-4.7099601179-0.6921193250 .230691183$

H $-5.3552805182-0.8943161253-0.6329531419$

H -5.3516817297-0.23957901810.9964715916

C $-4.0271605231-2.00049750160 .7538621904$

H - $4.3319481216-2.87658813140 .1687305142$

H - $-4.3063316044-2.21104949821 .7935655118$

C $0.65813699632 .8393399003-2.511021415$

C $1.60264341513 .8235871798-2.8773394543$

C $-0.05049253282 .1894985689-3.5426211001$ 

C $1.82341958824 .1494030233-4.2129243918$
H $2.1623343664 .3395175678-2.0998637333$
C $0.17125658432 .5185669157-4.878879134$
H - $0.77985157921 .4254683774-3.2922848335$
C $1.10692593673 .4986307988-5.2221947222$
H $2.55299703934 .9138706286-4.4686751437$
H - $0.38686725242 .0046575724-5.6580200842$
H $1.27664286823 .7525531614-6.265194616$

S8.3.2 - (5b-Pr)

44

C $-1.5547301167-2.3041776221-1.4285177692$

C $-0.7605171608-1.219488219-1.0606873283$

C $-1.3436850314-0.1683084223-0.2733876932$

C $-2.6939378572-0.35715171620 .1180438032$

C $-3.4785263909-1.4721416046-0.2548355909$

C $-2.9126354493-2.4465783964-1.0442504666$

H - $1.1156585817-3.0893899516-2.0386392053$

C -0.71711042641 .05126745430 .1586971775$

C -3.42085026690 .55063452950 .9214097719$

H -3.4726298916-3.3198822201-1.3695136497

C - 2.79254840771 .69346240951 .3581229333

C -1.44786336431 .92210691470 .966375157$

H -3.29847791352.42217224131.9869084784

H - 0.96467465322 .8308444721 .3173650862

P $1.025067231 .5036041679-0.2686316607$

P $0.9983315089-1.1240157792-1.6052383944$

C $0.7597988262 .4480159085-1.8951255431$

H $0.09822258121 .8398040281-2.5251149035$

C $2.11587710022 .59961487-2.5993090028$

H $2.00048716993 .1474145668-3.5437662821$

H $2.82553866793 .1598782798-1.9770574511$

H $2.55985335111 .6242849125-2.8255394665$

C $0.09384342143 .8138356431-1.6637559298$

H - $0.88847266763 .7126061095-1.1911041569$

H $0.71398641284 .4559805379-1.0258767179$

H -0.0471419099 4.3340349767-2.6210577728

C $-4.8695477367-1.32682103540 .3347189584$

H -5.1224118944 - 2.17624480970.9807012963

H -5.6340555869-1.2979551535-0.4512884762

C -4.82488389990 .01634884021 .139265926$

H -5.02866847757-0.1487388619 2.2043386775 
H -5.58471538570 .72340475490 .7851002632$

C $1.9547736719-2.0071776617-0.2826718125$

C $3.2426900717-1.54045318570 .0161407201$

C $1.4696950215-3.13577785620 .3941920235$

C $4.0365968316-2.19612559290 .960193482$

H $3.62202721-0.6549964329-0.4864355165$

C $2.2593230909-3.78711743831 .3423090109$

H $0.467467542-3.5006365530 .1873113999$

C $3.5458429954-3.32039373681 .6248783613$

H $5.0334630515-1.82301914391 .179926337$

H $1.8702333123-4.65940806941 .861533042$

H $4.159780815-3.82901359792 .3636493928$

H $0.9134401855-2.2143645565-2.5287675084$

\section{S8.3.3 - TS1}

53

(TS for Pr dissociation from (5b-H)·, see Figure 5)

C - $2.32752144512 .2787148653-0.7335728897$

C $-1.12628133071 .6024428316-0.5452649415$

C $-1.16515208660 .2555142164-0.0893827416$

C $-2.4252309897-0.3202991380 .1537285975$

C $-3.64065671850 .3655329895-0.043545846$

C $-3.59188621111 .6691544248-0.495293522$

H - $-2.30956002593 .3115441405-1.0729536273$

C $-0.0018766698-0.54239984840 .1240467304$

C $-2.5973627983-1.64309117160 .6094015956$

H - $4.4974098032 .2467907042-0.6652012466$

C $-1.4705647853-2.40968947130 .8185042419$

C $-0.1836642044-1.85149301920 .5635823213$

H - $1.5372641222-3.43779118021 .1666110285$

H $0.6802813708-2.48919357890 .7195384734$

P $1.61578606020 .2727601237-0.280598242$

P $0.55709044182 .3252983754-0.7353326237$

C 2.26237046040 .74651354351 .4563617225

H 1.44530035251 .29034576861 .9468983755

C $2.9515350903-1.8469027601-0.8474709011$

H $2.7847344157-2.44643783370 .0491183528$

C 3.46076929171 .69640817351 .3114006973

H 3.81195706982 .01627002262 .3010397776

H 4.29991689351 .20847921450 .8021637193

H 3.19654774572 .596384640 .7461287587

C $2.6127123633-0.46669447782 .3269381314$ 
H $1.7588148325-1.13982897072 .4515346039$

H $3.4462761139-1.04082107731 .9082536236$

H $2.9157825611-0.12850353063 .3265518821$

C $4.3654537629-1.3637737845-1.0265504761$

H $4.8063163-1.0050590131-0.0898234323$

H $5.0161374954-2.1729655382-1.4016895481$

H $4.4180933615-0.5482324961-1.7590701716$

C $2.2430152313-2.3347611755-2.0819693821$

H $2.7429571607-3.2262968871-2.4992349683$

H $1.2019825704-2.6080938663-1.8806545565$

H 2.2451406629-1.5700285186-2.8690517357

C $-4.7837293703-0.57142281050 .3148138068$

H - $5.4528812203-0.7346423026-0.5388528241$

H -5.4049147906 -0.1601378238 1.119735673

C $-4.086675898-1.91022403480 .7576644798$

H -4.4092765027-2.75292149560.1342745487

H -4.3475267599-2.1722362062 1.7903303817

C $0.76451503252 .4287330608-2.5715214547$

C $0.05464267371 .6445913573-3.4961530597$

C $1.70717408853 .348975887-3.0587566293$

C $0.28234617381 .7793695554-4.8660224362$

H - $0.68342017860 .9303789344-3.1428370376$

C $1.94101621023 .4788650352-4.4290055584$

H $2.25636402123 .9738751819-2.3580837045$

C $1.22720354842 .6949203058-5.3369686847$

H $-0.27725471451 .1658325398-5.5679076019$

H $2.6744150584 .1973517213-4.7863355478$

H $1.4025108512 .798367898-6.4046430525$

S8.3.4 - TS2

64

(TS for $\mathrm{H}$ abstraction from $\mathbf{5 b}$ by $\mathrm{Pr}$., see Figure 5 )

C -1.085902213 2.2849571618 1.3418537941

C -0.61073913251 .03514364780 .9417196949$

C -1.46827929020 .19906161150 .1304639429$

C $-2.76232963130 .7302932779-0.1419612287$

C -3.21788453191 .99768197190 .2921150854$

C - 2.37149360752 .78863849951 .0292073245

H - 0.43600126732 .91373159571 .9409710509

C - $1.1888055106-1.0971188254-0.4453507138$

C $-3.76038438560 .0630344413-0.8905932012$

H -2.6642547943 3.77462748711 .3819385245 
C -3.4817825596-1.1795837396-1.4036638187

C $-2.1966373576-1.7279788564-1.1726839062$

H - $4.2102252285-1.7401785856-1.9846791386$

H - $1.9943575119-2.6989207295-1.6124594585$

P $0.5048507838-1.8621093382-0.3064673425$

P 1.01184176140 .47245604471 .6466964403

C $0.2073110897-3.1074638811 .105929707$

H - $0.3326659977-2.49176289581 .8368325776$

C $0.6302674409-2.9119422258-1.8816494871$

H - $0.2097475958-3.6144771658-1.9586220488$

C $1.5350889789-3.52220787861 .7618663756$

H $1.3414355993-4.12858932532 .6567300665$

H $2.1582051798-4.12259538581 .090073869$

H $2.1132033704-2.64414423782 .0661313745$

C - $0.6685563229-4.32109768060 .7707154605$

H - $1.6393886898-4.02273792280 .3620146302$

H - $0.1841002579-4.98792722820 .0475221456$

H -0.8593061109-4.9112655771 1.6771539018

C $1.9308592971-3.7345316647-1.85227043$

H $1.9300533643-4.4891525782-1.059740859$

H $2.0634260747-4.2604608619-2.8067251007$

H $2.8053619624-3.0898416462-1.7033845636$

C $0.6229334243-1.9890816081-3.1122332844$

H $0.7446546884-2.5799928457-4.0295324232$

H -0.3081939325 - $1.4211153557-3.1985659174$

H $1.45054355-1.2710039687-3.0652931571$

C $-4.63774549492 .2265878466-0.1890249895$

H - $4.70333827483 .1151503657-0.8288345508$

H -5.3224960027 2.39919503070.6502281769

C $-5.00484004110 .925715849-0.9740194262$

H -5.272656087 1.1440528074-2.0149417704

H $-5.87154729070 .4194587661-0.531593313$

C 2.29137991381 .22220255050 .5430789378

C 3.63833016071 .00122445260 .8806654569

C $2.00803742971 .9718763535-0.6081711102$

C 4.66930702571 .53038025140 .1041412332

H 3.88189865080 .40189917511 .7559203907

C $3.04006974852 .4904204033-1.393731518$

H $0.97546536432 .1544707421-0.8901892247$

C $4.37320508682 .2764411997-1.0395044671$

H 5.70406782991 .35137279570 .3860364637

H $2.79959303073 .0667324638-2.2840011457$ 
H $5.17487407992 .6830464289-1.650523553$

H 1.11115489871 .54135761642 .7333637158

C 1.42547764622 .64846852094 .0208991601

H 0.38601153672 .81277494274 .3115279777

C 2.21761110421 .76130053774 .9386590245

H 2.49068938262 .28452036655 .8715608408

H 1.65638882710 .86381833865 .2229571573

H 3.15753292451 .4396439734 .4711565195

C 2.1180201263 .81339599293 .3719519009

H 1.47816987254 .31809479082 .6388535433

H 2.41520199334 .57191357284 .1178454722

H 3.03283333273 .49806733492 .8552038612 


\section{S9 - Notes and References}

(1) Wawrzyniak, P.; Fuller, A. L.; Slawin, A. M. Z.; Kilian, P. Inorg. Chem. 2009, 48, 2500.

(2) Kilian, P.; Philp, D.; Slawin, A. M. Z.; Woollins, J. D. Eur. J. Inorg. Chem. 2003, 249.

(3) Ray, M. J.; Slawin, A. M. Z.; Bühl, M.; Kilian, P. Organometallics 2013, 32, 3481.

(4) Taylor, L. J.; Surgenor, B. A.; Wawrzyniak, P.; Ray, M. J.; Cordes, D. B.; Slawin, A. M. Z.; Kilian, P. Dalton Trans. 2016, 45, 1976.

(5) When originally synthesised, $\mathbf{5 b}$ was obtained as an oil of limited purity. We report here an improved synthesis that yields $\mathbf{5} \mathbf{b}$ as a crystalline solid.

(6) Reference chemical shift of $p$-xylene methyl group determined by ${ }^{1} \mathrm{H}$ NMR spectroscopy of xylenes in $d_{8}$-toluene.

(7) Sheldrick, G. M. Acta Crystallogr., Sect. A 2008, A64, 112.

(8) Thomas, I. R.; Bruno, I. J.; Cole, J. C.; Macrae, C. F.; Pidcock, E.; Wood, P. A. J. Appl. Cryst. 2010, 43, 362.

(9) Bondi, A. J. Phys. Chem. 1964, 68, 441.

(10) Karaghiosoff, K. In Encyclopedia of Nuclear Magnetic Resonance; Grant, D. M., Harris, R. K., Eds.; Wiley: Chichester, U.K., Vol. 6, 1996; p 3612.

(11) Fulmer, G. R.; Miller, A. J. M.; Sherden, N. H.; Gottlieb, H. E.; Nudelman, A.; Stoltz, B. M.; Bercaw, J. E.; Goldberg, K. I. Organometallics 2010, 29, 2176.

(12) Zhang, Y.-H.; Mao, X.-A. Phosphorus Sulfur Silicon Relat. Elem. 2002, 177, 2409.

(13) Stevens, M. P. Polymer Chemistry: An Introduction; Oxford University Press USA: New York, 1999; pp 176-186.

(14) Marque, S.; Tordo, P. In Top. Curr. Chem.; Majoral, J. P., Ed.; Springer: Berlin, Germany, 2005; Vol. 250, pp 52-57.

(15) Leca, D.; Fensterbank, L.; Lacôte, E.; Malacria, M. Chem. Soc. Rev. 2005, 34, 858.

(16) Bullock, J. P.; Bond, A. M.; Boeré, R. T.; Gietz, T. M.; Roemmele, T. L.; Seagrave, S. D.; Masuda, J. D.; Parvez, M. J. Am. Chem. Soc. 2013, 135, 11205.

(17) Fleming, J. T.; Higham, L. J. Coord. Chem. Rev. 2015, 297-298, 127.

(18) Yasui, S.; Tojo, S.; Majima, T. J. Org. Chem. 2005, 70, 1276.

(19) Liu, L. (Leo); Cao, L. L.; Shao, Y.; Ménard, G.; Stephan, D. W. Chem 2017, 3, 259.

(20) Becke, A. D. J. Chem. Phys. 1993, 98, 5648.

(21) Lee, C.; Yang, W.; Parr, R. G. Phys. Rev. B 1988, 37, 785.

(22) Ciofini, I.; Daul, C. A. Coord. Chem. Rev. 2003, 238-239, 187.

(23) Zhao, Y.; Truhlar, D. G. Theor. Chem. Acc. 2008, 120, 215.

(24) Chalmers, B. A.; Bühl, M.; Arachchige, K. S. A.; Slawin, A. M. Z.; Kilian, P. J. Am. Chem. Soc. 2014, $136,6247$.

(25) Mennucci, B.; Tomasi, J. J. Chem. Phys. 1997, 106, 5151.

(26) Tomasi, J.; Mennucci, B.; Cancès, E. J. Mol. Struct. (Theochem.) 1999, 464, 211.

(27) Tomasi, J.; Mennucci, B.; Cammi, R. Chem. Rev. 2005, 105, 2999.

(28) Frisch, M. J.; Trucks, G. W.; Schlegel, H. B.; Scuseria, G. E.; Robb, M. A.; Cheeseman, J. R.; Scalmani, G.; Barone, V.; Mennucci, B.; Petersson, G. A.; Nakatsuji, H.; Caricato, M.; Li, X.; Hratchian, H. P.; Izmaylov, A. F.; Bloino, J.; Zheng, G.; Sonnenberg, J. L.; Hada, M.; Ehara, M.; Toyota, K.; Fukuda, R.; Hasegawa, J.; Ishida, M.; Nakajima, T.; Honda, Y.; Kitao, O.; Nakai, H.; Vreven, T.; Montgomery, J.; Peralta, J. A. J. E.; Ogliaro, F.; Bearpark, M.; Heyd, J. J.; Brothers, E.; Kudin, K. N.; Staroverov, V. N.; Kobayashi, R.; Normand, J.; Raghavachari, K.; Rendell, A.; Burant, J. C.; Iyengar, S. S.; Tomasi, J.; Cossi, M.; Rega, N.; Millam, N. J.; Klene, M.; Knox, J. E.; Cross, J. B.; Bakken, V.; Adamo, C.; Jaramillo, J.; Gomperts, R.; Stratmann, R. E.; Yazyev, O.; Austin, A. J.; Cammi, R.; Pomelli, C.; Ochterski, J. W.; Martin, R. L.; Morokuma, K.; Zakrzewski, V. G.; Voth, G. A.; Salvador, P.; Dannenberg, J. J.; Dapprich, 
S.; Daniels, A. D.; Farkas, Ö.; Foresman, J. B.; Ortiz, J. V.; Cioslowski, J.; Fox, D. J. Gaussian 09; Wallingford CT, 2009. 